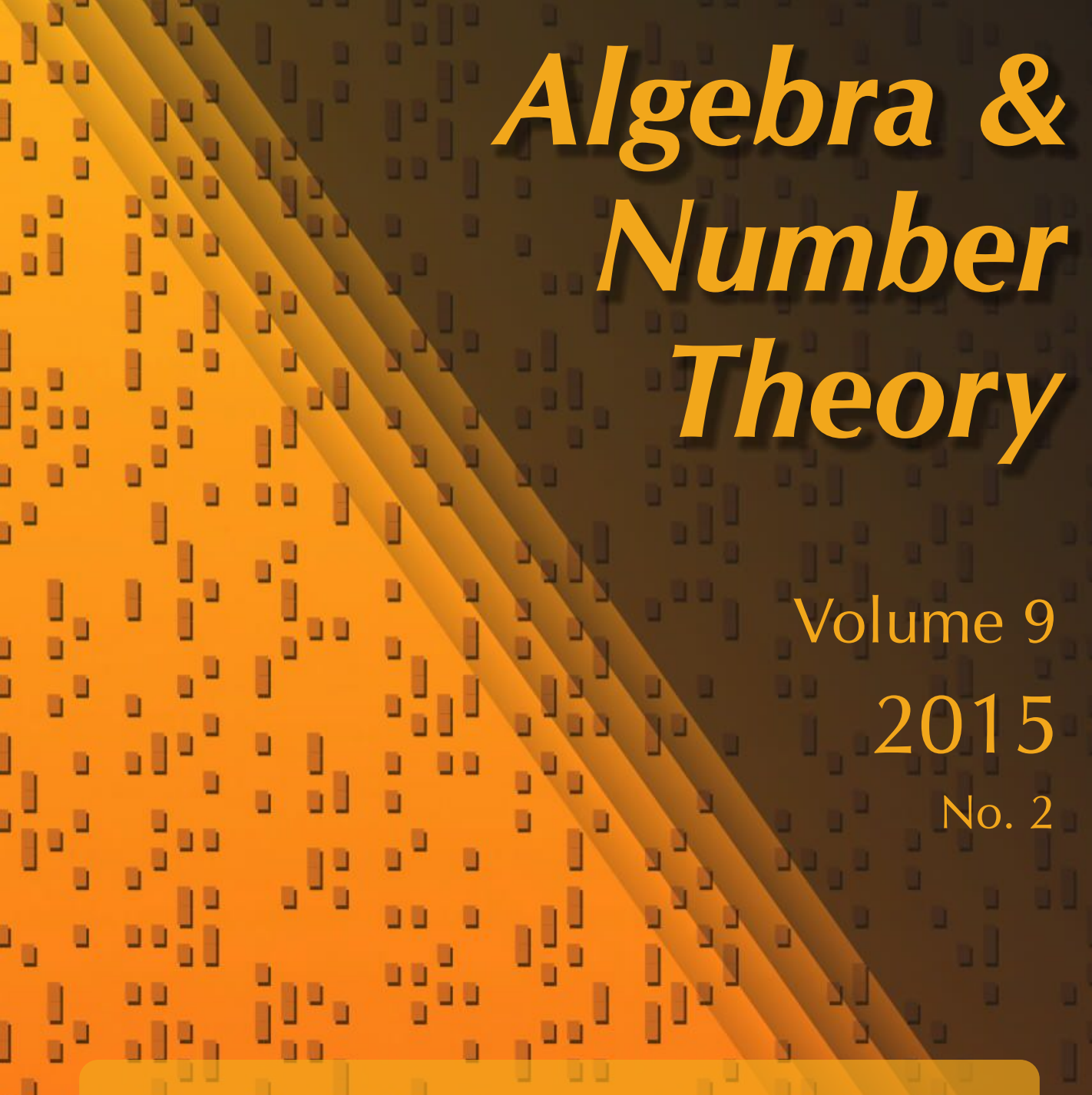




\title{
Noncommutative Hilbert modular symbols
}

\author{
Ivan Horozov
}

\begin{abstract}
The main goal of this paper is to construct noncommutative Hilbert modular symbols. However, we also construct commutative Hilbert modular symbols. Both the commutative and the noncommutative Hilbert modular symbols are generalizations of Manin's classical and noncommutative modular symbols. We prove that many cases of (non)commutative Hilbert modular symbols are periods in the Kontsevich-Zagier sense. Hecke operators act naturally on them.

Manin defined the noncommutative modular symbol in terms of iterated path integrals. In order to define noncommutative Hilbert modular symbols, we use a generalization of iterated path integrals to higher dimensions, which we call iterated integrals on membranes. Manin examined similarities between noncommutative modular symbol and multiple zeta values in terms of both infinite series and of iterated path integrals. Here we examine similarities in the formulas for noncommutative Hilbert modular symbol and multiple Dedekind zeta values, recently defined by the current author, in terms of both infinite series and iterated integrals on membranes.
\end{abstract}

1. Introduction

2. Manin's noncommutative modular symbol

3. Iterated integrals on membranes

4. Hilbert modular symbols

Acknowledgements

367

References

\section{Introduction}

Classical elliptic modular symbols were introduced by Birch [1971] and Manin [1972] in connection with the Birch-Swinnerton-Dyer conjecture for certain congruence subgroups of $\mathrm{SL}_{2}(\mathbb{Z})$. We recall that a modular symbol $\{p, q\}$ is associated to a pair of cusp points $p, q \in \mathbb{P}^{1}(\mathbb{Q})$ on the completed upper half-plane $\mathbb{P}^{1} \cup \mathbb{P}^{1}(\mathbb{Q})$. One can think of the modular symbol $\{p, q\}$ as a homology class of the geodesic

MSC2010: primary 11F41; secondary 11F67, $11 \mathrm{M} 32$.

Keywords: modular symbols, Hilbert modular groups, Hilbert modular surfaces, iterated integrals. 
connecting $p$ and $q$, in $H_{1}\left(X_{\Gamma}\right.$, \{cusps\}), where $X_{\Gamma}$ is the modular curve associated to a congruence subgroup of $\mathrm{SL}_{2}(\mathbb{Z})$. One can pair $\{p, q\}$ with a cusp form $f$ by

$$
\{p, q\} \times f \mapsto \int_{p}^{q} f d z
$$

If $f$ is a cusp form of weight 2 then $f d z$ can be viewed as a cohomology class in $H^{1}\left(X_{\Gamma}\right)$. This gives a pairing between homology (Betti) and cohomology (de Rham) that leads to periods. Modular symbols are a useful tool for studying $L$ functions and in the computation of cohomology groups. For a review of such topics, one can consult [Manin 2009].

Their theory was developed in [Manin 1972; Drinfeld 1973; Shokurov 1980; Mazur 1973]. Later the theory was extended to higher ranks in [Ash and Rudolph 1979; Ash and Borel 1990; Gunnells 2000b].

Elliptic modular symbols are important tool in the study of modular forms. They are particularly useful in computations with modular forms. J. Cremona [1997] designed algorithms for computations with elliptic curves, based on modular symbols ("modular symbol algorithms"). Some of the applications include the computation of homology and cohomology. Also, the study of special values of $L$-functions became a vast area of applications of classical modular symbols; see [Mazur and Swinnerton-Dyer 1974; Kazhdan et al. 2000].

Later, W. Stein also contributed to the difficult area of computations with modular forms. See his excellent book [Stein 2007], which contains both theory and computational methods. For higher-rank groups, one can consult the appendix of this book, by P. Gunnells.

Manin's noncommutative modular symbol [2006] is a generalization of both the classical modular symbol and of multiple zeta values in terms of Chen's iterated integral theory in the holomorphic setting. Manin showed that the noncommutative modular symbol is a noncommutative 1-cocycle. He also showed that each of the iterated integrals on Hecke eigenforms that enter in the noncommutative modular symbol are periods.

The main goal of this paper is to construct noncommutative Hilbert modular symbols. However, we also construct an analog of the classical modular symbol for Hilbert modular varieties. Both symbols are generalizations of the corresponding constructions by Manin.

We compute explicit integrals in terms of the noncommutative Hilbert modular symbol of type $\boldsymbol{b}$, and present similar formulas for the recently defined multiple Dedekind zeta values (see [Horozov 2014b]). We prove that the iterated integrals on membranes that enter in the noncommutative modular symbol of type $c$ are periods. We also give some explicit and some categorical arguments in support of a 
conjecture that a certain type of noncommutative Hilbert modular symbol satisfies a noncommutative 2-cocycle condition.

Before describing our results, let us recall the noncommutative modular symbol of Manin [2006]. Let $\nabla=d-\sum_{i=1}^{m} X_{i} f_{i} d z$ be a connection on the upper halfplane, where $f_{1}, \ldots, f_{m}$ are cusp forms and $X_{1}, \ldots, X_{m}$ are formal variables. One can think of $X_{1}, \ldots, X_{m}$ as constant square matrices of the same size.

Let $J_{a}^{b}$ be the parallel transport to the point $b$ of the identity matrix 1 at the point $a$. Alternatively, $J_{b}^{a}$ can be written as a generating series of iterated path integrals of the forms $f_{1} d z, \ldots, f_{m} d z$, (see [Chen 1977] and [Manin 2006]), namely,

$$
J_{a}^{b}=1+\sum_{i=1}^{m} X_{i} \int_{a}^{b} f_{i} d z+\sum_{i, j=1}^{m} X_{i} X_{j} \int_{a}^{b} f_{i} d z \cdot f_{j} d z+\cdots
$$

Then $J_{a}^{b} J_{b}^{c}=J_{a}^{c}$. This property leads to the 1-cocycle $c_{a}^{1}(\gamma)=J_{\gamma a}^{a}$ for $\gamma \in \mathrm{SL}_{2}(\mathbb{Z})$, which is the noncommutative modular symbol (see [Manin 2006] and Section 2 of this paper). If $f_{1}, \ldots, f_{m}$ are normalized cusp Hecke eigenforms, then each iterated integral appearing in the generating series $J_{a}^{b}$ is a period. In this paper we introduce both commutative and noncommutative modular symbols for Hilbert modular surfaces. As it turned out we need some new tools, in comparison to the classical modular symbols. In particular, for the noncommutative Hilbert modular symbol we need iterated integrals in dimension higher than one. We introduce them and study their properties in the special case of Hilbert modular surfaces for the Hilbert modular group $\mathrm{SL}_{2}\left(\mathrm{O}_{K}\right)$. For the Hilbert modular group, one may consult [Bruinier et al. 2008] and [Freitag 1990]. In the case of Hilbert modular surface, it is not possible to repeat Manin's constructions for the noncommutative modular symbols, since the integration domain is two-dimensional over the complex numbers. Instead, we develop a new approach (Section 3), which we call iterated integrals on membranes. This is a higher-dimensional analogue of iterated path integrals. In Section 4G, we explore similar relations between noncommutative Hilbert modular symbols and multiple Dedekind zeta values (see [Horozov 2014b]).

In Section 4, we associate modular symbols for $\mathrm{SL}_{2}\left(\mathscr{O}_{K}\right)$ to geodesic triangles and geodesic diangles (2-cells whose boundaries have two vertices and two edges, which are geodesics). We are going to explain how the geodesic triangles and the geodesic diangles are constructed. Consider four cusp points in $\mathbb{T}^{2} \cup \mathbb{P}^{1}(K)$. We can map any three of them to 0,1 and $\infty$ with a linear fractional transformation $\gamma \in \mathrm{GL}_{2}(K)$. There is a diagonal map $\mathbb{H}^{1} \rightarrow \mathbb{H}^{2}$, whose image $\Delta$ contains 0,1 and $\infty$. We can take a pullback of $\Delta$ with respect to the map $\gamma$ in order to obtain a holomorphic (or antiholomorphic) curve that passes through the given three points. If $\operatorname{det} \gamma$ is totally positive or totally negative then $\gamma^{*} \Delta$ is a holomorphic curve in $\mathbb{U}^{2}$. If $\operatorname{det} \gamma$ is not totally positive or totally negative (that is, in one of the real embeddings 
it is positive and in the other it is negative) then $\gamma^{*} \Delta$ is antiholomorphic. This means that it is holomorphic in $\mathbb{Q}^{2}$ if we conjugate the complex structure in one of the copies of $\mathbb{M}^{1}$. The same type of change of the complex structure is considered in [Freitag 1990].

On each holomorphic (or antiholomorphic) curve $\gamma^{*} \Delta$, there is a unique geodesic triangle connecting the three given points. However, if we take two of the points, we see that they belong to two geodesic triangles. Thus they belong to two holomorphic, (antiholomorphic) curves. Therefore, there are two geodesic connecting the two points - each lying on different holomorphic (antiholomorphic) curves, as faces of the corresponding geodesic triangles defining the curves. There are two pairings that we consider: the first is an integral of a cusp form over a geodesic triangle and the second is an integral of a cusp form over a geodesic diangle. If we integrate a holomorphic 2-form coming from a cusp form over a geodesic triangle, we obtain 0 , if the triangle lies on an holomorphic curve. Thus the only nonzero pairings come from integration of a cusp form over a diangle or over a triangle lying on an antiholomorphic curve.

Now let us look again at the four cusp points together with the geodesics that we have just described. We obtain four geodesic triangles, corresponding to each triple of points among the four points, and six diangles, corresponding to the six "edges" of a tetrahedron with vertices the four given points. Thus, we obtain a "tetrahedron" with thickened edges. We will use tetrahedrons with thickened edges as an intuition for a noncommutative 2-cocycle relation (see Conjecture 4.15) for the noncommutative Hilbert modular symbol, which is an analogue of Manin's noncommutative 1-cocycle relation for the noncommutative modular symbol.

Usually, the four vertices are treated as a tetrahedron and a 2-cocycle is functional on the faces, considered as 2-chains. The boundary is defined as a sum of the 2cocycles on each of the faces (which are triangles). The boundary of the tetrahedron gives a boundary relation of a 2-cocycle.

In our case the analogue of a 2-cocycle is a functional on diangles and on triangles. And the boundary map is a sum over the faces of the thickened tetrahedron. Thus, the faces of the thickened tetrahedron are four triangles and six diangles, corresponding to the six edges of a tetrahedron.

We show that the geodesics on the boundary of a diangle or of a geodesic triangle lie on a holomorphic curves $\gamma^{*} \Delta$ for various elements $\gamma$ with totally positive or totally negative determinant. This implies that when we take the quotient by a Hilbert modular group the holomorphic curve $\gamma^{*} \Delta$ becomes a Hirzebruch-Zagier divisor [Hirzebruch and Zagier 1976]. Then we prove that the commutative Hilbert modular symbols paired with a cusp forms of weight $(2,2)$ gives periods in the sense of [Kontsevich and Zagier 2001]. 
In order to construct a noncommutative Hilbert modular symbol, first we define a suitable generalization of iterated path integrals, which we call iterated integrals on membranes (see Section 3). We choose the word "membrane" since such integrals are invariant under suitable variation of the domain of integration.

There is a topological reason for considering a noncommutative Hilbert modular symbol as opposed to only a commutative one. Let us first make such a comparison for the case of $\mathrm{SL}_{2}(\mathbb{Z})$. The commutative modular symbol captures $H_{1}\left(X_{\Gamma}\right)$, while the noncommutative symbol captures the rational homotopy type of the modular curve $X_{\Gamma}$. Now, let $\tilde{X}$ be a smooth Hilbert modular surface, by which we mean the minimal desingularization of the Borel-Baily compactification due to Hirzebruch. Then the rational fundamental group of a Hilbert modular surface vanishes: $\pi_{1}(\tilde{X})_{\mathbb{Q}}=0$ (see [Bruinier et al. 2008]). The noncommutative Hilbert modular symbol is an attempt to capture more from the rational homotopy type than $H_{2}(\tilde{X})$ captures.

For the convenience of the reader, we first define type $\boldsymbol{a}$ iterated integrals on membranes (Definition 3.3). They are simpler to define and more intuitive. However, they do not have enough properties. (For example, they do not have an integral shuffle relation.) Then we define type $\boldsymbol{b}$ iterated integrals on membranes (Definition 3.4), which involves two permutations. Type $\boldsymbol{b}$ has integral shuffle relation (Theorem 3.21(i)), and type $\boldsymbol{a}$ is a particular case of type $\boldsymbol{b}$.

We are mostly interested in iterated integrals of type $\boldsymbol{b}$. If there is no index specifying the type of iterated integral over membranes, we assume that it is of type $\boldsymbol{b}$.

Similarly to Manin's approach, we define a generating series of iterated integrals over membrane of type $\boldsymbol{b}$ over $U$, which we denote by $J(U)$. We also define a shuffle product of generating series of iterated integrals over membranes of type $\boldsymbol{b}$ (see Theorem 3.21(iii)),

$$
\phi\left(J\left(U_{1}\right) \times_{\mathrm{Sh}} J\left(U_{2}\right)\right)=J\left(U_{1} \cup U_{2}\right),
$$

for $U_{1}, U_{2}$ disjoint 2-dimensional manifolds with corners contained in $\mathbb{T}^{2} \cup \mathbb{P}^{1}(K)$ (see [Borel and Serre 1973]). This shuffle product generalizes the composition of generating series of iterated path integrals, namely, $J_{a}^{b} J_{b}^{c}=J_{a}^{c}$, to dimension 2 . Note that a similar definition is also possible in higher dimensions. Also, $J(U)$ is invariant under homotopy. This allows us to consider cocycles and coboundaries, where the relations use homotopy invariance and values at different cells can be composed via the shuffle product.

We define noncommutative Hilbert modular symbols, which we call $c^{1}$ and $c^{2}$; $c^{1}$ is the functional $J$ on certain geodesic diangles and $c^{2}$ is the functional $J$ on geodesic triangles. Conjecturally, $c^{1}$ is a 1-cocycle such that if we change the base point of $c^{1}$ then $c^{1}$ is modified by a coboundary. Also, conjecturally, $c^{2}$ is a 2-cocycle up to finitely many multiples of different values of $c^{1}$. Also, if we 
change the base point of $c^{2}$ then $c^{2}$ is modified by a coboundary up to a finitely many multiples of different values of $c^{1}$. In Section $4 \mathrm{E}$ we give explicit formulas in support of the interpretation of the noncommutative symbols as cocycles.

In Section 4F, we give a categorical construction, which might help to prove that the noncommutative symbols are cocycles.

In Section 4G, we define multiple $L$-values associated to cusp forms, and we compare them to multiple Dedekind zeta values (see [Horozov 2014b]).

We also briefly recall the Riemann zeta values and multiple zeta values (MZVs). The Riemann zeta values are defined as

$$
\zeta(k)=\sum_{n>0} \frac{1}{n^{k}},
$$

where $n$ is an integer. MZVs are defined as

$$
\zeta\left(k_{1}, \ldots, k_{m}\right)=\sum_{0<n_{1}<\cdots<n_{m}} \frac{1}{n_{1}^{k_{1}} \cdots n_{m}^{k_{m}}},
$$

where $n_{1}, \ldots, n_{m}$ are integers. The above MZV is of depth $m$. Riemann zeta values $\zeta(k)$ and MZV $\zeta\left(k_{1}, \ldots, k_{m}\right)$ were defined by Euler [1748] for $m=1,2$.

The common feature of MZVs and the noncommutative modular symbol is that they both can be written as iterated path integrals (see [Goncharov 2001a; 2001b]). Moreover, Manin's noncommutative modular symbol resembles the generating series of MZV, which is the Drinfeld associator. Let us recall that the Drinfeld associator is a generating series of iterated integrals of the type $J_{a}^{b}$ associated to the connection

$$
\nabla=d-A \frac{d x}{x}-B \frac{d x}{1-x}
$$

on $Y_{\Gamma(2)}=\mathbb{P}^{1}-\{0,1, \infty\}$. One can think of $Y_{\Gamma(2)}$ as the modular curve associated to the congruence subgroup $\Gamma(2)$ of $\mathrm{SL}_{2}(\mathbb{Z})$. Then the differential forms $d x / x$ and $d x /(1-x)$ are Eisenstein series of weight 2 on the modular curve $Y_{\Gamma(2)}$.

Relations between MZV and modular forms have been examined by many authors. For example, Goncharov [2001b; 2001c] considered a mysterious relation between MZV (multiple zeta values) of given weight and depth 3 and the cohomology of $\mathrm{GL}_{3}(\mathbb{Z})$, which is closely related to the cohomology of $\mathrm{SL}_{3}(\mathbb{Z})$. In the pursuit of such a relation in depth 4 , Goncharov suggested and the current author computed the group cohomology of $\mathrm{GL}_{4}(\mathbb{Z})$ with coefficients in a family of representations [Horozov 2014a]. Another relation between modular forms and MZV is presented in [Gangl et al. 2006].

Similarly to Manin's approach, we explore relations between the noncommutative Hilbert modular symbols and multiple Dedekind zeta values (see [Horozov 2014b]). Let us recall multiple Dedekind zeta values. Let each of $C_{1}, \ldots, C_{m}$ be a suitable 
subset of the ring of integers $O_{K}$ of a number field $K$. We call each of $C_{1}, \ldots, C_{m}$ a cone. Then multiple Dedekind zeta values are defined as

$$
\zeta_{K ; C_{1}, \ldots, C_{m}}\left(k_{1}, \ldots, k_{m}\right)=\sum_{\alpha_{i} \in C_{i}} \frac{1}{N\left(\alpha_{1}\right)^{k_{1}} N\left(\alpha_{1}+\alpha_{2}\right)^{k_{2}} \cdots N\left(\alpha_{1}+\cdots+\alpha_{m}\right)^{k_{m}}} .
$$

The connection between noncommutative Hilbert modular symbols and multiple Dedekind zeta values lies both in the similarities in the infinite sum formulas and in the definition in terms of iterated integrals on membranes (see [Horozov 2014b]).

We consider a noncommutative Hilbert modular symbol of type $\boldsymbol{b}$ over one diangle and compare it with (multiple) Dedekind zeta values with summation over one discrete cone [Horozov 2014b]. However, in this case the two series look very different. We obtain that the multiple $L$-values are noncommutative modular symbols defined as $J$ evaluated at an infinite union of diangles. We obtain that such $L$-values are very similar to the sum of multiple Dedekind zeta values, in the same way that the integrals in Manin's noncommutative modular symbol are similar to the multiple zeta values (MZV). Then the sum of the multiple Dedekind zeta values is over an infinite union of cones. The idea of considering cones originated in [Zagier 1976] and more generally in [Shintani 1976].

Classical or commutative modular symbols for $\mathrm{SL}_{3}(\mathbb{Z})$ and $\mathrm{SL}_{4}(\mathbb{Z})$ were constructed in [Ash and Borel 1990] and [Gunnells 2000a]. For $\mathrm{GL}_{2}\left(\mathrm{O}_{K}\right)$, where $K$ is a real quadratic field, Gunnells and Yasaki [2008] defined a modular symbol based on Voronoi decomposition of a fundamental domain, in order to compute the third cohomology group of $\mathrm{GL}_{2}\left(\mathrm{O}_{K}\right)$. (For the Hilbert modular group $\mathrm{SL}_{2}\left(\mathrm{O}_{K}\right)$ one may consult [Bruinier et al. 2008; Freitag 1990].) In contrast, here we use a geodesic triangulation of $\mathbb{M}^{2} / \mathrm{SL}_{2}\left(\mathrm{O}_{K}\right)$. We are interested mostly in 2-cells, whose boundaries are geodesics. One of the (commutative) symbols that we define here resembles combinatorially the symplectic modular symbol of [Gunnells 2000b]. However, the meanings of the two types of symbols and their approaches are different.

There are several different directions for further work on Hilbert modular symbols. First of all, the commutative Hilbert modular symbols behave well with respect to Hecke operators. It will be interesting to extend the Hecke operators to cases of higher equal weights $(k, k)$. To apply Hecke operators to Hilbert modular groups one either assumes a trivial narrow class group or one has to work with adeles. Another possible continuation of the current work is to extend commutative Hilbert modular symbols to the adelic setting. Then, one may try to extend these properties higher equal weight cusp forms and Hecke operators in the adelic setting — to the noncommutative Hilbert modular symbols. Hopefully, the abelian Hilbert modular symbol would lead to computational tools for cohomology of some Hilbert modular groups with coefficients in various representations. 
For the noncommutative Hilbert modular symbols we expect that some of the continuations would be establishing the 2-categorical framework that define nonabelian 2-cohomology sets. This work would also have applications to noncommutative reciprocity laws on algebraic surfaces. In dimension 1, we have a noncommutative reciprocity law as a reciprocity law for a generating series of iterated path integrals on a complex curve [Horozov 2011]. In dimension 2 we have proven both the Parshin reciprocity and a new reciprocity for a 4-function local symbols [Horozov 2014c] defined by the author, which are particular cases in the generating series. A 2-categorical second cohomology set would capture algebraically the generating series of iterated integrals on membranes needed for the general reciprocity on algebraic surfaces.

Finally, we expect that the (non)commutative Hilbert modular symbols would be useful for studying $L$-functions and multiple $L$-functions together with their special values.

\section{Manin's noncommutative modular symbol}

In this section we recall the definition and main properties of Manin's [2006] noncommutative modular symbol. In this paper, Manin uses iterated path integrals on a modular curve and on its universal cover - the upper half-plane. Our main constructions are parallel to some extent to Manin's approach, and for that reason we recall it below. However, instead of iterated path integrals we introduce a new tool - iterated integrals on membranes (see Section 3). Only this notion is adequate for studying noncommutative Hilbert modular symbols, by generalizing the iteration process to higher dimensions.

2A. Iterated path integrals. Here we recall iterated path integrals (see also [Parshin 1966; Chen 1977; Goncharov 2001a; Manin 2006]). In Section 3, we generalize them to iterated integrals over membranes.

Definition 2.1. Let $\omega_{1}, \ldots, \omega_{m}$ be $m$ holomorphic 1-forms on $\mathbb{M}^{1} \cup \mathbb{P}^{1}(\mathbb{Q})$, the upper half-plane together with the cusps. Let

$$
g:[0,1] \rightarrow \mathbb{M}^{1} \cup \mathbb{P}^{1}(\mathbb{Q}),
$$

be a piecewise smooth path. We define an iterated integral

$$
\int_{g} \omega_{1} \cdots \omega_{m}=\int \cdots \int_{0<t_{1}<t_{2}<\cdots<t_{m}<1} g^{*} \omega_{1}\left(t_{1}\right) \wedge \cdots \wedge g^{*} \omega_{m}\left(t_{m}\right) .
$$

Let $X_{1}, \ldots, X_{m}$ be formal variables. Consider the differential equation

$$
d F(\Omega)=F(\Omega)\left(X_{1} \omega_{1}+\cdots+X_{n} \omega_{m}\right)
$$


with values in the associative but noncommutative ring of formal power series in the noncommuting variables $X_{1}, \ldots, X_{m}$ over the ring of holomorphic functions on the upper half-plane. There is a unique solution with initial condition $F(\Omega)(g(0))=1$; that is, equal to 1 at the starting point $g(0)$. Then at the end of the path, that is, at the point $g(1), F(\Omega)$ has the value

$$
F_{g}(\Omega)=1+\sum_{i=1}^{m} X_{i} \int_{g} \omega_{i}+\sum_{i, j=1}^{m} X_{i} X_{j} \int_{g} \omega_{i} \omega_{j}+\sum_{i, j, k=1}^{m} X_{i} X_{j} X_{k} \int_{g} \omega_{i} \omega_{j} \omega_{k}+\cdots
$$

Using the solution (2) to (1), we prove the following theorem:

Theorem 2.2. Let $g_{1}$ and $g_{2}$ be two paths such that the end of $g_{1}$ (i.e., $\left.g_{1}(1)\right)$ is equal to the beginning of $g_{2}\left(i . e ., g_{2}(0)\right)$. Let $g_{1} g_{2}$ denote the concatenation of $g_{1}$ and $g_{2}$. Then

$$
F_{g_{1} g_{2}}(\Omega)=F_{g_{1}}(\Omega) F_{g_{2}}(\Omega)
$$

Proof. The left-hand side is the value of the solution of the linear first-order ordinary differential equation at the point $g_{2}(1)$. From the uniqueness of the solution, we have that the solution along $g_{2}$ gives the same result, when the initial condition at $g_{2}(0)$ is $F_{g_{1}}(\Omega)$. That result is $F_{g_{1}}(\Omega) F_{g_{2}}(\Omega)$.

The same result can be proven via product formula for iterated integrals. We need this alternative proof in order to generalize to higher dimensions.

Lemma 2.3 (product formula). Let $\omega_{1}, \ldots, \omega_{m}$ be holomorphic 1-forms on $\mathbb{C}$ and $g_{1}, g_{2}$ two paths such that the end of $g_{1}$ is the beginning of $g_{2}$, that is, $g_{1}(1)=g_{2}(0)$. As before we denote by $g_{1} g_{2}$ the concatenation of the paths $g_{1}$ and $g_{2}$. Then

$$
\int_{g_{1} g_{2}} \omega_{1} \cdots \omega_{m}=\sum_{i=0}^{m} \int_{g_{1}} \omega_{1} \cdots \omega_{i} \int_{g_{2}} \omega_{i+1} \cdots \omega_{m} .
$$

Proof. Let $g_{1}:[0,1] \rightarrow \mathbb{C}$ and let $g_{2}:[1,2] \rightarrow \mathbb{C}$. We consider the concatenation $g_{1} g_{2}$ to be a map $g_{1} g_{2}:[0,2] \rightarrow \mathbb{C}$ whose restriction to the interval $[0,1]$ gives the path $g_{1}$ and whose restriction to the interval [1, 2] gives $g_{2}$. From Definition 2.1, we have that

$$
\int_{g_{1} g_{2}} \omega_{1} \cdots \omega_{m}=\int \cdots \int_{0<t_{1}<\cdots<t_{m}<2}\left(g_{1} g_{2}\right)^{*} \omega_{1}\left(t_{1}\right) \wedge \cdots \wedge\left(g_{1} g_{2}\right)^{*} \omega_{m}\left(t_{m}\right) .
$$

In the domain of integration $0<t_{1}<\cdots<t_{m}<2$ insert the number 1. Geometrically, we cut the simplex $0<t_{1}<\cdots<t_{m}<2$ into a disjoint union of products of pairs of simplices such that $t_{k} \in[0,1]$ for $k \leq i$ and $t_{k} \in[1,2]$ for $k>i$. Thus, the union is over distinct values of $i$ for $i=0, \ldots, m$. And for each fixed $i$ the two simplices 
are $0<t_{1}<\cdots<t_{i}<1$ and $1<t_{i+1}<\cdots<t_{m}<2$. Then we have

$$
\begin{aligned}
\int_{g_{1} g_{2}} \omega_{1} \cdots \omega_{m}= & \sum_{i=0}^{n} \int \cdots \int_{\substack{0<t_{1}<\cdots<t_{i}<1 \\
1<t_{i+1}<\cdots<t_{m}<2}}\left(g_{1} g_{2}\right)^{*} \omega_{1}\left(t_{1}\right) \wedge \cdots \wedge\left(g_{1} g_{2}\right)^{*} \omega_{m}\left(t_{m}\right) \\
= & \sum_{i=0}^{n}\left(\int \cdots \int_{\substack{0<t_{1}<\cdots<t_{i}<1 \\
g_{1}}} g_{1}^{*} \omega_{1}\left(t_{1}\right) \wedge \cdots \wedge g_{1}^{*} \omega_{i}\left(t_{i}\right)\right) \\
& \times\left(\int \cdots \int_{1<t_{i+1}<\cdots<t_{m}<2} g_{2}^{*} \omega_{i+1}\left(t_{i+1}\right) \wedge \cdots \wedge g_{2}^{*} \omega_{m}\left(t_{m}\right)\right) \\
= & \sum_{i=0}^{m} \int_{g_{1}} \omega_{1} \cdots \omega_{i} \int_{g_{2}} \omega_{i+1} \cdots \omega_{m} .
\end{aligned}
$$

Definition 2.4. The set of all shuffles $\operatorname{sh}(i, j)$ is a subset of all permutations $\sigma$ of the set $\{1,2, \ldots, i+j\}$ such that

$$
\sigma(1)<\cdots<\sigma(i)
$$

and

$$
\sigma(i+1)<\cdots<\sigma(i+j) .
$$

Such a permutation $\sigma$ is called a shuffle.

Lemma 2.5 (shuffle relation). Let $\omega_{1}, \ldots, \omega_{m}$ be holomorphic 1 -forms on $\mathbb{C}$ and let $g$ be a path. Then

$$
\int_{g} \omega_{1} \cdots \omega_{i} \int_{g} \omega_{i+1} \cdots \omega_{m}=\sum_{\sigma \in \operatorname{sh}(i, m-i)} \int_{g} \omega_{\rho(1)} \cdots \omega_{\rho(m)},
$$

where $\operatorname{sh}(i, j)$ is the set of shuffles from Definition 2.4.

2B. Manin's noncommutative modular symbol. Now let $g$ be a geodesic connecting two cusps $a$ and $b$ in the completed upper half-plane $\mathbb{Q}^{1} \cup \mathbb{P}^{1}(\mathbb{Q})$. Let $\Omega=\left\{f_{1} d z, \ldots, f_{m} d z\right\}$ be a finite set of holomorphic forms with respect to a congruence subgroup $\Gamma$ of $\mathrm{SL}_{2}(\mathbb{Z})$ such that $f_{1}, \ldots, f_{m}$ are cusp forms of weight 2 . Let

$$
J_{a}^{b}=F_{g}(\Omega) .
$$

As a reformulation of Theorem 2.2, we obtain:

Lemma 2.6.

$$
J_{a}^{b} J_{b}^{c}=J_{a}^{c} .
$$

The following is a direct consequence:

Corollary 2.7.

$$
J_{b}^{a}=\left(J_{a}^{b}\right)^{-1} .
$$


Now we are ready to define Manin's noncommutative modular symbol. Note that there is a natural action of $\Gamma$ on $J_{a}^{b}$. If $\gamma \in \Gamma$ then $\gamma J_{a}^{b}$ is defined as $J_{\gamma a}^{\gamma b}$. If $f_{1}, \ldots, f_{m}$ are cusp forms of weight 2 , then $\omega_{1}=f_{1} d z, \ldots, \omega_{m}=f_{m} d z$ are forms of weight 0 , that is, they are invariant forms with respect to the group $\Gamma$. Then

$$
\gamma J_{a}^{b}=F_{\gamma g}\left(\omega_{1}, \ldots, \omega_{m}\right)=F_{g}\left(g^{*} \omega_{1}, \ldots, g^{*} \omega_{m}\right)=F_{g}\left(\omega_{1}, \ldots, \omega_{m}\right)=J_{a}^{b} .
$$

Let $\Pi$ be the subgroup of invertible elements of $\mathbb{C}\left\langle\left\langle X_{1}, \ldots, X_{m}\right\rangle\right\rangle$ with constant term 1 . We extend the action of $\Gamma$ on $J_{a}^{b}$ to a trivial action of $\Gamma$ on $\Pi$.

Following Manin, we present the key theorem and definition for the noncommutative modular symbol:

Theorem 2.8. Let

$$
c_{a}^{1}(\gamma)=J_{\gamma a}^{a} .
$$

Then $c_{a}^{1}$ represent a cohomology class in $H^{1}(\Gamma, \Pi)$, independently of the base point $a$.

Proof. First, $c_{a}^{1}$ is a cocycle:

$$
d c_{a}^{1}(\beta, \gamma)=J_{\beta a}^{a}\left(\beta \cdot J_{\gamma a}^{a}\right)\left(J_{\beta \gamma a}^{a}\right)^{-1}=J_{\beta a}^{a} J_{\beta \gamma a}^{\beta a} J_{a}^{\beta \gamma a}=1 .
$$

Second, $c_{a}^{1}$ and $c_{b}^{1}$ are homologous:

$$
c_{a}^{1}(\gamma)=J_{\gamma a}^{a}=J_{b}^{a} J_{\gamma b}^{b} J_{\gamma a}^{\gamma b}=J_{b}^{a} c_{b}^{1}(\gamma)\left(\gamma \cdot J_{b}^{a}\right)^{-1} .
$$

Definition 2.9. A noncommutative modular symbol is a nonabelian cohomology class in $H^{1}(\Gamma, \Pi)$, with representative

$$
c_{a}^{1}(\gamma)=J_{\gamma a}^{a},
$$

\section{Iterated integrals on membranes}

Iterated integrals on membranes are a higher-dimensional analogue of iterated path integrals. This technical tool was used in [Horozov 2014b] for constructing multiple Dedekind zeta values and in [Horozov 2014c] for proving new and classical reciprocity laws on algebraic surfaces. It appeared first in the preprint [Horozov 2006] for the purpose of noncommutative Hilbert modular symbols.

3A. Definition and properties. Let $\mathbb{W}^{1}$ be the upper half-plane. Let $\mathbb{H}^{2}$ be a product of two upper half-planes. We are interested in the action of $\mathrm{GL}_{2}(K)$, where $K$ is a real quadratic field. This group acts on $\mathbb{H}^{2}$ by linear fractional transformations. It is convenient to introduce cusp points $\mathbb{P}^{1}(K)$ as boundary points of $\mathbb{W}^{2}$. 
Let $\omega_{1}, \ldots, \omega_{m}$ be holomorphic 2-forms on $\mathbb{M}^{2}$ which are continuous at the cusps $\mathbb{P}^{1}(K)$. Let

$$
g:[0,1]^{2} \rightarrow \mathbb{H}^{2} \cup \mathbb{P}^{1}(K)
$$

be a continuous map which is smooth almost everywhere. Denote by $F^{1}$ and $F^{2}$ the following coordinatewise foliations: for any $a \in[0,1]$, define the leaves

$$
F_{a}^{1}=\left\{\left(t_{1}, t_{2}\right) \in[0,1]^{2} \mid t_{1}=a\right\} \quad \text { and } \quad F_{a}^{2}=\left\{\left(t_{1}, t_{2}\right) \in[0,1]^{2} \mid t_{2}=a\right\} .
$$

Definition 3.1. We call the above map $g:[0,1]^{2} \rightarrow \mathbb{M}^{2} \cup \mathbb{P}^{1}(K)$ a membrane on $\mathbb{Q}^{2}$ if it is a continuous and piecewise differentiable map such that $g\left(F_{a}^{1}\right)$ and $g\left(F_{a}^{2}\right)$ belong to a finite union of holomorphic curves in $\mathbb{Q}^{2} \cup \mathbb{P}^{1}(K)$ for all constants $a$.

Similarly, we define a membrane of a Hilbert modular variety. Let $\omega_{1}, \ldots, \omega_{m}$ be holomorphic 2-forms on $Y_{\Gamma}=\mathbb{U}^{2} / \Gamma$ which are continuous at the cusps $\mathbb{P}^{1}(K) / \Gamma$. Let

$$
g:[0,1]^{2} \rightarrow X_{\Gamma}
$$

be a continuous map which is smooth almost everywhere, where $X_{\Gamma}=\mathbb{M}^{2} \cup \mathbb{P}^{1}(K)$. Let $f_{i}: X_{\Gamma} \rightarrow \mathbb{P}^{1}(\mathbb{C})$ for $i=1,2$ be two algebraically independent rational functions on the Hilbert modular surface $X_{\Gamma}$. Denote by $F^{1}$ and $F^{2}$ the following coordinatewise foliations: for any $a \in[0,1]$, define the leaves

$$
F_{a}^{1}=\left\{\left(t_{1}, t_{2}\right) \in[0,1]^{2} \mid t_{1}=a\right\} \quad \text { and } \quad F_{a}^{2}=\left\{\left(t_{1}, t_{2}\right) \in[0,1]^{2} \mid t_{2}=a\right\} .
$$

Let also

$$
P_{x}^{1}=\left\{P \in X_{\Gamma} \mid f_{1}(P)=x\right\} \quad \text { and } \quad P_{x}^{2}=\left\{P \in X_{\Gamma} \mid f_{2}(P)=x\right\} .
$$

Definition 3.2. We call the above map $g:[0,1]^{2} \rightarrow X_{\Gamma}$ a membrane on $X_{\Gamma}$ if it is a continuous and piecewise differentiable map such that for each $a$ there are $x_{1}$ and $x_{2}$ such that $g\left(F_{a}^{1}\right) \subset P_{x_{1}}^{1}$ and $g\left(F_{a}^{2}\right) \subset P_{x_{2}}^{2}$.

We define three types of iterated integrals over membranes - type $\boldsymbol{a}$, type $\boldsymbol{b}$ and type $\boldsymbol{c}$. Type $\boldsymbol{a}$ consists of linear iterations, while type $\boldsymbol{b}$ is more general and involves permutations. Type $\boldsymbol{a}$ is less general, but more intuitive. The advantage of type $\boldsymbol{b}$ is that it satisfies integral shuffle relation (Theorem 3.21). In other words a product of two integrals of type $\boldsymbol{b}$ can be expresses as a finite sum of iterated integrals over membranes of type $\boldsymbol{b}$. However, one might not be able to express a product of two integrals of type $\boldsymbol{a}$ as a sum of finitely many integrals of type $\boldsymbol{a}$. Both type $\boldsymbol{a}$ and type $\boldsymbol{b}$ are defined on a product of two upper half-planes. Type $\boldsymbol{c}$ 
is defined on a Hilbert modular surface; that is, on a quotient of a product of upper half-planes by an arithmetic group which is commensurable to $\mathrm{SL}_{2}\left(\mathbb{O}_{K}\right)$. Type $c$ also satisfies a shuffle product, that is, a product of two integrals of this type can be expresses a finite sum of such integrals.

Definition 3.3 (type $\boldsymbol{a}$, ordered iteration over membranes). Let

$$
g:[0,1]^{2} \rightarrow \mathbb{M}^{2} \cup \mathbb{P}^{1}(K)
$$

be a membrane on $\mathbb{H}^{2} \cup \mathbb{P}^{1}(K)$. Then define

$$
\int_{g} \omega_{1} \cdots \omega_{m}=\int_{D} \bigwedge_{j=1}^{m} g^{*} \omega_{i}\left(t_{1, j}, t_{2, j}\right)
$$

where

$D=\left\{\left(t_{1,1}, \ldots, t_{2, m}\right) \in[0,1]^{2 m} \mid 0 \leq t_{1,1} \leq \cdots \leq t_{1, m} \leq 1,0 \leq t_{2,1} \leq \cdots \leq t_{2, m} \leq 1\right\}$.

Definition 3.4 (type $\boldsymbol{b}$, two permutations). Let

$$
g:[0,1]^{2} \rightarrow \mathbb{M}^{2} \cup \mathbb{P}^{1}(K)
$$

be a membrane on $\mathbb{M}^{2} \cup \mathbb{P}^{1}(K)$, and let $\rho_{1}, \rho_{2}$ be two permutations of the set $\{1,2, \ldots, m\}$. Then define

$$
\int_{g}^{\rho_{1}, \rho_{2}} \omega_{1} \cdots \omega_{m}=\int_{D} \bigwedge_{j=1}^{m} g^{*} \omega_{j}\left(t_{1, \rho_{1}(j)}, t_{2, \rho_{2}(j)}\right),
$$

where

$D=\left\{\left(t_{1,1}, \ldots, t_{2, m}\right) \in[0,1]^{2 m} \mid \leq t_{1,1} \leq \cdots \leq t_{1, m} \leq 1,0 \leq t_{2,1} \leq \cdots \leq t_{2, m} \leq 1\right\}$.

Definition 3.5 (type $c$, two permutations). Let

$$
g:[0,1]^{2} \rightarrow X_{\Gamma}
$$

be a membrane on the Hilbert modular surface $X_{\Gamma}=\left(\mathbb{M}^{2} \cup \mathbb{P}^{1}(K)\right) / \Gamma$, and let $\rho_{1}, \rho_{2}$ be two permutations of the set $\{1,2, \ldots, m\}$. Then define

$$
\int_{g}^{\rho_{1}, \rho_{2}} \omega_{1} \cdots \omega_{m}=\int_{D} \bigwedge_{j=1}^{m} g^{*} \omega_{j}\left(t_{1, \rho_{1}(j)}, t_{2, \rho_{2}(j)}\right)
$$

where

$D=\left\{\left(t_{1,1}, \ldots, t_{2, m}\right) \in[0,1]^{2 m} \mid 0 \leq t_{1,1} \leq \cdots \leq t_{1, m} \leq 1,0 \leq t_{2,1} \leq \cdots \leq t_{2, m} \leq 1\right\}$. 
Examples (iterated integrals of type b). Let $\alpha_{i}\left(t_{1}, t_{2}\right)=g^{*} \omega_{i}\left(t_{1}, t_{2}\right)$. Denote by (1) the trivial permutation and by (1 2 ) the permutation exchanging 1 and 2 .

(1) The four diagrams
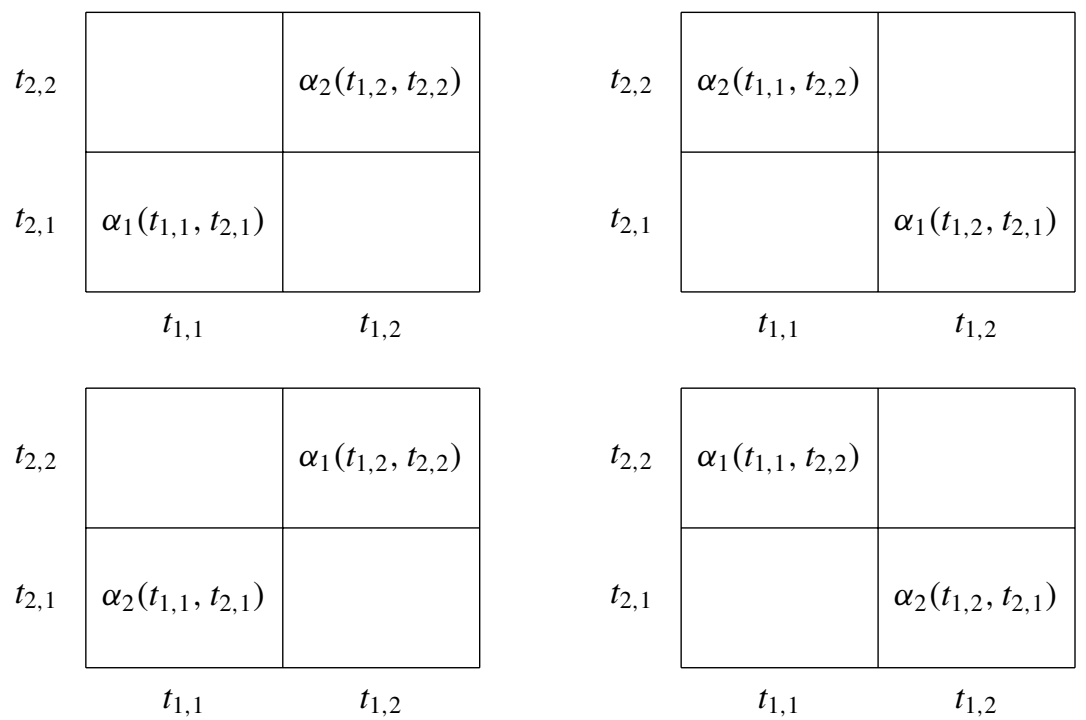

correspond, respectively, to the integrals

$$
\begin{array}{lll}
\int_{g}^{(2),(1)} \omega_{1} \cdot \omega_{2}, & & \int_{g}^{(12),(1)} \omega_{1} \cdot \omega_{2}, \\
\int_{g}^{(12),(12)} \omega_{1} \cdot \omega_{2}, & \int_{g}^{(1),(12)} \omega_{1} \cdot \omega_{2} .
\end{array}
$$

(2) The diagram

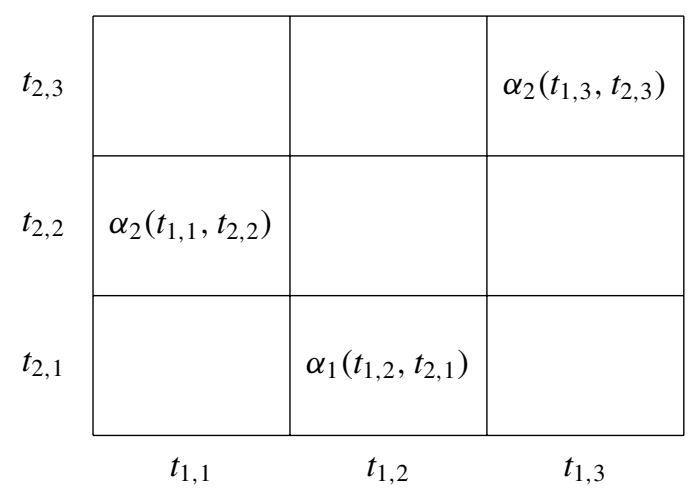

corresponds to the integral

$$
\int_{g}^{(12),(1)} \omega_{1} \cdot \omega_{2} \cdot \omega_{3} .
$$


Remark 3.6. Let us give more intuition for Definition 3.4. Each of the differential forms $g^{*} \omega_{1}, \ldots, g^{*} \omega_{m}$ has two arguments. Consider the set of first arguments for each of the differential forms $g^{*} \omega_{1}, \ldots, g^{*} \omega_{m}$. They are ordered as

$$
0<t_{1,1}<t_{1,2}<\cdots<t_{1, m}<1
$$

(they are the coordinates of the domain $D$ ). Since $g^{*} \omega_{j}$ depends on $t_{1, \rho_{1}(j)}$, we have that $t_{1, k}$ is an argument of $g^{*} \omega_{\rho_{1}^{-1}(k)}$, where $k=\rho_{1}(j)$. Then we can order the differential forms $g^{*} \omega_{1}, \ldots, g^{*} \omega_{m}$ according to the order of their first arguments given by the inequalities (3), which is

$$
g^{*} \omega_{\rho_{1}^{-1}(1)}, g^{*} \omega_{\rho_{1}^{-1}(2)}, \ldots, g^{*} \omega_{\rho_{1}^{-1}(m)} .
$$

Similarly, we can order the differential forms $g^{*} \omega_{1}, \ldots, g^{*} \omega_{m}$ with respect to the order of their second arguments:

$$
g^{*} \omega_{\rho_{2}^{-1}(1)}, g^{*} \omega_{\rho_{2}^{-1}(2)}, \ldots, g^{*} \omega_{\rho_{2}^{-1}(m)} .
$$

We call the first ordering horizontal and the second ordering vertical.

Now we are going to examine the homotopy of a domain of integration and what that reflects about the integral. Let $g_{s}:[0,1]^{2} \rightarrow \mathbb{M}^{2} \cup \mathbb{P}^{1}(K)$ be a family of membranes such that $g_{s}(0,0)=\infty$ and $g_{s}(1,1)=0$. Assume that the parameter $s$ is in the interval $[0,1]$.

Let $h\left(s, t_{1}, t_{2}\right)=g_{s}\left(t_{1}, t_{2}\right)$ be a homotopy between $g_{0}$ and $g_{1}$. Let

$$
G_{s}:[0,1]^{2 m} \rightarrow\left(\mathbb{M}^{2} \cup \mathbb{P}^{1}(K)\right)^{m}
$$

be the map

$G_{s}\left(t_{1,1}, \ldots, t_{2, m}\right)=\left(g_{s}\left(t_{1, \sigma_{1}(1)}, t_{2, \sigma_{2}(1)}\right), g_{s}\left(t_{1, \sigma_{1}(2)}, t_{2, \sigma_{2}(2)}\right), \ldots, g_{s}\left(t_{1, \sigma_{1}(m)}, t_{2, \sigma(m)}\right)\right)$.

Let $H$ be the induced homotopy between $G_{0}$ and $G_{1}$, defined by

$$
H\left(s, t_{1,1}, \ldots, t_{2, m}\right)=G_{s}\left(t_{1,1}, \ldots, t_{2, m}\right) .
$$

We define diagonals in the domain $D \subset(0,1)^{2 m}$, where

$$
\begin{aligned}
D=\left\{\left(t_{1,1}, t_{2,1}, \ldots, t_{1, m}, t_{2, m}\right) \in(0,1)^{2 m} \mid 0\right. & \leq t_{1,1} \leq t_{1,2} \leq \cdots \leq t_{1, m} \leq 1 \\
& \text { and } \left.0 \leq t_{2,1} \leq t_{2,2} \leq \cdots \leq t_{2, m} \leq 1\right\} .
\end{aligned}
$$

We define $D_{1, k}$ for $k=0, \ldots, m$ by $D_{1,0}=\left.D\right|_{t_{1,1}=0}, D_{1, k}=\left.D\right|_{t_{1, k}=t_{1, k+1}}$ for $k=$ $1, \ldots, m-1$ and $D_{1, m}=\left.D\right|_{t_{1, m}=1}$. Similarly, we define $D_{2, k}$ for $k=0, \ldots, m$ by $D_{2,0}=\left.D\right|_{t_{2,1}=0}, D_{2, k}=\left.D\right|_{t_{2, k}=t_{2, k+1}}$ for $k=1, \ldots, m-1$ and $D_{2, m}=\left.D\right|_{t_{2, m}=1}$. 
For iterated integrals of types $\boldsymbol{a}$ and $\boldsymbol{b}$, we define diagonals in $V=\left(\mathbb{R}^{2} \cup \mathbb{P}^{1}(K)\right)^{m}$. We denote a generic coordinate of $V=\left(\mathbb{R}^{2} \cup \mathbb{P}^{1}(K)\right)^{m}$ by $\left(z_{1,1}, z_{2,1}, \ldots, z_{1, m}, z_{2, m}\right)$ For $k=1, \ldots, m-1$, let $V_{1, k}=\left.V\right|_{z_{1, k}=z_{1, k+1}}$. Let also $V_{1,0}=\left.V\right|_{z_{1,1}=0}$ and $V_{1, m}=$ $\left.V\right|_{z_{1, m}=1}$. Similarly, for $k=1, \ldots, m-1$, let $V_{2, k}=\left.V\right|_{z_{2, k}=z_{2, k+1}}$. Let also $V_{2,0}=$ $\left.V\right|_{z_{2,1}=0}$ and $V_{2, m}=\left.V\right|_{z_{2, m}=1}$.

For iterated integrals of type $\boldsymbol{c}$, we define "diagonals" as fibers product of schemes corresponding to certain varieties (for fiber products of schemes one may look at the book [Hartshorne 1977]). Occasionally, it will be more natural to realize multiple fiber products as finite limits in the category of schemes of finite type over $\mathbb{C}$. Let $X_{i, j}=X_{\Gamma}$ for $i, j=1, \ldots, n$. Let $V$ be the universal scheme (finite limit) that maps to $X_{i j}$ for each $i$ and $j$ as a part of a commutative diagram. The commutative diagram is defined as follows: $X_{i, j}$ and $X_{i+1, j}$ both map to $\mathbb{P}^{1}(\mathbb{C})$ via the morphism $f_{1}$ for $1 \leq i \leq n-1$ and all $j$, and $X_{i, j}$ and $X_{i, j+1}$ both map to $\mathbb{P}^{1}(\mathbb{C})$ via the morphism $f_{2}$ for $1 \leq j \leq n-1$ and all $i$. Define the following:

- Let $V_{1,0}$ be the subscheme of $V$ defined by putting $\mathbb{P}^{1}(\mathbb{C})$ in the place of $X_{1, j}$, so that $f_{1}: X_{1, j} \rightarrow \mathbb{P}^{1}(\mathbb{C})$ is replaced by the identity map and the corresponding $f_{2}: X_{1, j} \rightarrow \mathbb{P}^{1}(\mathbb{C})$ is deleted.

- Let $V_{2,0}$ be the subscheme of $V$ defined by putting $\mathbb{P}^{1}(\mathbb{C})$ in the place of $X_{i, 1}$, so that $f_{2}: X_{1, j} \rightarrow \mathbb{P}^{1}(\mathbb{C})$ is replaced by the identity map and the corresponding $f_{1}: X_{1, j} \rightarrow \mathbb{P}^{1}(\mathbb{C})$ is deleted.

- Let $V_{1, n}$ be the subscheme of $V$ defined by putting $\mathbb{P}^{1}(\mathbb{C})$ in the place of $X_{n, j}$, so that $f_{1}: X_{n, j} \rightarrow \mathbb{P}^{1}(\mathbb{C})$ is replaced by the identity map and the corresponding $f_{2}: X_{n, j} \rightarrow \mathbb{P}^{1}(\mathbb{C})$ is deleted.

- Let $V_{2, n}$ be the subscheme of $V$ defined by putting $\mathbb{P}^{1}(\mathbb{C})$ in the place of $X_{i, n}$, so that $f_{2}: X_{n, j} \rightarrow \mathbb{P}^{1}(\mathbb{C})$ is replaced by the identity map and the corresponding $f_{1}: X_{n, j} \rightarrow \mathbb{P}^{1}(\mathbb{C})$ is deleted.

- Additionally, let $V_{1, i}$ be the subscheme of $V$ obtained by replacing each factor $X_{i, j} \times_{\mathbb{P}^{1}(\mathbb{C})} X_{i+1, j}$ by the corresponding diagonal for fixed $i$ and for all $j$.

- Finally, let $V_{2, j}$ be the subscheme of $V$ obtained by replacing each factor $X_{i, j} \times_{\mathbb{P}^{1}(\mathbb{C})} X_{i, j+1}$ by the corresponding diagonal for fixed $j$ and all $i$.

Theorem 3.7 (homotopy invariance theorem I). The iterated integrals on membranes from Definition 3.4 (of type $\boldsymbol{b}$ ) are homotopy-invariant with respect to homotopies that preserve the boundary of the membrane.

Proof. Let

$$
\Omega=\bigwedge_{j=1}^{m} \omega_{j}\left(z_{1, \sigma_{1}(j)}, z_{2, \sigma_{2}(j)}\right) .
$$


Note that $\Omega$ is a closed form, since $\omega_{i}$ is a form of top dimension. By Stokes' theorem, we have

$$
\begin{aligned}
0= & \int_{s=0}^{s=1} \int_{D} H^{*} d \Omega \\
= & \int_{D} G_{1}^{*} \Omega-\int_{D} G_{0}^{*} \Omega \\
& \pm \int_{s=0}^{s=1} \sum_{k=1}^{m-1}\left(\int_{D_{1, k}} \pm \int_{D_{2, k}}\right) H^{*} \Omega \\
& \pm \int_{s=0}^{s=1}\left(\int_{D_{1,0}} \pm \int_{D_{2,0}}\right) H^{*} \Omega \\
& \pm \int_{s=0}^{s=1}\left(\int_{D_{1, m}} \pm \int_{D_{2, m}}\right) H^{*} \Omega .
\end{aligned}
$$

We want to show that the difference in the terms in (5) is zero. It is enough to show that each of the terms (6), (7) and (8) are zero. If $z_{1, k}=z_{1, k+1}$ for types $\boldsymbol{a}$ and $\boldsymbol{b}$ (or on $V_{1, k}$ for type $\boldsymbol{c}$ ), then the wedge of the corresponding differential forms will vanish. Thus the terms in (6) are zero. If $z_{1}=0$ then $d t_{1}=0$, defined via the pullback $H^{*}$. Then the terms (7) are equal to zero. Similarly, we obtain that the last integral (8) vanishes.

Let $A$ be a 2-dimensional manifold with corners in $[0,1]^{2}$. We recall the domain of integration

$$
D=\left\{\left(t_{1,1}, \ldots, t_{2, m}\right) \in[0,1]^{2 m} \mid 0 \leq t_{1,1} \leq \cdots \leq t_{1, m} \leq 1,0 \leq t_{2,1} \leq \cdots \leq t_{2, m} \leq 1\right\} .
$$

Let us define

$$
A^{D}=\left\{\left(t_{1,1}, \ldots, t_{2, m}\right) \in D \mid\left(t_{1, i}, t_{2, j}\right) \in A \text { for } i, j=1, \ldots, m\right\}
$$

Let $\rho_{1}$ and $\rho_{2}$ be two permutations of $m$ elements. We define a function on $A^{D}$ $G\left(t_{1,1}, \ldots, t_{2, m}\right)=\left(g\left(t_{1, \rho_{1}(1)}, t_{2, \rho_{2}(1)}\right), g\left(t_{1, \rho_{1}(2)}, t_{2, \rho_{2}(2)}\right), \ldots, g\left(t_{1, \rho_{1}(m)}, t_{2, \rho(m)}\right)\right)$. Recall that

$$
\Omega=\bigwedge_{j=1}^{m} \omega_{j}\left(z_{1, \rho_{1}(j)}, z_{2, \rho_{2}(j)}\right) .
$$

Definition 3.8. With the above notation, we define an iterated integral over a membrane of type $\boldsymbol{b}$ restricted to a domain $U=g(A)$ by

$$
b \int_{g, U}^{\rho_{1}, \rho_{2}} \omega_{1} \cdots \omega_{m}=\int_{A^{D}} G^{*} \Omega .
$$

Now we are going to define iterated integrals of type $\boldsymbol{c}$ : 
Definition 3.9. Let $\Omega_{0}=\bigwedge_{i, j=1}^{m} \Omega_{i, j}$, where $\Omega_{i, j}=\omega_{i} \delta_{i, j}$ on $X_{i, i} \equiv X_{\Gamma}$ and where $\Omega_{i, j}=1$ for $i \neq j$. Let in $X \rightarrow \prod_{i, j=1}^{n} X_{i, j}$ be the inclusion of the finite limit into the product of the schemes $X_{i, j}$. Let $\Omega=$ in* $^{*} \Omega_{0}$.

With this definition of $\Omega$, we define iterated integrals of type $c$ restricted to a domain $U=g(A)$ by

$$
c \int_{g, U}^{\rho_{1}, \rho_{2}} \omega_{1} \cdots \omega_{m}=\int_{A^{D}} G^{*} \Omega .
$$

Let $A_{1}$ and $A_{2}$ be two manifolds with corners which are subsets of $[0,1]^{2}$, with a common component of the boundary. Let $A=A_{1} \cup A_{2}$. Let $s$ be a map of sets with values 1 or 2 :

$$
s:\{1, \ldots, m\} \rightarrow\{1,2\} .
$$

We define a certain subset $A_{s}^{D}$ of $A^{D}$ as follows: consider the image of the map $G$. It has $m$ coordinates. The first coordinate, $g\left(t_{1, \rho_{1}(1)}, t_{2, \rho_{2}(1)}\right)$, will be restricted to the set $A_{s(1)}$. The second coordinate, $g\left(t_{1, \rho_{1}(2)}, t_{2, \rho_{2}(2)}\right)$, will be restricted to $A_{s(2)}$, and so on, and the last $m$ coordinate $g\left(t_{1, \rho_{1}(m)}, t_{2, \rho_{2}(m)}\right)$ will be restricted to $A_{s(m)}$. Formally, this can be written as

$$
A_{s}^{D}=\left\{\left(t_{1,1}, \ldots, t_{2, m}\right) \in A^{D} \mid\left(t_{1, \rho_{1}(i)}, t_{2, \rho_{2}(i)}\right) \in A_{s(i)} \text { for } i=1, \ldots, m\right\} .
$$

Note that the image of the map $s$ is 1 or 2 .

Definition 3.10. With the above notation, we define an iterated integral of type $\boldsymbol{b}$ or $\boldsymbol{c}$ over two domains $U_{1}$ and $U_{2}$, where $U_{i}=g\left(A_{i}\right)$ and $U=U_{1} \cup U_{2}$, by

$$
\int_{g, U, s}^{\rho_{1} \rho_{2}} \omega_{1} \cdots \omega_{m}=\int_{A_{s}^{D}} G^{*} \Omega
$$

For type $\boldsymbol{b}$ we have that $U$ is in $\mathbb{H}^{2} \cup \mathbb{P}^{1}(K)$ and for type $\boldsymbol{c}$ we have that $U$ is in $X_{\Gamma}=\left(\mathbb{H}^{2} \cup \mathbb{P}^{1}(K)\right) / \Gamma$.

Again we examine the homotopy of iterated integrals on membranes. Now we restrict the domain of integration to a manifold with corners $A$ that is a subset of $[0,1]^{2}$. Assume that for the boundary of a domain $A$, denoted by $\partial A$, we have that $g(\partial A)$ belongs to a finite union of complex analytic curves in $\mathbb{H}^{2}$ for type $\boldsymbol{b}$ and in $X_{\Gamma}$ for type $\boldsymbol{c}$. We call the minimal union of complex analytic (holomorphic) curves such that $g(\partial A)$ belongs to a finite union of complex analytic curves in $\mathbb{Q}^{2}$ for type $\boldsymbol{b}$ and in $X_{\Gamma}$ for type $\boldsymbol{c}$ the complex boundary of $g(\partial A)$.

Theorem 3.11 (homotopy invariance theorem II). Iterated integrals over membranes are homotopy invariant with respect to homotopies that change the boundary $\partial U$ of the domain of integration $U$ so that the boundary varies on a finite union of complex analytic curves. 
Proof. Assume that $g_{0}(\partial A)$ and $g_{1}(\partial A)$ have the same complex boundary. Let $h$ be a homotopy between $g_{0}$ and $g_{1}$, such that for each value of $s$ we have that $h(s, \partial A)$ has the same complex boundary as $h(0, \partial A)=g_{0}(\partial A)$. Let $A \subset B$ be a strict inclusion of disks. Identify $B-A^{\circ}$ with $A \times[0,1]$. Let $i: B-A^{\circ} \rightarrow[0,1] \times \partial A$. Here $A^{\circ}$ is the interior of $A$ and $\partial A$ is the boundary of $A$. Let $\tilde{g}_{0}$ be a map from $B$ to $\mathbb{U}^{2}$ such that $\tilde{g}_{0}(a)=g_{0}(a)$ for $a \in A$ and $\tilde{g}_{0}(b) \in h(i(b))$. Since the restriction of the pullback $\left.\left(\tilde{g}_{0}^{*} \omega_{i}\right)\right|_{B-A}=0$ is mapped to a finite union of complex curves, it vanishes. Therefore

$$
\int_{A} g_{0}^{*} \Omega=\int_{B} \tilde{g}_{0}^{*} \Omega
$$

Let $\tilde{g}_{1}$ be a membrane from $B$ defined by $\tilde{g}_{1}(a)=g_{1}(a)$ for $a \in A$ and $\tilde{g}_{1}(b)=\tilde{g}_{1}(a)$ for $i(b)=(s, a)$. (Note that $i(b) \in[0,1] \times \partial A$.) Again,

$$
\int_{A} g_{1}^{*} \Omega=\int_{B} \tilde{g}_{1}^{*} \Omega
$$

However, the boundary of $B$ is mapped to the same set (pointwise) by both $\tilde{g}_{0}$ and $\tilde{g}_{1}$. Moreover, the homotopy between $g_{0}$ and $g_{1}$ extends to a homotopy between $\tilde{g}_{0}$ and $\tilde{g}_{1}$ that respects the inclusion into the complex boundary. Thus by Theorem 3.7, we have that

$$
\int_{B} \tilde{g}_{0}^{*} \Omega=\int_{B} \tilde{g}_{1}^{*} \Omega
$$

Using (10) and (11), we complete the proof of this theorem.

3B. Generating series. We are going to define two types of generating series type $\boldsymbol{a}$ and type $\boldsymbol{b}$, corresponding to the iterated integrals on membranes of type $\boldsymbol{a}$ and type $\boldsymbol{b}$.

Definition 3.12 (type $\boldsymbol{a}$ ). Let $A$ be a domain in $\mathbb{R}^{2}$. Let $g$ be a membrane. Let $U=g(A) \subset \mathbb{M}^{2}$. And let $\omega_{1}, \ldots, \omega_{m}$ be holomorphic 2-forms on $\mathbb{\boxplus}^{2}$. We define a generating series of type $\boldsymbol{a}$ by

$$
J^{a}(U)=1+\sum_{k=1}^{\infty} \sum_{c:\{1, \ldots, k\} \rightarrow\{1, \ldots, m\}} X_{c(1)} \otimes \cdots \otimes X_{c(k)} \int_{g, U} \omega_{c(1)} \cdots \omega_{c(k)},
$$

where $c:\{1, \ldots, k\} \rightarrow\{1, \ldots, m\}$ is a map of sets.

Consider a map of sets $c:\{1, \ldots, k\} \rightarrow\{1, \ldots, m\}$ and two permutations $\rho_{1}, \rho_{2}$ of $\{1,2, \ldots, k\}$. We call two triples $\left(c^{\prime}, \rho_{1}^{\prime}, \rho_{2}^{\prime}\right)$ and $\left(c^{\prime \prime}, \rho_{1}^{\prime \prime}, \rho_{2}^{\prime \prime}\right)$ equivalent if they are in the same orbit of the permutation group $S_{k}$. That is, $\left(c^{\prime \prime}, \rho_{1}^{\prime \prime}, \rho_{2}^{\prime \prime}\right) \sim\left(c^{\prime}, \rho_{1}^{\prime}, \rho_{2}^{\prime}\right)$ if for some $\tau \in S_{k}$ we have $c^{\prime \prime}=c^{\prime} \tau^{-1}, \rho_{1}^{\prime \prime}=\rho_{1}^{\prime} \tau^{-1}$ and $\rho_{2}^{\prime \prime}=\rho_{2}^{\prime} \tau^{-1}$. Then for the equivalence class of a triple $\left(c, \rho_{1}, \rho_{2}\right)$, we can associate a unique pair $\left(c \circ \rho_{1}, c \circ \rho_{2}\right)$ (which are precisely the indices of the $X$ - and $Y$-variables in (12) 
and (13), respectively.) The reason for using such an equivalence is that the integral in (13) is invariant by the above action of $\tau \in S_{k}$ on the triple $\left(c, \rho_{1}, \rho_{2}\right)$.

Definition 3.13 (ring $R$, values of the generating series). The values of the generation series of iterated integrals on membranes will be in a ring $R$, which we define as follows. Let

$$
R_{0}=\mathbb{C}\left\langle\left\langle X_{1}, Y_{1}, \ldots, X_{m}, Y_{m}\right\rangle\right\rangle / I
$$

be the quotient of the ring of formal power series modulo the two-sided ideal $I$ generated by $X_{i} Y_{j}-Y_{j} X_{i}$ for $i, j=1, \ldots, m$. Let $R \subset R_{0}$ be the subring of formal power series whose monomials have the following property: in every monomial of $R, X_{i}$ occurs as many times as $Y_{i}$.

Definition 3.14 (type b). We define the generating series of type $\boldsymbol{b}$ on $U$ by

$$
\begin{aligned}
J^{b}(U)=1+\sum_{k=1}^{\infty} \sum_{\left(c, \rho_{1}, \rho_{2}\right) / \sim} & X_{c\left(\rho_{1}^{-1}(1)\right)} \otimes \cdots \otimes X_{c\left(\rho_{1}^{-1}(k)\right)} \\
& \otimes Y_{c\left(\rho_{2}^{-1}(1)\right)} \otimes \cdots \otimes Y_{c\left(\rho_{2}^{-1}(k)\right)} \int_{g, U}^{\rho_{1}, \rho_{2}} \omega_{c(1)} \cdots \omega_{c(k)},
\end{aligned}
$$

where the second summation is over all maps of sets $c:\{1, \ldots, k\} \rightarrow\{1, \ldots, m\}$ and all permutations $\rho_{1}, \rho_{2}$ of $k$ elements, up to the above equivalence.

Let $Y_{\Gamma}$ be a Hilbert modular surface. Let $\alpha$ and $\beta$ be two rational functions on $Y_{\Gamma}$. We denote by $D$ the union of the divisors $(\alpha)_{\infty}$ and $(\beta)_{\infty}$ at infinity. Let $F: Y_{\Gamma}-D \rightarrow \mathbb{C}^{2}$ be defined as $F(y)=(\alpha(y), \beta(y))$. Let $g:(0,1)^{2} \rightarrow Y_{\Gamma}-D$ be a membrane, so that the composition $F \circ g$ respects the coordinatewise foliations. Consider the differential forms $\omega_{i}$ from the definition of type $\boldsymbol{b}$. They are invariant under the action of the arithmetic group $\Gamma$. Thus, we can treat them as differential forms on the Hilbert modular variety $Y_{\Gamma}$.

Definition 3.15 (type c). With the new definition of a membrane $g$ and a domain $U \subset Y_{\Gamma}$, we define the generating series of type $c$ by

$$
\begin{aligned}
J^{c}(U)=1+\sum_{k=1}^{\infty} \sum_{\left(c, \rho_{1}, \rho_{2}\right) / \sim} X_{c\left(\rho_{1}^{-1}(1)\right)} \otimes \cdots \otimes X_{c\left(\rho_{1}^{-1}(k)\right)} \\
\otimes Y_{c\left(\rho_{2}^{-1}(1)\right)} \otimes \cdots \otimes Y_{c\left(\rho_{2}^{-1}(k)\right)} \int_{g, U}^{\rho_{1}, \rho_{2}} \omega_{c(1)} \cdots \omega_{c(k)},
\end{aligned}
$$

where the second summation is over all maps of sets $c:\{1, \ldots, k\} \rightarrow\{1, \ldots, m\}$ and all permutations $\rho_{1}, \rho_{2}$ of $k$ elements, up to the above equivalence. 
Definition 3.16 (ring $R^{\prime}$, generating series $J\left(U_{1}, U_{2}\right)$ ). We define a generating series of iterated integrals on two disjoint domain $U_{1}$ and $U_{2}$ (see Definition 3.10). Let $U_{i}=g\left(A_{i}\right)$. Define

$$
\begin{aligned}
& J\left(U_{1}, U_{2}\right){ }_{\infty}^{\infty} \sum_{k=1} \sum_{s:\{1, \ldots, k\} \rightarrow\{1,2\}} \sum_{\left(c, \rho_{1}, \rho_{2}\right) / \sim} X_{c\left(\rho_{1}^{-1}(1)\right), s(1)} \otimes \cdots \otimes X_{c\left(\rho_{1}^{-1}(k)\right), s(k)} \\
& \otimes Y_{c\left(\rho_{2}^{-1}(1)\right), s(1)} \otimes \cdots \otimes Y_{c\left(\rho_{2}^{-1}(k)\right), s(k)} \int_{g, U, s}^{\rho_{1}, \rho_{2}} \omega_{c(1)} \cdots \omega_{c(k)},
\end{aligned}
$$

The generating series takes values in a ring $R^{\prime}$ defined as follows. Let

$$
R_{0}^{\prime}=\mathbb{C}\left\langle\left\langle X_{1,1}, X_{1,2}, Y_{1,1}, Y_{1,2}, \ldots, X_{m, 1}, X_{m, 2}, Y_{m, 1}, Y_{m, 2}\right\rangle\right\rangle / I^{\prime}
$$

be a quotient of the ring of formal power series, where $I^{\prime}$ is the two-sided ideal generated by the Lie commutators of all the $X_{i, j}$ and $Y_{k, l}$. Let $R^{\prime}$ be the subring of $R_{0}^{\prime}$ with the property that in every monomial of $R^{\prime}, X_{i, j}$ occurs as many times as $Y_{i, j}$.

Lemma 3.17. Let $\phi: R^{\prime} \rightarrow R$ be the homomorphism of rings defined by $\phi\left(X_{i, 1}\right)=$ $\phi\left(X_{i, 2}\right)=X_{i}$ and $\phi\left(Y_{i, 1}\right)=\phi\left(Y_{i, 2}\right)=Y_{i}$. If $U=U_{1} \cup U_{2}$ is in $\mathbb{H}^{2} \cup \mathbb{P}^{1}(K)$, then

$$
\phi\left(J\left(U_{1}, U_{2}\right)\right)=J^{b}(U) .
$$

If $U=U_{1} \cup U_{2}$ is in $X_{\Gamma}$, then

$$
\phi\left(J\left(U_{1}, U_{2}\right)\right)=J^{c}(U) .
$$

Proof. After applying the homomorphism $\phi$ the formal variables on the left-hand side become independent of the map $s$. Therefore, we have to examine what happens when we sum over all possible maps $s$. The value $s(i)$ is 1 or 2 . This has the following significance: if $s(i)=1$, then we restrict the form $g^{*} \omega_{c(i)}$ to $A_{1}$ (instead of to $A$ ). Similarly, if $s(i)=2$, we restrict $g^{*} \omega_{c(i)}$ to $A_{2}$. If we add both choices (restriction to $A_{1}$ and restriction to $A_{2}$ ) then we obtain the restriction of $g^{*} \omega_{c(i)}$ to $A=A_{1} \cup A_{2}$. Thus, we obtain the formula

$$
\sum_{s:\{1, \ldots, k\} \rightarrow\{1,2\}} \int_{g, U, s}^{\rho_{1}, \rho_{2}} \omega_{c(1)} \cdots \omega_{c(k)}=\int_{g, U}^{\rho_{1}, \rho_{2}} \omega_{c(1)} \cdots \omega_{c(k)} .
$$

We do the same for every monomial in $R$. That proves the above lemma for the generating series.

3C. Shuffle product of generating series. The regions of integration that we are mostly interested in will be ideal diangles, that is, 2-cells whose boundaries have two vertices and two edges, and ideal triangles. All other regions that we will deal with are going to be a finite union of ideal diangles and ideal triangles. The first 
type of decomposition is based on a union of two diangles with a common vertex. The second type of decomposition will be based on two of the cells (diangles or triangles) with a common edge.

Let $g_{1}$ and $g_{2}$ be two membranes. Let $P=(0,0)$ and $Q=(1,1)$ be the vertices of a diangle $A \subset \mathbb{R}^{2}$ and $Q=(1,1)$ and $R=(2,2)$ be the two points of a diangle $B \subset \mathbb{R}^{2}$. Assume that $A$ lies within the rectangle with vertices $(0,0),(0,1),(1,1),(1,0)$. Similarly, assume that $B$ lies within the rectangle $(1,1),(1,2),(2,2),(2,1)$. Let $U=g(A)$ and $V=g(B)$.

Theorem 3.18. (i) $\int_{g, U \cup V} \omega_{1} \cdots \omega_{m}=\sum_{j=0}^{m} \int_{g, U} \omega_{1} \cdots \omega_{j} \int_{g, V} \omega_{j+1} \cdots \omega_{m}$.

(ii) The generating series of type a from Definition 3.12 satisfies

$$
J^{a}(g ; A \cup B ; \Omega)=J^{a}(g ; A ; \Omega) J^{a}(g ; B ; \Omega) .
$$

The proof of the first statement is essentially the same as the combinatorial proof for composition of paths, when one considers iterated path integrals (see Lemma 2.3). The proof of the second statement combines all compositions into generating series (see Definition 3.12), resembling Manin's approach for the noncommutative modular symbol.

For generating series of type $\boldsymbol{b}$, we have a similar statement:

Definition 3.19. Let $\rho^{\prime}$ and $\rho^{\prime \prime}$ be two permutations of the sets $\{1, \ldots, i\}$ and $\{i+1, \ldots, i+j\}$, respectively. We define the permutation $\rho^{\prime-1} \cup \rho^{\prime \prime-1}$ of $\{1, \ldots, i+j\}$, which acts on $\{1, \ldots, i\}$ as $\rho^{-1}$ and on $\{i+1, \ldots, i+j\}$ as $\rho^{\prime \prime-1}$. We define the set of shuffles of two given permutations, denoted by $\operatorname{sh}\left(\rho^{\prime}, \rho^{\prime \prime}\right)$, as the set of all permutations $\rho$ of the set $\{1,2, \ldots, i+j\}$ such that $\rho^{-1}$ is the composition of a shuffle of sets $\tau \in \operatorname{sh}(i, j)$ (see Definition 2.4) with $\rho^{\prime-1} \cup \rho^{\prime \prime-1}$. That is,

$$
\rho^{-1}=\tau \circ\left(\rho^{\prime-1} \cup \rho^{\prime \prime-1}\right) .
$$

Definition 3.20. We define a shuffle of two monomials

$$
M^{\prime}=X_{c^{\prime}\left(\rho_{1}^{\prime-1}(1)\right)} \otimes \cdots \otimes X_{c^{\prime}\left(\rho_{1}^{\prime-1}(i)\right)} \otimes Y_{c^{\prime}\left(\rho_{2}^{\prime-1}(1)\right)} \otimes \cdots \otimes Y_{c^{\prime}\left(\rho_{2}^{\prime-1}(i)\right)} \int_{g, U^{\prime}}^{\rho_{1}^{\prime}, \rho_{2}^{\prime}} \omega_{c^{\prime}(1)} \cdots \omega_{c^{\prime}(i)}
$$

and

$$
\begin{aligned}
M^{\prime \prime}=X_{c^{\prime \prime}\left(\rho_{1}^{\prime \prime-1}(1)\right)} \otimes \cdots \otimes X_{c^{\prime \prime}\left(\rho_{1}^{\prime \prime-1}(j)\right)} \otimes Y_{c^{\prime \prime}\left(\rho_{2}^{\prime \prime-1}(1)\right)} & \otimes \\
& \cdots \otimes Y_{c^{\prime \prime}\left(\rho_{2}^{\prime \prime-1}(j)\right)} \\
& \times \int_{g, U^{\prime \prime}}^{\rho_{1}^{\prime \prime}, \rho_{2}^{\prime \prime}} \omega_{c^{\prime \prime}(i+1)} \cdots \omega_{c^{\prime \prime}(i+j)},
\end{aligned}
$$

where $\rho_{1}^{\prime}$ and $\rho_{2}^{\prime}$ are permutations of $\{1, \ldots, i\}, c^{\prime}$ is a map of sets $\{1, \ldots, i\} \rightarrow$ $\{1, \ldots, m\}, \rho_{1}^{\prime \prime}$ and $\rho_{2}^{\prime \prime}$ are permutations of $\{i+1, \ldots, i+j\}$, and $c^{\prime \prime}$ is a map of 
sets $\{i+1, \ldots, i+j\} \rightarrow\{1, \ldots, m\}$. By a shuffle product of the monomials $M^{\prime}$ and $M^{\prime \prime}$, we mean the sum

$$
\begin{aligned}
M^{\prime} \times_{\mathrm{Sh}} M^{\prime \prime}= & \sum_{\substack{\rho_{1} \in \operatorname{sh}\left(\rho_{1}^{\prime}, \rho_{1}^{\prime \prime}\right) \\
\rho_{2} \in \operatorname{sh}\left(\rho_{2}^{\prime}, \rho_{2}^{\prime \prime}\right)}} X_{c\left(\rho_{1}^{-1}(1)\right), s(1)} \otimes \cdots \otimes X_{c\left(\rho_{1}^{-1}(i+j)\right), s(i+j)} \\
& \otimes Y_{c\left(\rho_{2}^{-1}(1)\right), s(1)} \otimes \cdots \otimes Y_{c\left(\rho_{2}^{-1}(i+j)\right), s(i+j)} \int_{g, U^{\prime} \cup U^{\prime \prime}, s}^{\rho_{1}, \rho_{2}} \omega_{c(1)} \cdots \omega_{c(i+j)},
\end{aligned}
$$

where $c:\{1, \ldots, i+j\} \rightarrow\{1, \ldots, m\}$ is such that its restriction to the first $i$ elements is $c^{\prime}$ and its restriction to the last $j$ elements is $c^{\prime \prime}$. Here the maps $s$ takes the value 1 on the set $c^{-1}\{1, \ldots, i\}=c^{\prime-1}\{1, \ldots, i\}$ and the value 2 on the set $c^{-1}\{i+1, \ldots, i+j\}=c^{\prime \prime-1}\{i+1, \ldots, i+j\}$.

Theorem 3.21 (shuffle product). For iterated integrals of type $\boldsymbol{b}$ and the corresponding generating series, we have the following shuffle relations:

$$
\int_{g, U}^{\rho_{1}^{\prime}, \rho_{2}^{\prime}} \omega_{1} \cdots \omega_{j} \int_{g, U}^{\rho_{1}^{\prime \prime}, \rho_{2}^{\prime \prime}} \omega_{j+1} \cdots \omega_{m}=\sum_{\substack{\rho_{1} \in \operatorname{sh}\left(\rho_{1}^{\prime}, \rho_{1}^{\prime \prime}\right) \\ \rho_{2} \in \operatorname{sh}\left(\rho_{2}^{\prime}, \rho_{2}^{\prime \prime}\right)}} \int_{g, U}^{\rho_{1}, \rho_{2}} \omega_{1} \cdots \omega_{m} .
$$

(ii)

$$
\int_{g, U^{\prime}}^{\rho_{1}^{\prime}, \rho_{2}^{\prime}} \omega_{1} \cdots \omega_{j} \int_{g, U^{\prime \prime}}^{\rho_{1}^{\prime \prime}, \rho_{2}^{\prime \prime}} \omega_{j+1} \cdots \omega_{m}=\sum_{\substack{\rho_{1} \in \operatorname{sh}\left(\rho_{1}^{\prime}, \rho_{1}^{\prime \prime}\right) \\ \rho_{2} \in \operatorname{sh}\left(\rho_{2}^{\prime}, \rho_{2}^{\prime \prime}\right)}} \int_{g, U, s}^{\rho_{1}, \rho_{2}} \omega_{1} \cdots \omega_{m},
$$

where $s$ is a map from $\{1, \ldots, m\}$ to $\{1,2\}$ such that $\{1, \ldots, j\}$ are mapped to 1 and the remaining elements are mapped to 2.

$$
\begin{aligned}
& \phi\left(J^{b}\left(U^{\prime}\right) \times_{\mathrm{Sh}} J^{b}\left(U^{\prime \prime}\right)\right)=J^{b}\left(U^{\prime} \cup U^{\prime \prime}\right) . \\
& \phi\left(J^{c}\left(U^{\prime}\right) \times_{\mathrm{Sh}} J^{c}\left(U^{\prime \prime}\right)\right)=J^{c}\left(U^{\prime} \cup U^{\prime \prime}\right) .
\end{aligned}
$$

Proof. For part (i), it is useful to consider the two orderings of differential forms, given in Remark 3.6. Note that we need to order the forms both horizontally and vertically in the terminology of that remark. Let us consider first the horizontal order. That is the order with respect to the first variables of the differential forms $g^{*} \omega_{\rho_{1}^{\prime-1}(1)}, \ldots, g^{*} \omega_{\rho_{1}^{\prime-1}(j)}$ and $g^{*} \omega_{\rho_{1}^{\prime \prime-1}(j+1)}, \ldots, g^{*} \omega_{\rho_{1}^{\prime \prime-1}(m)}$, corresponding to the two integrals on the left-hand side of (18). In order to arrange both of the above orderings in one sequence of increasing first arguments, we need to shuffle them (similarly to shuffling a deck of cards). That leads to $\rho_{1} \in \operatorname{sh}\left(\rho_{1}^{\prime}, \rho_{1}^{\prime \prime}\right)$ (see Definition 3.19). We proceed similarly with the second arguments and the permutations $\rho_{2}^{\prime}, \rho_{2}^{\prime \prime}$ and $\rho_{2}$.

For (ii), apply the equality from part (i) with the differential forms $g^{*} \omega_{1}, \ldots, g^{*} \omega_{j}$ multiplied by the function $\mathbf{1}_{A^{\prime}}$, defined by

$$
\mathbf{1}_{A^{\prime}}(x)= \begin{cases}1 & \text { for } x \in A^{\prime}, \\ 0 & \text { for } x \notin A^{\prime},\end{cases}
$$


and the differential forms $g^{*} \omega_{j+1}, \ldots, g^{*} \omega_{m}$ multiplied by $\mathbf{1}_{\mathbf{A}^{\prime \prime}}$.

For part (iii), we are going to establish similar relation among generating series as elements of $R^{\prime}$. Applying the homomorphism $\phi: R^{\prime} \rightarrow R$ from Lemma 3.17, we obtain the desired equality. Every monomial from $J\left(U_{1}\right)$ is of the form

$$
M^{\prime}=X_{c^{\prime}\left(\rho_{1}^{\prime-1}(1)\right)} \otimes \cdots \otimes X_{c^{\prime}\left(\rho_{1}^{\prime-1}(i)\right)} \otimes Y_{c^{\prime}\left(\rho_{2}^{\prime-1}(1)\right)} \otimes \cdots \otimes Y_{c^{\prime}\left(\rho_{2}^{\prime-1}(i)\right)} \int_{g, U^{\prime}}^{\rho_{1}^{\prime}, \rho_{2}^{\prime}} \omega_{c^{\prime}(1)} \cdots \omega_{c^{\prime}(i)}
$$

and similarly every monomial from $J\left(U_{2}\right)$ is of the form

$$
\begin{aligned}
M^{\prime \prime}=X_{c^{\prime \prime}\left(\rho_{1}^{\prime \prime 1}(1)\right)} \otimes \cdots \otimes X_{c^{\prime \prime}\left(\rho_{1}^{\prime \prime 1}(j)\right)} \otimes Y_{c^{\prime \prime}\left(\rho_{2}^{\prime \prime-1}(1)\right)} & \otimes \\
& \cdots \otimes Y_{c^{\prime \prime}\left(\rho_{2}^{\prime \prime-1}(j)\right)} \\
& \times \int_{g, U^{\prime \prime}}^{\rho_{1}^{\prime \prime}, \rho_{2}^{\prime \prime}} \omega_{c^{\prime \prime}(i+1)} \cdots \omega_{c^{\prime \prime}(i+j)},
\end{aligned}
$$

where $\rho_{1}^{\prime}$ and $\rho_{2}^{\prime}$ are permutations of $\{1, \ldots, i\}, c^{\prime}$ is a map of sets $\{1, \ldots, i\} \rightarrow$ $\{1, \ldots, m\}, \rho_{1}^{\prime \prime}$ and $\rho_{2}^{\prime \prime}$ are permutations of $\{i+1, \ldots, i+j\}$, and $c^{\prime \prime}$ is a map of sets $\{i+1, \ldots, i+j\} \rightarrow\{1, \ldots, m\}$. We take the shuffle product of the monomials $M^{\prime}$ and $M^{\prime \prime}$ (see Definition 3.20):

$$
\begin{aligned}
M^{\prime} \times{ }_{\mathrm{Sh}} M^{\prime \prime}= & \sum_{\substack{\rho_{1} \in \operatorname{sh}\left(\rho_{1}^{\prime}, \rho_{1}^{\prime \prime}\right) \\
\rho_{2} \in \operatorname{sh}\left(\rho_{2}^{\prime}, \rho_{2}^{\prime \prime}\right)}} X_{c\left(\rho_{1}^{-1}(1)\right), s(1)} \otimes \cdots \otimes X_{c\left(\rho_{1}^{-1}(i+j)\right), s(i+j)} \\
& \otimes Y_{c\left(\rho_{2}^{-1}(1)\right), s(1)} \otimes \cdots \otimes Y_{c\left(\rho_{2}^{-1}(i+j)\right), s(i+j)} \int_{g, U, s}^{\rho_{1}, \rho_{2}} \omega_{c(1)} \cdots \omega_{c(i+j)},
\end{aligned}
$$

where the map $s$ takes the value 1 on the set $c^{-1}\{1, \ldots, i\}$ and the value 2 on the set $c^{-1}\{i+1, \ldots, i+j\}$. This determines the map $s$ uniquely.

In order to complete the proof, we have to show that every monomial in $J\left(U_{1}, U_{2}\right)$ can be obtained in exactly one way as a result (on the right-hand side) of a shuffle product of a pair of monomials $\left(M_{1}, M_{2}\right)$ from $J\left(U_{1}\right)$ and $J\left(U_{2}\right)$. Every monomial from $J\left(U_{1}, U_{2}\right)$ is characterized by two permutation $\rho_{1}, \rho_{2}$ and two maps of sets $c:\{1, \ldots, k\} \rightarrow\{1, \ldots, m\}$ and $s:\{1, \ldots, k\} \rightarrow\{1,2\}$. Let $i$ be the number of elements in $s^{-1}(1)$ and $j$ the number of elements in $s^{-1}(2)$. Then $i+j=k$. Then $i$ is the number of differential forms among $g^{*} \omega_{c(1)}, \cdots, g^{*} \omega_{c(k)}$ which are restricted to the set $A_{1}$. The remaining $j$ differential forms are restricted to $A_{2}$. Also, every permutation $\rho_{1}$ can be written in an unique way as a composition of a shuffle $\tau_{1} \in \operatorname{sh}(i, j)$ and two disjoint permutations $\rho_{1}^{\prime}$ and $\rho_{1}^{\prime \prime}$ of $i$ and of $j$ elements, respectively (see Definition 3.19). Similarly, $\rho_{2}$ can be written in a unique way as a product of a shuffle $\tau_{2} \in \operatorname{sh}(i, j)$ and two disjoint permutation $\rho_{2}^{\prime}$ and $\rho_{2}^{\prime \prime}$. The map of sets $c_{1}$ is defined as a restriction of the map $c$ to the image of $\rho_{1}^{\prime}$. Similarly, the map $c_{2}$ is defined as a restriction of the map $c$ to the image of $\rho_{1}^{\prime \prime}$. Now we can define the monomials $M^{\prime}$ and $M^{\prime \prime}$ in $J\left(U_{1}\right)$ and $J\left(U_{2}\right)$ based on the triples $\rho_{1}^{\prime}, \rho_{2}^{\prime}, c^{\prime}$ 
and $\rho_{1}^{\prime \prime}, \rho_{2}^{\prime \prime}, c^{\prime \prime}$, respectively. Such monomials are unique. One can show that the shuffle product of $M^{\prime}$ and $M^{\prime \prime}$ contains the monomial in $J\left(U_{1}, U_{2}\right)$ that we started with exactly once. The proof of part (iii) is complete after applying Lemma 3.17.

\section{Hilbert modular symbols}

In this section, we recall the Hilbert modular group and its action on the product of two upper half-planes. Then we define commutative Hilbert modular symbols (Section 4A) and its pairing with the cohomology of the Hilbert modular surface (Section 4B). In Sections 4C and 4D, we define noncommutative Hilbert modular symbols (Definition 4.13) as generating series of iterated integrals over membranes of type $\boldsymbol{b}$. We also examine relations among the noncommutative Hilbert modular symbols (Theorem 4.12), which we interpret as cocycle conditions or as a difference by a coboundary (Conjecture 4.14). In Section 4E, we consider a two-category $C$ with a sheaf $J$ on $C$. Then the noncommutative Hilbert modular symbol is a sheaf on a two-category. This is done in order to give a plausible approach to defining a suitable noncommutative cohomology set. In Section 4F, we make explicit computations and compare them to computations for multiple Dedekind zeta values.

4A. Commutative Hilbert modular symbols. In this subsection, we define commutative Hilbert modular symbols, using geodesics, geodesic triangles and geodesic diangles. Then, we prove certain relations among the commutative Hilbert modular symbols, which are generalized to relations among noncommutative Hilbert modular symbols (Section 4D).

Let $K=\mathbb{Q}(\sqrt{d})$ be a real quadratic extension of $\mathbb{Q}$. Then the ring of integers in $K$ is

$$
\mathcal{O}_{K}= \begin{cases}\mathbb{Z}[(1+\sqrt{d}) / 2] & \text { for } d=1 \bmod 4, \\ \mathbb{Z}[\sqrt{d}] & \text { for } d=2,3 \bmod 4 .\end{cases}
$$

Then $\Gamma=\mathrm{SL}_{2}\left(\mathrm{O}_{K}\right)$ is called a Hilbert modular group. Let $\gamma \in \Gamma$. We recall the action of $\gamma$ on a product of two upper half-planes $\mathbb{U}^{2}$. Let

$$
\gamma=\gamma_{1}=\left(\begin{array}{ll}
a_{1} & b_{1} \\
c_{1} & d_{1}
\end{array}\right)
$$

Let $a_{2}, b_{2}, c_{2}, d_{2}$ be the Galois conjugates of $a_{1}, b_{1}, c_{1}, d_{1}$, respectively. Let us define $\gamma_{2}$ by

$$
\gamma_{2}=\left(\begin{array}{ll}
a_{2} & b_{2} \\
c_{2} & d_{2}
\end{array}\right)
$$

Let $z=\left(z_{1}, z_{2}\right)$ be any point of the product of two upper half-planes $\mathbb{T}^{2}$. 
For an element $\gamma \in \mathrm{GL}_{2}(K)$, we define the following action: If $\operatorname{det} \gamma$ is totally positive, that is $\operatorname{det} \gamma_{1}>0$ and $\operatorname{det} \gamma_{2}>0$, then the action of $\gamma$ on $z=\left(z_{1}, z_{2}\right) \in \mathbb{U}^{2}$ is essentially the same as for $\gamma \in \mathrm{SL}_{2}(K)$, namely,

$$
\gamma z=\left(\gamma_{1} z_{1}, \gamma_{2} z_{2}\right)
$$

where

$$
\gamma_{1} z_{1}=\frac{a_{1} z_{1}+b_{1}}{c_{1} z_{1}+d_{1}} \quad \text { and } \quad \gamma_{2} z_{2}=\frac{a_{2} z_{2}+b_{2}}{c_{2} z_{2}+d_{2}}
$$

are linear fractional transforms. If $\operatorname{det} \gamma$ is totally negative, that is, $\operatorname{det} \gamma_{1}<0$ and $\operatorname{det} \gamma_{2}<0$, then we define

$$
\gamma z=\left(-\frac{a_{1} \bar{z}_{1}+b_{1}}{c_{1} \bar{z}_{1}+d_{1}},-\frac{a_{2} \bar{z}_{2}+b_{2}}{c_{2} \bar{z}_{2}+d_{2}}\right) .
$$

Similarly if one of the embeddings of det $\gamma$ is positive and the other is negative, for example, $\operatorname{det} \gamma_{1}>0$ and $\operatorname{det} \gamma_{2}<0$, e.g., for $\operatorname{det} \gamma=\sqrt{d}$, then

$$
\gamma z=\left(\frac{a_{1} z_{1}+b_{1}}{c_{1} z_{1}+d_{1}},-\frac{a_{2} \bar{z}_{2}+b_{2}}{c_{2} \bar{z}_{2}+d_{2}}\right) \text {. }
$$

We add cusp points $\mathbb{P}^{1}(K)$ to $\mathbb{M}^{2}$. Then the quotient $\mathrm{SL}_{2}\left(\mathbb{O}_{K}\right) \backslash\left(\mathbb{P}^{1}(K) \cup \mathbb{M}^{2}\right)$ is compact.

We are going to carefully examine geodesics joining the cusps 0,1 and $\infty$.

Let $z_{0}, z_{1}, z_{\infty}$ be three distinct cusp points. There is a unique $\gamma \in \mathrm{PGL}_{2}(K)$ that sends $z_{0}, z_{1}$ and $z_{\infty}$ to 0,1 and $\infty$, respectively.

Let

$$
i: \mathbb{H} \rightarrow \mathbb{H}^{2}, \quad i(x)=(x, x)
$$

be the diagonal map and $\Delta$ be its image. Consider the Hirzebruch-Zagier divisor $X=\gamma^{*} \Delta$. It is an analytic curve that passes through the points $z_{0}, z_{1}$ and $z_{\infty}$. Then $X$ is a holomorphic curve in $\mathbb{H}^{2}$ if $\operatorname{det} \gamma$ is totally positive or totally negative. If $\operatorname{det} \gamma$ is not totally positive or totally negative, then $X$ is a holomorphic curve in $\mathbb{M}^{1} \times \mathbb{\mathbb { M }}^{1} \cup \mathbb{P}^{1}(K)$; in other words, it is an antiholomorphic curve in $\mathbb{H}^{2}$, such as $z_{1}=-\bar{z}_{2}$. Let $\Delta_{X}=\gamma^{*} \Delta$ be the pullback of the geodesic triangle $\Delta$ formed by the points $0,1, \infty$ in the analytic curve $X$.

Given four points on the boundary in $\mathbb{H}^{2} \cup \mathbb{P}^{1}(K)$, we are tempted to consider them as vertices of a geodesic tetrahedron in $\mathbb{M}^{2} \cup \mathbb{P}^{1}(K)$, whose faces are triangles of the type $\Delta_{X}$. However, there is one problem that we encounter: Two distinct cusps could be connected by two different geodesics in $\mathbb{T}^{2} \cup \mathbb{P}^{1}(K)$. In particular, two triangles from the faces of the "tetrahedron" might not have a common edge, but only two common vertices. Thus, we are led to consider a thickened tetrahedron with two types of faces on the boundary: the first type is an ideal triangle that we have just defined and the other type is an ideal diangle - a union of geodesics 
connecting two fixed points, which has the homotopy type of a disc with two vertices and two edges. The two edges of an ideal diangle in the boundary of a thickened tetrahedron correspond to the two geodesics connecting the same two cusps, where two geodesics belong to the geodesic triangles that have the two cusps in common.

Let us describe a diangle $D_{0, \infty ; 1, \alpha}$ whose two vertices are 0 and $\infty$ and whose two sides are geodesics that belong to each of the ideal triangles $0,1, \infty$ and $0, \alpha, \infty$. The geodesic $l_{0}$ between the points 0 and $\infty$ that lie on the geodesic triangle $0,1, \infty$ can be parametrized in the following way: $\{(i t, i t) \mid t \in \mathbb{R}, t \geq 0\} \subset \operatorname{Im}(\mathbb{M}) \times \operatorname{Im}(\mathbb{M})$. Here by $\operatorname{Im}(\mathbb{M})$ we mean the imaginary part of the upper half-plane. The element $\gamma \in \Gamma$ that sends $0, \alpha, \infty$ to $0,1, \infty$ is $\gamma=\left(\begin{array}{cc}\alpha^{-1} & 0 \\ 0 & 1\end{array}\right)$. Then $\left(\gamma^{-1}\right)^{*}(i t, i t)=$ $\left(\left|\alpha_{1}\right| i t,\left|\alpha_{2}\right| i t\right)$. Therefore, the geodesic $l_{\alpha}$ between the points 0 and $\infty$ that lie on the geodesic triangle $0, \alpha, \infty$ can be parametrized as $\left\{\left(\left|\alpha_{1}\right| i t,\left|\alpha_{2}\right| i t\right) \mid t \in \mathbb{R}, t \geq 0\right\} \subset$ $\operatorname{Im}(\mathbb{M}) \times \operatorname{Im}(\mathbb{M})$. Then, we define the diangle $D_{0, \infty ; 1, \alpha}$ as the two-dimensional region in $\operatorname{Im}(\mathbb{H}) \times \operatorname{Im}(\mathbb{H})$ between the lines $l_{0}$ and $l_{\alpha}$. We also consider the diangle with orientation. If $\left|\alpha_{1}\right|>\left|\alpha_{2}\right|$ then it is positively oriented. If the inequality is reversed then the diangle is negatively oriented; if $\left|\alpha_{1}\right|=\left|\alpha_{2}\right|$ then it is a degenerate diangle, which consists of a single geodesic. All other diangles that we will consider are translates of $D_{0, \infty ; 1, \alpha}$ via the action of any element $\gamma \in \operatorname{PGL}_{2}(K)$.

Lemma 4.1. (i) Each geodesic triangle $\Delta_{X}$ lies either on a holomorphic curve or on an antiholomorphic curve.

(ii) Each geodesic in a geodesic triangle $\Delta_{X}$ belongs both to a holomorphic curve and to an antiholomorphic curve.

Proof. Part (i) follows from the construction of a geodesic triangle before the lemma. For part (ii), consider the following: Let $\Delta(0,1, \infty)$ be the geodesic triangle in the diagonal of $\mathbb{T}^{2}$ connecting the points 0,1 and $\infty$. It is a holomorphic curve. Thus, a geodesic $\left\{(i t, i t) \in \mathbb{U}^{2} \mid t>0\right\}$, connecting the points 0 and $\infty$ as a face of the geodesic triangle $\Delta(0,1, \infty)$, lies on a holomorphic curve. Now consider the geodesic triangle $D(0, \sqrt{d}, \infty)$. It lies on an antiholomorphic curve in $\mathbb{H}^{2}$, by which we mean a complex curve in $\mathbb{H}^{2}$ (where we have taken the complex conjugate complex structure in one of the upper half-planes), since the linear fractional transformation that sends $D(0, \sqrt{d}, \infty)$ to $D(0,1, \infty)$ does not have totally positive (or totally negative) determinant. Explicitly, the linear fractional transformation that sends $(0, \sqrt{d}, \infty)$ to $(0,1, \infty)$ is

$$
\gamma=\left(\begin{array}{cc}
1 & 0 \\
0 & \sqrt{d}
\end{array}\right)
$$

Then

$$
\left(\gamma_{1}, \gamma_{2}\right)=\left(\left(\begin{array}{cc}
1 & 0 \\
0 & \sqrt{d}
\end{array}\right),\left(\begin{array}{cc}
1 & 0 \\
0 & -\sqrt{d}
\end{array}\right)\right)
$$


We have $\gamma_{1}(i t)=(1 / \sqrt{d})$ it and $\gamma_{2}(i t)=-(1 / \sqrt{d}) \overline{i t}=\gamma_{1}(i t)$. Then the same geodesic $(i t, i t)$ belongs to the antiholomorphic curve given by the pullback of the diagonal with respect to the linear fractional map $\gamma$. Thus, we obtain that the geodesic (it, it) connecting 0 and $\infty$ belongs to both a holomorphic curve and an antiholomorphic curve. Similarly, any translate of the geodesic (it, it) via a linear fractional map from $\mathrm{GL}_{2}(K)$ would belong to both a holomorphic curve and an antiholomorphic curve. That proves part (ii).

Definition 4.2. Let $p_{1}, p_{2}, p_{3}, p_{4}$ be cusp points in $\mathbb{M}^{2} \cup \mathbb{P}^{1}(K)$. To each triple of points $p_{1}, p_{2}, p_{3}$, we associate the geodesic triangle $\left\{p_{1}, p_{2}, p_{3}\right\}$ with coefficient 1 as an element of the singular chain complex in $C_{2}\left(\mathbb{H}^{2} \cup \mathbb{P}^{1}(K), \mathbb{Q}\right)$. Also, to each quadruple of points $p_{1}, p_{2}, p_{3}, p_{4}$, we associate the geodesic diangle between the two geodesic connecting $p_{1}$ and $p_{2}$ so that the first geodesic is a face of the geodesic triangle $\left\{p_{1}, p_{2}, p_{3}\right\}$ and the second geodesic is a face of the geodesic triangle $\left\{p_{1}, p_{2}, p_{4}\right\}$. We denote such a diangle by $\left\{p_{1}, p_{2} ; p_{3}, p_{4}\right\}$. We call the geodesic triangle $\left\{p_{1}, p_{2}, p_{3}\right\}$ and the geodesic diangle $\left\{p_{1}, p_{2} ; p_{3}, p_{4}\right\}$, considered as elements of $C_{2}\left(\mathbb{M}^{2} \cup \mathbb{P}^{1}(K), \mathbb{Q}\right)$, commutative Hilbert modular symbols.

Theorem 4.3. The commutative Hilbert modular symbols, modulo the boundary of singular 3-chains $\partial C_{3}\left(\mathbb{H}^{2} \cup \mathbb{P}^{1}(K), \mathbb{Q}\right)$, satisfy the following properties:

(1) If $\sigma$ is a permutation of the set $\{1,2,3\}$ then

$$
\left\{p_{\sigma(1)}, p_{\sigma(2)}, p_{\sigma(3)}\right\}=\operatorname{sign}(\sigma)\left\{p_{1}, p_{2}, p_{3}\right\}
$$

(2) If $p_{1}, p_{2}, p_{3}, p_{4}$ are four points on the same holomorphic (or antiholomorphic) curve of the type $\gamma^{*} \Delta$, then

$$
\left\{p_{1}, p_{2}, p_{3}\right\}+\left\{p_{2}, p_{3}, p_{4}\right\}=\left\{p_{1}, p_{2}, p_{4}\right\}+\left\{p_{1}, p_{3}, p_{4}\right\}
$$

For every four points $p_{1}, p_{2}, p_{3}, p_{4}$, we associate a diangle with vertices $p_{1}$ and $p_{2}$. Let $\left\{p_{1}, p_{2} ; p_{3}, p_{4}\right\}$ be the corresponding symbol.

(3) If $p_{1}, p_{2}, p_{3}, p_{4}$ are four points on the same holomorphic (or antiholomorphic) curve of type $\gamma^{*} \Delta$, then

$$
0=\left\{p_{1}, p_{2} ; p_{3}, p_{4}\right\}
$$

(4) For every distinct four points $p_{1}, p_{2}, p_{3}, p_{4}$, we have the following relations, based on the orientation of the domain:

$\left\{p_{2}, p_{1} ; p_{3}, p_{4}\right\}=\left\{p_{1}, p_{2} ; p_{4}, p_{3}\right\}=-\left\{p_{2}, p_{1} ; p_{4}, p_{3}\right\}=-\left\{p_{1}, p_{2} ; p_{3}, p_{4}\right\}$. 
(5) For every five points $p_{1}, p_{2}, p_{3}, p_{4}, p_{5}$, we have

$$
\left\{p_{1}, p_{2} ; p_{3}, p_{4}\right\}+\left\{p_{1}, p_{2} ; p_{4}, p_{5}\right\}=\left\{p_{1}, p_{2} ; p_{3}, p_{5}\right\} .
$$

(6) We also have a relation between the two types of commutative Hilbert modular symbols. For every four distinct points $p_{1}, p_{2}, p_{3}, p_{4}$, we have

$$
\begin{aligned}
0= & \left\{p_{1}, p_{2}, p_{3}\right\}+\left\{p_{2}, p_{3}, p_{4}\right\}-\left\{p_{1}, p_{2}, p_{4}\right\}-\left\{p_{1}, p_{3}, p_{4}\right\} \\
& +\left\{p_{1}, p_{2} ; p_{3}, p_{4}\right\}+\left\{p_{2}, p_{3} ; p_{1}, p_{4}\right\}+\left\{p_{3}, p_{1} ; p_{2}, p_{4}\right\} \\
& +\left\{p_{3}, p_{4} ; p_{1}, p_{2}\right\}+\left\{p_{1}, p_{4} ; p_{2}, p_{3}\right\}+\left\{p_{2}, p_{4} ; p_{3}, p_{1}\right\}
\end{aligned}
$$

Proof. Part (1) follows from the orientation of the simplex in singular homology. Part (2) is an equality induced by two different triangulations on a holomorphic (or antiholomorphic) curve with four vertices. In that setting the diangles are trivial, which proves part (3). Part (4) follows from the orientation of the diangle. Part (5) corresponds to a union of two geodesic diangles with a common face, given by a third geodesic diangle. Part (5) will be used for a noncommutative 1-cocycle relation for the noncommutative Hilbert modular symbol (see Conjecture 4.14). Part (6) is a boundary relation for the boundary of a thickened tetrahedron. By a thickened tetrahedron, we mean a union of four geodesic triangles corresponding to each triple of points among the four points $p_{1}, p_{2}, p_{3}, p_{4}$ together with six geodesic diangles that correspond to the area between the faces of the geodesic triangles. They correspond exactly to the thickening of the six edges of a tetrahedron.

Part 6 will be used to derive explicit formulas for the noncommutative Hilbert modular symbol of type $\boldsymbol{c}^{\prime}$ resembling a noncommutative 2-cocycle relation (see Conjecture 4.15).

4B. Pairing of the modular symbols with cohomology. In this subsection, we consider pairings between commutative Hilbert modular symbols and cusp forms. In some cases, we prove that such pairings give periods in the sense of [Kontsevich and Zagier 2001].

We are interested in holomorphic cusp forms with respect to $\Gamma$. Equivalently, we can consider the holomorphic 2-forms on $\widetilde{X}$, the minimal smooth algebraic compactification of $X$ [Hirzebruch 1973]. At this point we should distinguish between geodesic triangles that lie on a holomorphic curve and those that lie on an antiholomorphic curve. The reason for this distinction is that a holomorphic 2-form restricted to a holomorphic curve vanishes. The way to distinguish the two types of geodesic triangles is the following: Let $\gamma$ be a linear fractional transform that sends the points $p_{1}, p_{2}, p_{3}$ to $0,1, \infty$. If $\operatorname{det} \gamma$ is totally positive or totally negative, then the geodesic triangle $p_{1}, p_{2}, p_{3}$ lies on a holomorphic curve. If $\operatorname{det} \gamma$ is neither totally positive nor totally negative, then the geodesic triangle $p_{1}, p_{2}, p_{3}$ lies on an antiholomorphic curve. 
Definition 4.4. Define $M_{2}\left(\mathbb{M}^{2} \cup \mathbb{P}^{1}(K), \mathbb{Q}\right)$ to be the span of the Hilbert modular symbols $\left\{p_{1}, p_{2}, p_{3}\right\}$ and $\left\{p_{1}, p_{2} ; p_{3}, p_{4}\right\}$ as a subspace of the singular chain $C_{2}\left(\mathbb{M}^{2} \cup \mathbb{P}^{1}(K), \mathbb{Q}\right)$. We define the pairing

$$
\langle,\rangle: M_{2}\left(\mathbb{H}^{2} \cup \mathbb{P}^{1}(K)\right) \times S_{2,2}(\Gamma) \rightarrow \mathbb{C}
$$

by setting

$$
\left\langle\left\{p_{1}, p_{2}, p_{3}\right\}, f d z_{1} \wedge d z_{2}\right\rangle=\int_{\left\{p_{1}, p_{2}, p_{3}\right\}} f d z_{1} \wedge d z_{2}
$$

for geodesic triangles and

$$
\left\langle\left\{p_{1}, p_{2} ; p_{3}, p_{4}\right\}, f d z_{1} \wedge d z_{2}\right\rangle=\int_{\left\{p_{1}, p_{2} ; p_{3}, p_{4}\right\}} f d z_{1} \wedge d z_{2}
$$

for geodesic diangles.

We are going to use that a Hilbert modular surface $X(\mathbb{C})$ can be realized as the complex points of an arithmetic surface defined over a number field $F$.

Theorem 4.5. The image of the above pairing is a period over a number field $F$ when we integrate a normalized cusp Hecke eigenform $f$ of weight $(2,2)$. (For Hecke eigenforms, see [Shimura 1978; Berger et al. 2013].)

Proof. From Lemma 3.1(ii), the boundary of the geodesic triangles of the diangles are geodesics that lie on holomorphic curves in $\mathbb{T}^{2} \cup \mathbb{P}^{1}(K)$. Therefore, in the quotient by the congruence group $\Gamma$, the geodesic lies in a Hirzebruch-Zagier divisor on the Hilbert modular surface. Thus, we integrate a closed algebraic differential 2-form (that is, a global differential 2-form with algebraic coefficients) on the Hilbert modular surface, with boundaries Hirzebruch-Zagier divisors.

Conjecture 4.6. Let $f \in S_{k, k}(\Gamma)$ be a normalized cusp Hecke eigenform of weight $(k, k)$. Then

$$
\int_{\left\{p_{1}, p_{2}, p_{3}\right\}} f d z_{1} \wedge d z_{2}
$$

for geodesic triangles and

$$
\int_{\left\{p_{1}, p_{2} ; p_{3}, p_{4}\right\}} f d z_{1} \wedge d z_{2}
$$

for geodesic diangles are periods.

Theorem 4.5 is a proof of Conjecture 4.6 for the case of cusp form of weight $(2,2)$. 
4C. Iteration - revisited. We defined iterated integrals on diangles in Definitions 3.14 and 3.15. However, these definitions have to be extended to other domains of integration in order to consider iterated integrals on geodesic triangles.

A consequence of the results from this subsection is the following:

Theorem 4.7. Iterated integrals of type $c$ on a geodesic diangle and on a geodesic triangle of algebraic differential 2-forms on a Hilbert modular surface are periods in the sense of Kontsevich-Zagier.

Before giving the proof, we need definitions of several objects, as well as their properties. In the process, we will be able to extend the definition of iterated integrals on membranes when the domain of integration is a geodesic triangle.

For type $\boldsymbol{b}$, in Definition 3.14, we have a map $g: U \rightarrow \mathbb{U}^{2}$ that sends the two $\mathbb{R}$-foliations on $U$ into two coordinatewise $\mathbb{C}$-foliations of $\mathbb{H}^{2}$. The same definition does not work when the domain $U$ is a geodesic triangle. The reason is that a geodesic triangle is either a holomorphic curve or an antiholomorphic curve. In both cases, a pullback of one leaf to the geodesic triangle is a point not a line (which is the case for the diangles).

In order to extend Definitions 3.14 and 3.15 to the case when the domain $U$ is a geodesic triangle, we are going to construct a new space using the fiber products multiple times.

Now, we are going to define a space $Y_{n}$ associated to an iterated integral on $n$ 2 -forms on $\mathbb{H}^{2}$. We are going to use fiber products (see [Hartshorne 1977]). Let $p_{1}$ and $p_{2}$ be the projections of $\mathbb{H}^{2}$ on the first and the second component, respectively. Define $X_{i j}=\mathbb{H}^{2}$ for $1 \leq i \leq n$ and $1 \leq j \leq n$. (One should think of the component $X_{i j}$ as the complexification of the real coordinate $\left(s_{i}, t_{j}\right)$.) Let $C_{i}=\mathbb{U}$ for $1 \leq i \leq n$ and $C_{j}^{\prime}=\mathbb{H}$ for $1 \leq j \leq n$. Let

$$
X_{j}=X_{1 j} \times_{C_{j}^{\prime}} X_{2 j} \times_{C_{j}^{\prime}} \cdots \times_{C_{j}^{\prime}} X_{n j} .
$$

$\left(X_{j}\right.$ corresponds to the variable $t_{j}$.) Then

$$
X_{j} \subset X_{1 j} \times X_{2 j} \times \cdots \times X_{n j}
$$

Let also

$$
P_{j}=\left(p_{1}, \ldots, p_{1}\right): X_{1 j} \times X_{2 j} \times \cdots \times X_{n j} \rightarrow C_{1} \times \cdots \times C_{n} .
$$

Let $P_{j}^{\circ}=\left.P_{j}\right|_{X_{j}}$ be the restriction of $P_{j}$ to the subset $X_{j}$. We define $Y_{n}$ as the fiber product of $X_{1}, \ldots, X_{n}$ with respect to the morphisms $P_{1}^{\circ}, \ldots, P_{n}^{\circ}$ over the base $C_{1} \times \cdots \times C_{n}$, namely

$$
Y_{n}=X_{1} \times{ }_{C} \cdots \times_{C} X_{n},
$$

where $C=C_{1} \times \cdots \times C_{n}$. Note that $X_{j}$ is isomorphic to $X_{j+1}$. Let $Z_{j}$ be the subspace of $Y_{n}$ defined by setting the $j$ - and the $(j+1)$-components of $Y_{n}=X_{1} \times{ }_{C} \cdots \times{ }_{C} X_{n}$ 
to be equal. (The space $Z_{j}$ corresponds to a boundary components obtained by letting $t_{j}=t_{j+1}$.) Similarly, we could have defined $Y_{n}$ by defining first

$$
X_{i}^{\prime}=X_{i 1} \times{ }_{C_{i}} X_{i 2} \times{ }_{C_{i}} \cdots \times{ }_{C_{i}} X_{i n}
$$

$\left(X_{i}^{\prime}\right.$ corresponds to $\left.s_{i}\right)$ so that

$$
X_{i}^{\prime} \subset X_{i 1} \times X_{i 2} \times \cdots \times X_{i n}
$$

Let

$$
P_{i}^{\prime}=\left(p_{2}, \ldots, p_{2}\right): X_{i 1} \times X_{i 2} \times \cdots \times X_{i n} \rightarrow C_{1}^{\prime} \times \cdots \times C_{n}^{\prime} .
$$

Define $P_{i}^{\prime \circ}=\left.P_{i}^{\prime}\right|_{X_{i}^{\prime}}$ to be the restriction of $P_{i}^{\prime}$ to $X_{i}^{\prime}$. We define $Y_{n}$ as the fiber product of $X_{1}^{\prime}, \ldots, X_{n}^{\prime}$ with respect to the morphisms $P_{1}^{\prime \circ}, \ldots, P_{n}^{\prime \circ}$ over the base $C_{1}^{\prime} \times \cdots \times C_{n}^{\prime}$, namely,

$$
Y_{n}=X_{1}^{\prime} \times{ }_{C^{\prime}} \cdots \times \times_{C^{\prime}} X_{n}^{\prime},
$$

where $C^{\prime}=C_{1}^{\prime} \times \cdots \times C_{n}^{\prime}$. Similarly we define $Z_{i}^{\prime}$ to be the subspace of $Y_{n}$ defined by setting the $i$ - and the $(i+1)$-components of $Y_{n}=X_{1}^{\prime} \times{ }_{C^{\prime}} \cdots \times{ }_{C^{\prime}} X_{n}^{\prime}$ to be equal. (The space $Z_{i}^{\prime}$ corresponds to a boundary components obtained by letting $s_{i}=s_{i+1}$.)

We have given two definitions (22) and (23) of the space $Y_{n}$. In the two definitions we have only exchanged the role of $p_{1}$ and $p_{2}$. We will prove that both definitions lead to the same object in the case $n=2$. The general case is left to the reader.

Lemma 4.8. For $n=2$, the two definitions (22) and (23) define isomorphic objects $Y_{2}$.

Proof. The space $Y_{2}$ can be defined as a finite limit (in a categorical sense) of a diagram in the following way. Consider the commutative diagram

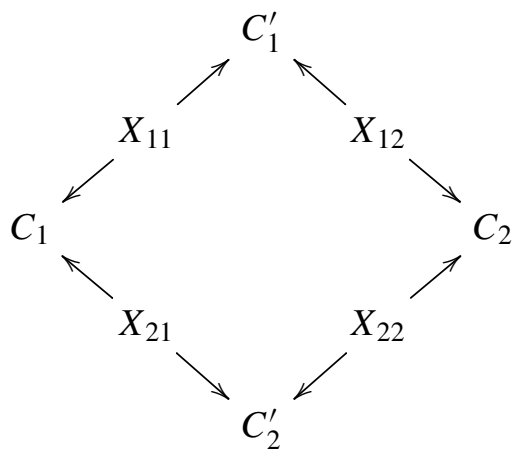


For any space $W$ such that

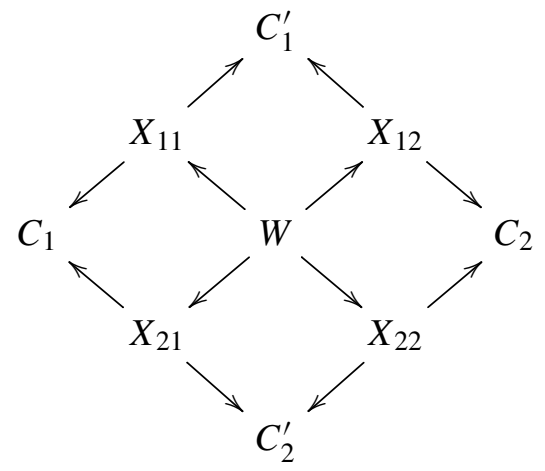

commutes, we have that the maps $f_{i j}: W \rightarrow X_{i j}$ factor through $g_{i j}: Y_{2} \rightarrow X_{i j}$, so that $f_{i j}=g_{i j} \circ h$ for some $h: W \rightarrow Y_{2}$, and $Y_{2}$ is part of the commutative diagram

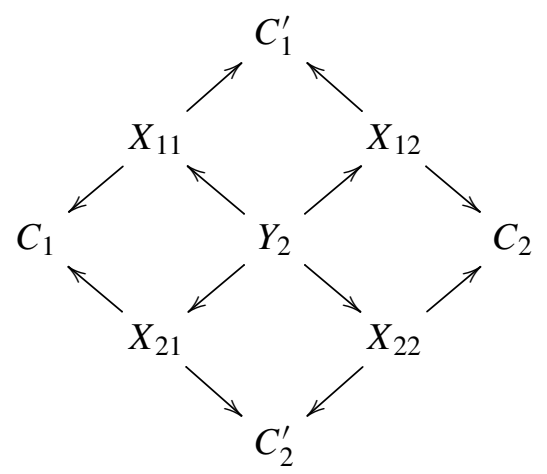

In order to prove this universal property of $Y_{2}$ we follow the first definition of $Y_{2}$. This leads to the commutative diagram

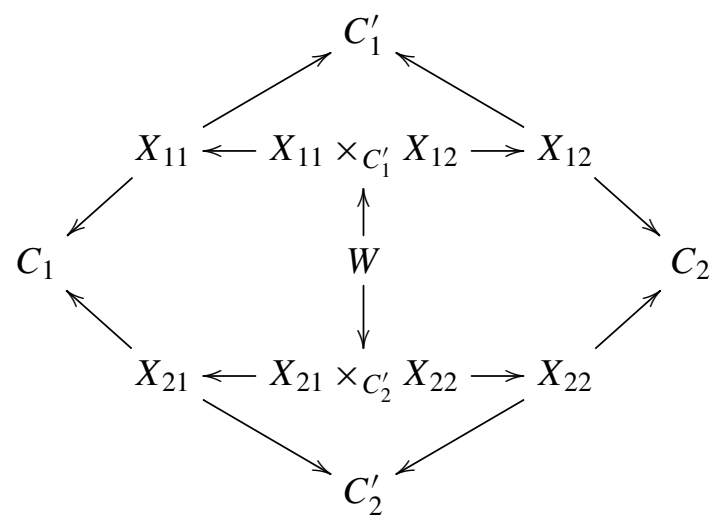

Then we have that $X_{1}=X_{11} \times{ }_{C_{1}^{\prime}} X_{12}$ maps to $C=C_{1} \times C_{2}$ and also $X_{2}=X_{21} \times{ }_{C_{2}^{\prime}} X_{22}$ maps to $C=C_{1} \times C_{2}$. Thus the maps from $W$ to any element of the diagram 
factors through $Y_{2}=X_{1} \times_{C} X_{2}$. Similarly, $W$ factors through $X_{1}^{\prime} \times{ }_{C^{\prime}} X_{2}^{\prime}$, where $X_{1}^{\prime}=X_{11} \times_{C_{1}} X_{21}, X_{2}^{\prime}=X_{12} \times_{C_{2}} X_{22}$ and $C^{\prime}=C_{1}^{\prime} \times C_{2}^{\prime}$. Since both $X_{1} \times_{C} X_{2}$ and $X_{1}^{\prime} \times{ }_{C^{\prime}} X_{2}^{\prime}$ are universal objects with respect to the diagram (24), we have that they are isomorphic.

Now, we return to the initial question of this subsection, namely, how to iterate over a geodesic triangle so that it is consistent with the current definition of iteration over a diangle.

For an $n$-fold iteration of 2 -forms of types $\boldsymbol{b}$ or $\boldsymbol{c}$, we have to specify a domain $U \subset \mathbb{H}^{2}, \operatorname{dim}_{\mathbb{R}} U=2$, and a pair of permutations $\rho_{1}$ and $\rho_{2}$ of $n$ elements. We make an essential assumption that the boundary of $U \subset \mathbb{H}^{2}$, denoted by $\partial U$, projected onto the Hilbert modular surface $Y_{\Gamma}$ lies on a finite union of Hirzebruch-Zagier divisors. We will denote the finite union of such Hirzebruch-Zagier divisors by $\mathrm{HZ}$.

Let

$$
P_{\rho_{1}, \rho_{2}}: X_{11} \times \cdots \times X_{n n} \rightarrow X_{\rho_{1}(1) \rho_{2}(1)} \times \cdots \times X_{\rho_{1}(n) \rho_{2}(n)}
$$

be a projection to $n$ of the factors. Let $U_{i j} \cong U$ for $1 \leq i \leq n$ and $1 \leq j \leq n$. Let

$$
I: U_{\rho_{1}(1) \rho_{2}(1)} \times \cdots \times U_{\rho_{1}(n) \rho_{2}(n)} \rightarrow X_{\rho_{1}(1) \rho_{2}(1)} \times \cdots \times X_{\rho_{1}(n) \rho_{2}(n)}
$$

be induced from the product of inclusion of the domains $U \rightarrow X$. We will use the notation

$$
\underline{U}^{\rho}=U_{\rho_{1}(1) \rho_{2}(1)} \times \cdots \times U_{\rho_{1}(n) \rho_{2}(n)}
$$

and

$$
\underline{X}^{\rho}=X_{\rho_{1}(1) \rho_{2}(1)} \times \cdots \times X_{\rho_{1}(n) \rho_{2}(n)} .
$$

Then the map I becomes

$$
I: \underline{U}^{\rho} \rightarrow \underline{X}^{\rho}
$$

Let

$$
J: Y_{n} \rightarrow \underline{X}^{\rho}
$$

be the composition of the natural inclusion $Y_{n} \rightarrow X_{11} \times \cdots \times X_{n n}$ and the projection $P_{\rho_{1}, \rho_{2}}$. Then we define the domain of integration to be

$$
\underline{U}_{Y_{n}}^{\rho}=\underline{U}^{\rho} \times_{\underline{X}^{\rho}} Y_{n},
$$

which is the fiber product of the maps $I$ and $J$. Since $I: \underline{U}^{\rho} \rightarrow \underline{X}^{\rho}$ is an inclusion, we have that the induced map

$$
\underline{U}_{Y_{n}}^{\rho} \rightarrow Y_{n}
$$

is an inclusion.

In the above constructions, we have used a parallel between type $\boldsymbol{b}$ and type $\boldsymbol{c}$ iterated integrals on membranes. The following definition allows us to extend in some sense the two types when the domain of integration is an ideal triangle: 
Definition 4.9 (iterated integrals on membranes of types $\boldsymbol{b}^{\prime}$ or $\boldsymbol{c}^{\prime}$ ). For any manifold with corners of dimension 2 on a Hilbert modular variety, we define an iterated integral

$$
\begin{aligned}
\int_{U}^{\Sigma_{n}\left(\rho_{1}, \rho_{2}\right)}\left(f_{1} d z_{1} \wedge d z_{2}\right) \cdots( & \left.f_{n} d z_{1} \wedge d z_{2}\right) \\
& =\int_{\underline{U}_{Y_{n}}^{\rho}} J^{*}\left(f_{1} d z_{1} \wedge d z_{2}, \ldots, f_{n} d z_{1} \wedge d z_{2}\right),
\end{aligned}
$$

where $f_{k} d z_{1} \wedge d z_{2}$ is a form defined on $X_{\rho_{1}(k) \rho_{2}(k)}$ for $1 \leq k \leq n$. If $Y_{n}$ and $\underline{U}_{Y_{n}}^{\rho}$ are constructed in the setting of type $\boldsymbol{b}$ iterated integrals on membranes, then the above definition is of iterated integrals on membranes of type $\boldsymbol{b}^{\prime}$. Similarly, if $Y_{n}$ and $\underline{U}_{Y_{n}}^{\rho}$ are constructed in the setting of type $c$ iterated integrals on membranes, then the above definition is of iterated integrals on membranes of type $\boldsymbol{c}^{\prime}$.

If $U$ is a diangle, then the relation of the above integral to the ones defined by iterated integrals over membranes is the following: The integral

$$
\int_{U}^{\Sigma_{n}\left(\rho_{1}, \rho_{2}\right)}\left(f_{1} d z_{1} \wedge d z_{2}\right) \cdots\left(f_{n} d z_{1} \wedge d z_{2}\right)
$$

is the sum of the integrals from Definitions 3.14 or 3.15, namely, the sum

$$
\sum_{\rho \in \Sigma_{n}} \int_{U}^{\left(\rho \rho_{1}, \rho \rho_{2}\right)}\left(f_{1} d z_{1} \wedge d z_{2}\right) \cdots\left(f_{n} d z_{1} \wedge d z_{2}\right)
$$

over the orbit of the diagonal action of the permutation group $\Sigma_{n}$ on any chosen pair of permutations $\left(\rho_{1}, \rho_{2}\right)$.

Proposition 4.10 (properties of the iterated integral (25)). (1) The iterated integral (25) is well-defined when $U$ is an ideal triangle both for types $\boldsymbol{b}$ and $\boldsymbol{c}$.

(2) The iterated integral (25) for type $c$ is a period if $U$ is an ideal triangle or an ideal diangle, when $f_{1}, \ldots, f_{n}$ are normalized Hecke eigenforms of weight $(2,2)$.

(3) The iterated integral (25), both for types $\boldsymbol{b}$ and $\boldsymbol{c}$, is homotopy invariant with respect to homotopies that vary within the divisors

$$
J^{-1}\left(X_{\rho_{1}(1) \rho_{2}(1)} \times \cdots \times p_{1}^{-1}\left(q_{i}\right) \times \cdots \times X_{\rho_{1}(n) \rho_{2}(n)}\right),
$$

where $q_{i}$ is a point of $X_{\rho_{1}(i) \rho_{2}(i)}$ for fixed $i$ and $p_{1}: X_{\rho_{1}(i) \rho_{2}(i)} \rightarrow C$; or homotopies that vary within the divisors

$$
J^{-1}\left(X_{\rho_{1}(1) \rho_{2}(1)} \times \cdots \times p_{2}^{-1}\left(q_{i}\right) \times \cdots \times X_{\rho_{1}(n) \rho_{2}(n)}\right),
$$

where $q_{i}$ is a point of $X_{\rho_{1}(i) \rho_{2}(i)}$ for fixed $i$ and $p_{2}: X_{\rho_{1}(i) \rho_{2}(i)} \rightarrow C^{\prime}$. 
Proof. (a) The integral (25) is well-defined for any two-dimensional submanifold with corners of the Hilbert modular variety [Borel and Serre 1973].

(b) The iterated integral (25) is a period since:

(1) A Hilbert modular variety can be defined over a number field.

(2) The normalized Hecke eigenforms $f_{1}, \ldots, f_{n}$ of weight $(2,2)$ can be realized as algebraic differential forms on the Hilbert modular variety.

(3) The boundary of the region of integration $\bar{U}_{Y_{n}}^{\rho}$ is a divisor on $Y_{n}$, namely,

$$
\bigcup_{i=1}^{n} \mathrm{HZ}_{i} \text {, }
$$

where

$$
\mathrm{HZ}_{i}=J^{-1}\left(X_{\rho_{1}(1) \rho_{2}(1)} \times \cdots \times \mathrm{HZ} \times \cdots \times X_{\rho_{1}(n) \rho_{2}(n)}\right)
$$

is a divisor of $Y_{n}$ obtained as a pullback of a divisor whose $i$-th component is a Hirzebruch-Zagier divisor HZ, and the rest of the factors are $X_{\rho_{1}(k) \rho_{2}(k)}$ for $k \neq i$.

(c) The proof is essentially the same as that of Theorem 3.7.

The domain $\underline{U}_{Y_{n}}$ might be cut into disconnected components by the $Z_{i}$ and $Z_{j}^{\prime}$. In order to choose a connected component we need to define another region of integration. Recall that for the case of iterated integrals on membranes of type $\boldsymbol{b}, p_{1}: \mathbb{H}^{2} \rightarrow C$ and $p_{2}: \mathbb{H}^{2} \rightarrow C^{\prime}$ are projections onto the first and the second component, with $C \cong \mathbb{U}$ and $C^{\prime} \cong \mathbb{H}$.

For the case of iterated integrals on membranes of type $\boldsymbol{c}, p_{1}=\alpha_{1} \circ \pi$ and $p_{2}=\alpha_{2} \circ \pi$ are compositions of

$$
\pi: \mathbb{W}^{2} \rightarrow X_{\Gamma},
$$

the map from the universal cover to the Hilbert modular surface, with

$$
\alpha_{1}, \alpha_{2}: X_{\Gamma} \rightarrow \mathbb{P}^{1}
$$

two algebraically independent rational functions on the Hilbert modular surface, and $C_{i} \cong \mathbb{P}^{1}$ and $C_{j}^{\prime} \cong \mathbb{P}^{1}$ for $1 \leq i \leq n$ and $1 \leq j \leq n$.

Let $q_{0}, q_{1}, r_{0}, r_{1} \in \mathbb{P}^{1}$ be points. Let $Q_{0}, Q_{1}, R_{0}$ and $R_{1}$ be connected components of $p_{1}^{-1}\left(q_{0}\right), p_{1}^{-1}\left(q_{1}\right), p_{2}^{-1}\left(r_{0}\right)$, and $p_{2}^{-1}\left(r_{1}\right)$, respectively.

Let $V \rightarrow \mathbb{H}^{2}$ be a domain in $\mathbb{T}^{2}$ with boundary on the union

$$
Q_{0} \cup Q_{1} \cup R_{0} \cup R_{1},
$$

but with interior disjoint from this union. We define the divisors $Z_{0}, Z_{n}, Z_{0}^{\prime}, Z_{n}^{\prime}$ of $Y_{n}$ as follows: $Z_{0}$ will be the beginning of the integration of the $t_{1}$ variable $\left(t_{1}=0\right)$, 
$Z_{n}$ will be the end of the integration of the $t_{n}$ variable $\left(t_{n}=1\right), Z_{0}^{\prime}$ will be the beginning of the integration of the $s_{1}$ variable $\left(s_{1}=0\right)$, and $Z_{n}^{\prime}$ will be the end of the integration of the $s_{n}$ variable $\left(s_{n}=1\right)$. We define them as the fiber product

$$
\begin{array}{ll}
Z_{0}=Q_{0} \times_{C} X_{2} \times_{C} \cdots \times_{C} X_{n}, & Z_{n}=X_{1} \times_{C} \cdots \times_{C} X_{n-1} \times_{C} Q_{1}, \\
Z_{0}^{\prime}=R_{0} \times_{C^{\prime}} X_{2}^{\prime} \times_{C^{\prime}} \cdots \times_{C^{\prime}} X_{n}^{\prime}, & Z_{n}^{\prime}=X_{1}^{\prime} \times_{C^{\prime}} \cdots \times_{C^{\prime}} X_{n-1}^{\prime} \times_{C^{\prime}} R_{1} .
\end{array}
$$

We will use the notation

$$
\underline{V}^{\rho}=V_{\rho_{1}(1) \rho_{2}(1)} \times \cdots \times V_{\rho_{1}(n) \rho_{2}(n)} \quad \text { and } \quad \underline{X}^{\rho}=X_{\rho_{1}(1) \rho_{2}(1)} \times \cdots \times X_{\rho_{1}(n) \rho_{2}(n)} .
$$

Then the map $I^{\prime}$ becomes

$$
I^{\prime}: \underline{V}^{\rho} \rightarrow \underline{X}^{\rho}
$$

Let

$$
J^{\prime}: Y_{n} \rightarrow \underline{X}^{\rho}
$$

be the composition of the natural inclusion $Y_{n} \rightarrow X_{11} \times \cdots \times X_{n n}$ and the projection $P_{\rho_{1}, \rho_{2}}$. Then we define the domain of integration to be

$$
\underline{V}_{Y_{n}}^{\rho}=\underline{V}^{\rho} \times_{\underline{X}^{\rho}} Y_{n},
$$

which is the fiber product of the maps $I^{\prime}$ and $J^{\prime}$. Since $I^{\prime}: \underline{U}^{\rho} \rightarrow \underline{X}^{\rho}$ is an inclusion, we have that the induced map

$$
\underline{V}_{Y_{n}}^{\rho} \rightarrow Y_{n}
$$

is an inclusion.

Then the divisors $Z_{0}, Z_{1}, \ldots, Z_{n-1}, Z_{n}$ and $Z_{0}^{\prime}, Z_{1}^{\prime}, \ldots, Z_{n-1}^{\prime}, Z_{n}^{\prime}$ cut out from $V_{Y_{n}}$ a product of two $n$-simplices, which corresponds to the region where the product $\left\{0 \leq s_{1} \leq \cdots \leq s_{n} \leq 1\right\} \times\left\{0 \leq t_{1} \leq \cdots \leq t_{n} \leq 1\right\}$ is embedded. Denote by $\bar{V}_{Y_{n}}^{\rho}$ the connected components of $\bar{V}_{Y_{n}}^{\rho}$ that contains the image of the product $\left\{0 \leq s_{1} \leq \cdots \leq s_{n} \leq 1\right\} \times\left\{0 \leq t_{1} \leq \cdots \leq t_{n} \leq 1\right\}$ under the map $g$ from Definition 3.5.

Proof of Theorem 4.7. We consider the type of iterated integrals defined in Definition 3.15. Using the above notation, the domain of integration is $U \subset V$. We define

$$
\bar{U}_{Y_{n}}^{\rho}=\underline{U}_{Y_{n}}^{\rho} \cap \bar{V}_{Y_{n}}^{\rho} .
$$

Then the boundary of $\bar{U}_{Y_{n}}^{\rho}$ lies on the union of divisors

$$
\partial \bar{U}_{Y_{n}}^{\rho} \subset\left(\bigcup_{i=1}^{n} Z_{i}\right) \cup\left(\bigcup_{j=1}^{n} Z_{j}^{\prime}\right) .
$$

The normalized Hecke eigenforms of weight $(2,2)$ can be realized as algebraic differential forms on the Hilbert modular variety. Then the iterated integrals on a membrane of type $c$ over the domain $U$ are periods, since: 
(1) A Hilbert modular variety can be defined over a number field.

(2) The normalized Hecke eigenforms $f_{1}, \ldots, f_{n}$ of weight $(2,2)$ can be realized as algebraic differential forms on the Hilbert modular variety.

(3) The boundary of the region of integration $\bar{U}_{Y_{n}}^{\rho}$ is a divisor on $Y_{n}$, namely,

$$
\left(\bigcup_{i=1}^{n} Z_{i}\right) \cup\left(\bigcup_{j=1}^{n} Z_{j}^{\prime}\right) \text {. }
$$

4D. Generating series and relations. In this subsection, we examine the generating series of iterated integrals on membranes (of types $\boldsymbol{b}^{\prime}$ or $\boldsymbol{c}^{\prime}$ ), evaluated at geodesic triangles and geodesic diangles. We prove relations among them. Most importantly, the generating series $J$ will be used in Section 4E to define noncommutative Hilbert modular symbols. Moreover, the relations that we prove in this subsection will be interpreted as cocycles or as coboundaries of the noncommutative Hilbert modular symbols satisfy in Section 4E.

Definition 4.11. Let $f_{1}, \ldots, f_{m}$ be $m$ cusp forms with respect to a Hilbert modular group $\Gamma$. Let $f_{1} d z_{1} \wedge d z_{2}, \ldots, f_{m} d z_{1} \wedge d z_{2}$ be the corresponding differential forms defining the generating series. Let $J\left(p_{1}, p_{2}, p_{3}\right)$ be the generating series $J$ evaluated at the geodesic triangle with vertices $p_{1}, p_{2}, p_{3}$. Let $J\left(p_{1}, p_{2} ; p_{3}, p_{4}\right)$ be the generating series $J$ evaluated at the geodesic diangle $\left\{p_{1}, p_{2} ; p_{3}, p_{4}\right\}$.

Both $J\left(p_{1}, p_{2}, p_{3}\right)$ and $J\left(p_{1}, p_{2} ; p_{3}, p_{4}\right)$ will be called noncommutative Hilbert modular symbols after the action of the arithmetic group is included (see Definition 4.13).

Theorem 4.12. The generating series $J$ is one of the types $\boldsymbol{b}, \boldsymbol{c}, \boldsymbol{b}^{\prime}$ or $\boldsymbol{c}^{\prime}$. Note that $J\left(p_{1}, p_{2} ; p_{3}, p_{4}\right)$ is defined for all types, while $J\left(p_{1}, p_{2}, p_{3}\right)$ is defined only for types $\boldsymbol{b}^{\prime}$ or $\boldsymbol{c}^{\prime}$. Then the generating series $J\left(p_{1}, p_{2}, p_{3}\right)$ and $J\left(p_{1}, p_{2} ; p_{3}, p_{4}\right)$ satisfy the following relations:

(1) If $\sigma$ is a permutation of the set $\{1,2,3\}$, then

$$
J\left(p_{\sigma(1)}, p_{\sigma(2)}, p_{\sigma(3)}\right)=J^{\operatorname{sign}(\sigma)}\left(p_{1}, p_{2}, p_{3}\right) .
$$

(2) If $p_{1}, p_{2}, p_{3}, p_{4}$ are four points on the same holomorphic (or antiholomorphic) curve of type $\gamma^{*} \Delta$, then

$$
\begin{aligned}
1= & J\left(p_{1}, p_{2}, p_{3}\right) J\left(p_{2}, p_{3}, p_{4}\right) \\
& \times J\left(p_{2}, p_{1}, p_{4}\right) J\left(p_{1}, p_{4}, p_{3}\right) .
\end{aligned}
$$

(3) If $p_{1}, p_{2}, p_{3}, p_{4}$ are four points on the same holomorphic (or antiholomorphic) curve of type $\gamma^{*} \Delta$, then

$$
1=J\left(p_{1}, p_{2} ; p_{3}, p_{4}\right)
$$


(4) For every four points $p_{1}, p_{2}, p_{3}, p_{4}$, we have the following relation based on the orientation of the domain:

$$
\begin{aligned}
J\left(p_{2}, p_{1} ; p_{3}, p_{4}\right) & =J\left(p_{1}, p_{2} ; p_{4}, p_{3}\right) \\
& =J^{-1}\left(p_{2}, p_{1} ; p_{4}, p_{3}\right) \\
& =J^{-1}\left(p_{1}, p_{2} ; p_{3}, p_{4}\right) .
\end{aligned}
$$

(5) For every five points $p_{1}, p_{2}, p_{3}, p_{4}, p_{5}$, we have

$$
J\left(p_{1}, p_{2} ; p_{3}, p_{4}\right) J\left(p_{1}, p_{2} ; p_{4}, p_{5}\right)=J\left(p_{1}, p_{2} ; p_{3}, p_{5}\right) .
$$

(6) For every four points $p_{1}, p_{2}, p_{3}, p_{4}$, we have the following relation, based on the boundary of a thickened tetrahedron:

$$
\begin{aligned}
1= & J\left(p_{1}, p_{2}, p_{3}\right) J\left(p_{2}, p_{3}, p_{4}\right) \\
& \times J\left(p_{2}, p_{1}, p_{4}\right) J\left(p_{1}, p_{4}, p_{3}\right) \\
& \times J\left(p_{1}, p_{2} ; p_{3}, p_{4}\right) J\left(p_{2}, p_{3} ; p_{1}, p_{4}\right) J\left(p_{3}, p_{1} ; p_{2}, p_{4}\right) \\
& \times J\left(p_{3}, p_{4} ; p_{1}, p_{2}\right) J\left(p_{1}, p_{4} ; p_{2}, p_{3}\right) J\left(p_{2}, p_{4} ; p_{3}, p_{1}\right) .
\end{aligned}
$$

Proof. For part (1), let $\sigma$ be an odd permutation. Let $U$ be the union of two triangles along one of their edges. Let the first triangle have vertices $p_{1}, p_{2}, p_{3}$ and the second triangle have vertices $p_{3}, p_{2}, p_{1}$ with the opposite orientation. We can glue the two triangles along the edge $p_{1} p_{2}$. (Gluing along any other edge would lead to the same result for the corresponding generating series.) From the shuffle product formula (Theorem 3.21(iii)), it follows that $J(U)=J\left(p_{1}, p_{2}, p_{3}\right) J\left(p_{3}, p_{2}, p_{1}\right)$. (Note that the product is not the product in the ring $R$. It is induced by a shuffle product of iterated integrals on membranes.) From the second homotopy invariance theorem (Theorem 3.11) it follows that the generating series $J(U)$ depends on $U$ up to homotopy, which keeps the boundary components $p_{2} p_{3}, p_{3} p_{2}, p_{1} p_{3}$ and $p_{3} p_{1}$ on fixed unions of holomorphic curves. We can contract $U$ to its boundaries $\partial U$ so that the contracting homotopy keeps the boundary components on a fixed union of holomorphic curves. Therefore, $J(U)=J(\partial U)=1$.

Parts (2), (4) and (5) can be proven similarly.

For part (3), if $p_{1}, p_{2}, p_{3}, p_{4}$ belong to the same holomorphic (or antiholomorphic) curve, then the corresponding diangle has no interior, since the two edges will coincide. Recall that the edges of the diangle are defined via unique geodesic triangles lying on a holomorphic (or antiholomorphic) curve.

The proof of part (6) is essentially the same as the one for part (1); however, we will prove it independently, since it is a key property of the noncommutative Hilbert modular symbol. Consider a thickened tetrahedron with vertices $p_{1}, p_{2}, p_{3}, p_{4}$. 
The faces of the thickened tetrahedron are precisely the ones listed in the product of part (6). The whole product is equal to $J(V)$, where $V$ is the union of all faces of the thickened tetrahedron. From the second homotopy invariance theorem it follows that the generating series $J(V)$ depends on $V$ up to homotopy, which keeps the boundary components on a fixed union holomorphic curves. Since $V$ bounds a contractible 3-dimensional region (a thickened tetrahedron), from Theorem 3.11, it follows that $J(V)=J$ (point) $=1$.

4E. Definition of noncommutative Hilbert modular symbols. In this subsection, we define noncommutative Hilbert modular symbols. They are analogues of Manin's [2006] noncommutative modular symbol, applicable to the Hilbert modular group. Instead of the iterated path integrals that Manin uses, we use a higher-dimensional analogue, defined in Section 3.

Usually, a modular symbol represents a cohomology class. Manin's noncommutative modular symbol represents a noncommutative first cohomology class. We would like to say that the noncommutative Hilbert modular symbols represent noncommutative cohomology classes; this is formulated in Conjectures 4.14 and 4.15.

After defining the noncommutative Hilbert modular symbols, we prove some of their properties. These properties will be interpreted intuitively as cocycle or coboundary conditions. The approach in this subsection is more geometric. The purpose of presenting them here is to give many examples of relations and to help establish a suitable cohomology theory that truly captures these relations in a more structured way.

The cocycle interpretation is only for intuition; it is not precise. The formula holds for geometric reasons. Note that the composition is not the multiplication in the ring $R$; it is given by the shuffle product (see Theorem 3.21), which works for the generating series on iterated integrals on membranes. The multiplication is written linearly as we would multiply several elements in a group or in a ring; however, the multiplication is two-dimensional among regions with common boundaries.

In the next subsection will give some intuition about higher categories, for the purpose of giving more structure to the noncommutative Hilbert modular symbols and for a possible approach to defining a first and second noncommutative cohomology class.

For definitions of iterated integrals on membranes, see Definitions 3.4 and 3.5 for types $\boldsymbol{b}$ and $\boldsymbol{c}$ and Definition 4.9 for types $\boldsymbol{b}^{\prime}$ and $\boldsymbol{c}^{\prime}$.

Definition 4.13. We define noncommutative Hilbert modular symbols as generating series of iterated integrals on membranes of types $\boldsymbol{b}, \boldsymbol{c}, \boldsymbol{b}^{\prime}$, or $\boldsymbol{c}^{\prime}$ over a geodesic diangle by

$$
c_{p_{1}, p_{2} ; p_{3}}^{1}(\gamma)=J\left(p_{1}, p_{2} ; p_{3}, \gamma p_{3}\right)
$$


We also define noncommutative Hilbert modular symbols as generating series of iterated integrals on membranes of types $\boldsymbol{b}^{\prime}$ or $\boldsymbol{c}^{\prime}$ over a geodesic triangle by

$$
c_{p}^{2}(\gamma, \delta)=J(p, \gamma p, \gamma \delta p)
$$

where $p, p_{1}, p_{2}, p_{3}$ are cusp points in $\mathbb{H}^{2} \cup \mathbb{P}^{1}(K)$ and $\beta, \gamma, \delta \in \mathrm{SL}_{2}\left(\mathscr{O}_{K}\right)$.

We are going to define an action of $\mathrm{Mat}_{2}\left(\mathrm{O}_{K}\right)^{+}$on the generating series $J^{c}$, where $\operatorname{Mat}_{2}\left(\mathrm{O}_{K}\right)^{+}$is the semigroup of $2 \times 2$ matrices with totally positive determinant.

In order to interpret $c^{1}(\gamma)$ and $c^{2}(\gamma, \delta)$ as cocycles, we are going to define an action of the semigroup $\operatorname{Mat}_{2}\left(\mathrm{O}_{K}\right)^{+}$on the whole ring $R$ where the generating series take values. Such an action can be given via Hecke operators.

For simplicity, we shall assume that $\hat{O}_{K}$ has narrow class number 1 . We consider all Hecke eigenforms of weight $(2,2)$ with respect to $\mathrm{Mat}_{2}\left(\mathrm{O}_{K}\right)^{+}$. Now, let $u$ be a unit such that $u_{1}>0$ and $u_{2}<0$, where $u_{1}$ and $u_{2}$ are the images of $u$ under the two real embeddings of $K$ into $\mathbb{R}$. It exists, since the narrow class group is trivial. (For example, $K=\mathbb{Q}(\sqrt{2})$ is such a field.) We define an action of $\gamma \in \operatorname{Mat}_{2}\left(\mathscr{O}_{K}\right)$ on the ring $R$ (Definition 3.13) where the generating series takes values. We define

$$
\gamma \cdot f \mapsto T_{\gamma}(f)
$$

for $\gamma \in \operatorname{Mat}_{2}\left(\mathscr{O}_{K}\right)^{+}$. Let $f_{1}, \ldots, f_{m}$ be a basis of Hecke eigenforms of the space of cusp form of weight $(2,2)$. Let $X_{1}, Y_{1}, \ldots, X_{m}, Y_{m}$ be generators of $R$, and to each $f_{i}$ associate $X_{i}$ and $Y_{i}$. Then the action of $\gamma \in \operatorname{Mat}_{2}\left(\mathrm{O}_{K}\right)^{+}$is given by $T_{\gamma}\left(X_{i}\right)=$ $c\left(\gamma, f_{i}\right) X_{i}$ and $T_{\gamma}\left(Y_{i}\right)=Y_{i}$, where $c\left(\gamma, f_{i}\right)$ is the eigenvalue of the Hecke operator.

In this setting the group action, namely, the action of the Hilbert modular group, is trivial. This trivial action extend to the action of $T_{1}=$ id on the whole ring $R$. In fact, for an element $\beta \in \mathrm{SL}_{2}\left(O_{K}\right)$, the trivial action on $c_{p_{1}, p_{2} ; p_{3}}^{1}$ and $c_{p}^{2}$ can be realized as

$$
\left(\beta c_{p_{1}, p_{2} ; p_{3}}^{1}\right)(\gamma)=c_{\beta p_{1}, \beta p_{2} ; \beta p_{3}}^{1}(\beta \gamma)
$$

and

$$
\left(\beta c_{p}^{2}\right)(\gamma, \delta)=c_{\beta p}^{2}(\beta p, \beta \gamma p, \beta \gamma \delta p)
$$

The last two relations hold because for a cusp form of weight $(2,2)$ the differential form $f d z_{1} \wedge d z_{2}$ is invariant under the action of the Hilbert modular group $\Gamma$. Algebraically, for any geodesic diangle, we have

$$
\beta J\left(p_{1}, p_{2} ; p_{3}, p_{4}\right)=J\left(p_{1}, p_{2} ; p_{3}, p_{4}\right)=J\left(\beta p_{1}, \beta p_{2} ; \beta p_{3}, \beta p_{4}\right) .
$$

Similarly, for a geodesic triangle,

$$
\beta J\left(p_{1}, p_{2}, p_{3}\right)=J\left(p_{1}, p_{2}, p_{3}\right)=J\left(\beta p_{1}, \beta p_{2}, \beta p_{3}\right) .
$$


The relations among the symbols are based on two properties: composition via shuffle product Theorem 3.21(iii) and homotopy invariance (Theorems 3.7 and 3.11).

Conjecture 4.14. The noncommutative Hilbert modular symbol $c_{p_{1}, p_{2} ; p_{3}}^{1}$ is a 1cocycle. Moreover, if we change the point $p_{3}$ to $q_{3}$, then the cocycle changes by a coboundary.

Property (5) of Theorem 4.12 can be interpreted as a 1-cocycle relation. Consider the analogy with a noncommutative 1-cocycle of a group acting on a noncommutative ring; we define the boundary of $c_{p_{1}, p_{2} ; p_{3}}^{1}$ by

$$
d c_{p_{1}, p_{2} ; p_{3}}^{1}(\beta, \gamma)=c_{p_{1}, p_{2} ; p_{3}}^{1}(\beta)\left(\beta c_{p_{1}, p_{2} ; p_{3}}^{1}\right)(\gamma)\left(c_{p_{1}, p_{2} ; p_{3}}^{1}(\beta \gamma)\right)^{-1} \text {. }
$$

The action of $\beta$ on the cocycle is given in Definition 4.13. In contrast to a first noncommutative cocycle (see for example [Brown 1982]), here we have twodimensional composition of symbols, that is, one can compose the symbols as two-morphisms in a two-category.

Then

$$
\begin{aligned}
d c_{p_{1}, p_{2} ; p_{3}}^{1}(\beta, \gamma) & =J\left(p_{1}, p_{2} ; p_{3}, \beta p_{3}\right)\left(\beta J\left(p_{1}, p_{2} ; p_{3}, \gamma p_{3}\right)\right) J^{-1}\left(p_{1}, p_{2} ; p_{3}, \beta \gamma p_{3}\right) \\
& =J\left(p_{1}, p_{2} ; p_{3}, \beta p_{3}\right) J\left(p_{1}, p_{2} ; \beta p_{3}, \beta \gamma p_{3}\right) J^{-1}\left(p_{1}, p_{2} ; p_{3}, \beta \gamma p_{3}\right) \\
& =1 .
\end{aligned}
$$

If we change $p_{3}$ to $q_{3}$ then the cocycle changes by a coboundary. Let $b^{0}=$ $J\left(p_{1}, p_{2} ; p_{3}, q_{3}\right)$ be a 0 -cochain. Then

$$
\begin{aligned}
c_{p_{1}, p_{2} ; q_{3}}^{1}(\gamma) & =J\left(p_{1}, p_{2} ; p_{3}, \gamma p_{3}\right) \\
& =J\left(p_{1}, p_{2} ; p_{3}, q_{3}\right) J\left(p_{1}, p_{2} ; q_{3}, \gamma q_{3}\right) J\left(p_{1}, p_{2} ; \gamma q_{3}, \gamma p_{3}\right) \\
& =J\left(p_{1}, p_{2} ; p_{3}, q_{3}\right) J\left(p_{1}, p_{2} ; q_{3}, \gamma q_{3}\right)\left(\gamma J\left(p_{1}, p_{2} ; p_{3}, q_{3}\right)\right)^{-1} \\
& =b^{0} c_{p_{1}, p_{2} ; q_{3}}^{1}(\gamma)\left(\gamma b^{0}\right)^{-1}
\end{aligned}
$$

Conjecture 4.15. The noncommutative Hilbert modular symbol $c_{p}^{2}(\beta, \gamma)$ satisfies a 2-cocycle relation. Moreover, if we change the point $p$ to $q$, then the cocycle changes by a coboundary up to terms involving $c^{1}$.

Recall

$$
c_{p}^{2}(\beta, \gamma)=J(p, \beta p, \beta \gamma p)
$$


Then $c_{p}^{2}$ satisfies a 2-cocycle condition up to a multiple of the 1-cocycle $c_{q_{1}, q_{2} ; q_{3}}^{1}$ for various points $q_{1}, q_{2}, q_{3}$. For the 2-cocycle relation, we compute $d c_{p}^{2}(\beta, \gamma, \delta)$ :

$$
\begin{aligned}
d c_{p}^{2}(\beta, \gamma, \delta)= & c_{p}^{2}(\beta, \gamma) c^{2}(\beta, \gamma \delta)\left(c^{2}(\beta \gamma, \delta)\right)^{-1}\left(\beta \cdot c^{2}(\gamma, \delta)\right)^{-1} \\
= & J(p, \beta p, \beta \gamma p) J(p, \beta p, \beta \gamma \delta p) \\
& \times J(p, \beta \gamma p, \beta \gamma \delta p)^{-1} J(\beta p, \beta \gamma p, \beta \gamma \delta p)^{-1} .
\end{aligned}
$$

In order to have $d c_{p}^{2}(\beta, \gamma, \delta)=1$, we must multiply by suitable values of $c^{1}$, corresponding to edges of a certain thickened tetrahedron. Then

$$
\begin{gathered}
d c_{p}^{2}(\beta, \gamma, \delta) \times\left[c_{p, \beta p ; \beta \gamma p}^{1}\left((\beta \gamma) \delta(\beta \gamma)^{-1}\right) c_{\beta p, \beta \gamma p ; p}^{1}(\beta \gamma \delta) c_{\beta \gamma p, p ; \beta p}^{1}\left((\beta) \gamma \delta \beta^{-1}\right)\right. \\
\left.\times c_{\beta \gamma p, \beta \gamma \delta p ; p}^{1}(\beta) c_{p, \beta \gamma \delta p ; \beta p}^{1}\left(\beta \gamma \beta^{-1}\right) c_{\beta p, \beta \gamma \delta p ; \beta \gamma p}^{1}\left((\beta \gamma)^{-1}\right)\right] \\
=\left[c_{p}^{2}(\beta, \gamma) c^{2}(\beta, \gamma \delta)\left(c^{2}(\beta \gamma, \delta)\right)^{-1}\left(\beta \cdot c^{2}(\gamma, \delta)\right)^{-1}\right] \\
\times\left[c_{p, \beta p ; \beta \gamma p}^{1}\left((\beta \gamma) \delta(\beta \gamma)^{-1}\right) c_{\beta p, \beta \gamma p ; p}^{1}(\beta \gamma \delta) c_{\beta \gamma p, p ; \beta p}^{1}\left((\beta) \gamma \delta \beta^{-1}\right)\right. \\
\left.\quad \times c_{\beta \gamma p, \beta \gamma \delta p ; p}^{1}(\beta) c_{p, \beta \gamma \delta p ; \beta p}^{1}\left(\beta \gamma \beta^{-1}\right) c_{\beta p, \beta \gamma \delta p ; \beta \gamma p}^{1}\left((\beta \gamma)^{-1}\right)\right] \\
=\left[\begin{array}{l}
\left.J(p, \beta p, \beta \gamma p) J(p, \beta p, \beta \gamma \delta p) J(p, \beta \gamma p, \beta \gamma \delta p)^{-1} J(\beta p, \beta \gamma p, \beta \gamma \delta p)^{-1}\right] \\
\times[
\end{array}\right] \\
\quad \times J(p, \beta p ; \beta \gamma p, \beta \gamma \delta p) J(\beta p, \beta \gamma p ; p, \beta \gamma \delta p) J(\beta \gamma p, p ; \beta p, \beta \gamma \delta p) \\
=1 .
\end{gathered}
$$

The first equality follows from (28). The second equality follows from the definition of the symbols. And the last equality follows from property (6) of Theorem 4.12 with $\left(p_{1}, p_{2}, p_{3}, p_{4}\right)=(p, \beta p, \beta \gamma p, \beta, \gamma \delta p)$. Therefore, we obtain that $d c_{p}^{2}(\beta, \gamma, \delta)$ is 1 up to values of the 1 -cocycle $c^{1}$.

Conjecture 4.16. The conjectural cocycles $c_{p}^{2}$ and $c_{q}^{2}$ are homologous:

$$
c_{p}^{2}(\beta, \gamma)=c_{q}^{2}(\beta, \gamma)\left[d b_{p q}^{1}(\beta, \gamma)\right] \prod_{i} J\left(D_{i}\right),
$$

up to a product of $J\left(D_{i}\right)$, where the $D_{i}$ are geodesic diangles.

Before we proceed, we would like to make an analogy between 1-dimensional and 2-dimensional cocycles. For the 1-dimensional cocycle, the property that it is a cocycle uses the geometry of a triangle, where the faces of the triangle are essentially the 1-cocycle. We want commutativity of the triangular diagram. We think of the commutativity of the diagram as follows: consider the interior of the triangle as a homotopy of paths and think of the 1-cocycle as a homotopy-invariant function. The 2-cocycle relation is represented by the faces of a tetrahedron. By "commutativity" of the diagram, we mean a homotopy invariant 2-cocycle and a homotopy from one of the faces to the union of the other three faces. 
The comparison that $c_{p_{1}, p_{2} ; p_{3}}^{1}$ and $c_{p_{1}, p_{2} ; q_{3}}^{1}$ are homologous is given by a squareshaped diagram. The analogy with dimension 2 is that the cocycles $c_{p}^{2}$ and $c_{q}^{2}$ are two faces of an octahedron. The vertices associated to $c_{p}^{2}(\beta, \gamma)$ are $(p, \beta p, \beta \gamma p)$ and the vertices associated to $c_{q}^{2}$ are $(q, \beta q, \beta \gamma q)$. The two faces will be opposite to each other on the octahedron Oct so that the three pairs of opposite vertices are $(p, \beta \gamma q),(\beta p, q)$ and $(\beta \gamma p, \beta q)$. The remaining six faces are combined into two triples. Each of them corresponds to a coboundary of a 1-chain.

Let

$$
b_{p, q}^{1}(\beta)=[J(p, q, \beta p) J(q, \beta q, \beta p)][J(q, \beta p ; p, \beta q)] .
$$

Consider the action of $\gamma \in \Gamma$ on $b^{1}$ by acting on each point in the argument of $J$, denoted as before by $\gamma \cdot b^{1}$. Then, we define

$$
d b_{p, q}^{1}(\beta, \gamma)=b_{p, q}^{1}(\beta)\left[\beta \cdot b_{p, q}^{1}(\gamma)\right]\left[b_{p, q}^{1}(\beta \gamma)\right]^{-1},
$$

where $\beta \cdot b_{p, q}^{1}(\gamma)=[J(\beta p, \beta q, \beta \gamma p) J(\beta q, \beta \gamma q, \beta \gamma p)][J(\beta q, \beta \gamma p ; p ; \beta \gamma q)]$.

Consider the above octahedron Oct. Remove from it the tetrahedron $T$ with vertices $(p, q, \beta \gamma q, \beta \gamma p)$. Then the triangles of the remaining geometric figure are precisely the triangles in the definitions of $c_{p}^{2}(\beta, \gamma), c_{q}^{2}(\beta, \gamma)$ and $d b_{p, q}^{1}(\beta, \gamma)$. Now, consider thickenings of the edges, which are common for two triangles. It can be done in the following way. Instead of any triangle, we can take a geodesic triangle. The two triangles that had a common edge might have only two common vertices. Then the region between the two geodesic, one for each of the geodesic triangles, forms the induced diangle. Take $J$ of the induces diangles from the octahedron Oct and $J^{-1}$ of the induced diangles from the tetrahedron $T$. Their product gives $\prod_{i} J\left(D_{i}\right)$. The equality holds because we apply $J$ to the union of the faces of the thickened Oct $-T$, which gives 1 .

4F. A two-category. Why do we need a two-category? Is there an example of a sheaf on this category/topology? How does the noncommutative Hilbert modular symbols represents a sheaf?

The ideas presented in this subsection will be developed in a follow-up paper. Here we present the basic constructions that give justification for the conjectures that the noncommutative Hilbert modular symbols $c^{1}$ and $c^{2}$ are cocycles in some categorical and sheaf-theoretic setting. For sheaves on 2-categories one may consult [Street 1982]. Since our 2-morphisms are invertible one may also use Lurie's constructions [2009] of sheaves on higher categories.

We are going to construct a 2-category $C$ and a sheaf $J$ on $C$. We define $p$ to be an object of the 2-category $C$ if $p$ is a cusp point, that is $p \in \mathbb{P}^{1}(K)$. We define 1 -morphisms in the following way. Let $\sigma$ be the geodesic connecting 0 and $\infty$ that lies on the diagonal $\Delta=i(\mathbb{M}) \subset \mathbb{H} \times \mathbb{H}$. There is unique such geodesic. All geodesics $\gamma^{*} \sigma$ together with a choice of orientation are defined to be 1-morphisms, 
where $\gamma \in \mathrm{PGL}_{2}(K)$. We define the 1-morphisms of $C$ to be finite concatenations of geodesics of type $\gamma^{*} \sigma$ or the trivial path whose image coincides with a cusp point. Consider ideal triangles and ideal diangles as cells from which we build manifolds with corners. A 2-morphism is a finite union of manifolds with corners, made from finitely many ideal diangles and ideal triangles, which is path-connected and has orientation.

The boundary of a 1-morphism is a union of two objects - the starting point and the ending point of the directed path. The boundary of a 2-morphism (a 2manifold with corners) is a finite union of 1-morphisms (oriented loops), where the orientation of the loops on the boundary is induced by the orientation of the 2-manifold with corners.

Now we are going to define a 2-sheaf $J$, whose values on a 2-morphism will be in a subset of the ring $R$ and whose values on an object and on a 1-morphism will be a subset of a countable product of the ring $R$ with itself.

As always, $S_{2,2}(\Gamma)$ denotes the space of cusp forms of weight $(2,2)$ with respect to the group $\Gamma$. Here we will consider this space as the space of holomorphic 2-forms on $\mathbb{H} \times \mathbb{W}$ which vanish on the cusps and which can descend to the Hilbert modular surface $X_{\Gamma}=\Gamma \backslash\left(\mathbb{T}^{2} \cup \mathbb{P}^{1}(K)\right)$. Every $n$-tuple of such holomorphic forms $\Omega \in\left(S_{2,2}(\Gamma)\right)^{n}$ defines a value of a 2-morphism $f$ in $C$. Let this value be the generating series $J_{f}(\Omega)$. Let $J_{f}$ be the collection of all values $J_{f}(\Omega)$ for $\Omega \in\left(S_{2,2}(\Gamma)\right)^{n}$. Let $e$ be a 1-morphism. We say that $e$ is in the boundary of a 2-morphism $f$, denoted $e \subset \partial f$, if the image of the loop $e$ is in the boundary of the image of the membrane $f$ together with the induced orientation on $e$ from $f$. We say that an object $p$ is in the boundary of a 1-morphism $e$, denoted by $p \in \partial e$, if $p$ is a source or a target of $e$. We define the values of $J$ on a 1-morphism $e$ to be the product

$$
\prod_{e \subset \partial f} J_{f} \subset \prod_{e \subset \partial f} R
$$

We define the values of $J$ on objects $p$ to be

$$
\prod_{\substack{p \in \partial e \\ e \subset \partial f}} J_{f} \subset \prod_{\substack{p \in \partial e \\ e \subset \partial f}} R .
$$

The sheaf conditions for 1- and 2-morphisms resemble the classical conditions for a presheaf to be a sheaf.

Let $f_{i}: A_{i} \rightarrow[0,1]^{2}$ be a finite collection of disjoint 2-morphisms, whose union is a morphism $f: A \rightarrow[0,1]^{2}$. We define a finite collection $f_{i j}^{k}$ of 1-morphisms and 0-morphisms (objects) such that the union $\bigcup_{k} \operatorname{im}\left(f_{i j}^{k}\right)=\operatorname{im}\left(f_{i}\right) \cap \operatorname{im}\left(f_{j}\right)$ is a disjoint union of the intersection. 
Then the equalizer

$$
J_{f} \rightarrow \prod_{i} J_{f_{i}} \rightrightarrows \prod_{i j k} J_{f_{i j}^{k}}
$$

is exact (for a definition of equalizer one may consult [Borceux 1994]).

Similarly, let $e$ be a 1-morphism and let $\left\{e_{i}\right\}$ be a finite set of disjoint 1morphisms such that the union $\bigcup_{i} \operatorname{im}\left(e_{i}\right)$ is equal to $\operatorname{im}(e)$. We can write the intersection $\operatorname{im}\left(e_{i}\right) \cap \operatorname{im}\left(e_{j}\right)$ as a finite union of 0-morphisms $\bigcup_{k} \operatorname{im}\left(e_{i j}^{k}\right)$, for some 1-morphisms $e_{i j}^{k}$.

Then the equalizer

$$
J_{e} \rightarrow \prod_{i} J_{e_{i}} \rightrightarrows \prod_{i j k} J_{e_{i j}^{k}}
$$

is exact.

The cochain is defined as

$$
\prod_{p: 0 \text {-morph }} J_{p} \rightarrow \prod_{e: 1 \text {-morph }} J_{e} \rightarrow \prod_{f: 2 \text {-morph }} J_{f} \rightarrow \prod_{\substack{g: 2 \text {-morph } \\ \partial g=\varnothing}} J_{g}
$$

The maps $J_{e} \rightarrow J_{p}$ and $J_{p} \rightarrow J_{f}$ are surjective when they are defined, resembling flabby sheaves. Thus, we should have trivial zeroth and first cohomology set. The only nontrivial cohomology will be the second cohomology set. The cocycle conditions for both noncommutative Hilbert modular symbols $c^{1}$ and $c^{2}$ can be interpreted as a particular case of maps

$$
\prod_{f: 2 \text {-morph }} J_{f} \rightarrow \prod_{\substack{g: 2 \text {-morph } \\ \partial g=\varnothing}} J_{g}
$$

For $c^{1}$, the boundary condition is that a union of two diangles with a common edge is a third diangle. One can think of the these three diangles as the boundary of a degenerate 3-dimensional region. One can realize this cocycle condition as a sheaf-theoretic one by modifying the above definition so that the 2-morphisms consists of a finite union of ideal diangles (without using the ideal triangles). Then the sheaf-theoretic second cocycle condition is the one for noncommutative Hilbert modular symbol $c^{1}$.

If we are able to quotient the 2-category described in the beginning of this subsection by the 2-morphisms generated by diangles, then we have only two morphisms generated by ideal triangles. The noncommutative Hilbert modular symbol $c^{2}$ is exactly the one that considers ideal triangles. Note that its cocycle relation for $c^{2}$ is satisfied up to 2-morphisms generated by diangles. 
4G. Explicit computations. Multiple Dedekind zeta values. In this subsection, we make explicit computations of some ingredients in the noncommutative Hilbert modular symbol. Manin [2006] compared explicit formulas of integrals in the noncommutative modular symbol to multiple zeta values. The similarities are in terms of both infinite series formulas and formulas via iterated path integrals. Here we compare certain integrals in the noncommutative Hilbert modular symbol to multiple Dedekind zeta values (for multiple Dedekind zeta values, see [Horozov 2014b]). Again the similarities are in terms of both infinite series formulas and formulas via iterated integrals over membranes.

We are going to consider the Fourier expansion of two Hilbert cusp forms $f$ and $g$. Let $\omega_{f}=f d z_{1} \wedge d z_{2}, \omega_{g}=g d z_{1} \wedge d z_{2}$ and $\omega_{0}=d z_{1} \wedge d z_{2}$. We are going to associate $L$-values to iterated integrals of the forms $\omega_{f}$ and $\omega_{g}$. The $L$-values will be iterated integrals over an union of diangles. One can think of a diangle connecting 0 and $\infty$ as a segment or as a real cone. The union will be a disjoint union of all such real cones connecting 0 and $\infty$ or simply $\operatorname{Im}(\mathbb{M}) \times \operatorname{Im}(\mathbb{M})$. We also recall the definition of a multiple Dedekind zeta values via (discrete) cones. Finally, we prove analogous formulas for iterated $L$-values associated to Hilbert cusp forms and for multiple Dedekind zeta values.

We will be mostly interested in the modular symbol associated to a diangle. Let us recall what we mean by a diangle.

Let $p_{1}, p_{2}, p_{3}, p_{4}$ be four cusp points. Let $\gamma_{1} \in \mathrm{GL}_{2}(K)$ be a linear fractional transformation that sends $\gamma_{1}\left(p_{1}\right)$ to $0, \gamma_{1}\left(p_{2}\right)$ to $\infty$, and $\gamma_{1}\left(p_{3}\right)$ to 1 . Let $\Delta$ be the image of the diagonal embedding of $\mathbb{M}^{1}$ into $\mathbb{U}^{2}$. Then 0,1 and $\infty$ are boundary points of $\Delta$. Let $\lambda(0, \infty)$ be the unique geodesic in $\Delta$ that connects 0 and $\infty$. And let

$$
\lambda_{1}\left(p_{1}, p_{2}\right)=\gamma_{1}^{-1} \lambda(0, \infty)
$$

be the pullback of the geodesic $\lambda$ to a geodesic connecting $p_{1}$ and $p_{2}$.

Now consider the triple $p_{1}, p_{2}$ and $p_{4}$. Let $\gamma_{2} \in \mathrm{GL}_{2}(K)$ be a linear fractional transformation that sends $\gamma_{2}\left(p_{1}\right)$ to $0, \gamma_{2}\left(p_{2}\right)$ to $\infty$ and $\gamma_{2}\left(p_{4}\right)$ to 1 . Let $\Delta$ be the image of the diagonal embedding of $\mathbb{H}^{1}$ into $\mathbb{H}^{2}$. Then 0,1 and $\infty$ are boundary points of $\Delta$. Let $\lambda(0, \infty)$ be the unique geodesic in $\Delta$ that connects 0 and $\infty$. And let

$$
\lambda_{2}\left(p_{1}, p_{2}\right)=\gamma_{2}^{-1} \lambda(0, \infty)
$$

be the pullback of the geodesic $\lambda$ to a geodesic connecting $p_{1}$ and $p_{2}$.

By a diangle, we mean a region in $\mathbb{T}^{2} \cup \mathbb{P}^{1}(K)$ with the homotopy type of a disc, bounded by the geodesics $\lambda_{1}(0, \infty)$ and $\lambda_{2}(0, \infty)$.

We are going to present a computation for the diangle $D_{u}$ defined by the points $\left(0, \infty, u^{1}, u^{-1}\right)$, where $u$ is a generator for the group of units modulo \pm 1 in $K$. Let (1) be the trivial permutation. 
Lemma 4.17. Let $u$ be a totally positive unit. Then

$$
\iint_{D_{u}}^{(1)(1)} e^{2 \pi i\left(\alpha_{1} z_{1}+\alpha_{2} z_{2}\right)} d z_{1} \wedge d z_{2}=\frac{1}{(2 \pi i)^{2}} \frac{u_{2}^{2}-u_{1}^{2}}{\left(\alpha_{1} u_{1}+\alpha_{2} u_{2}\right)\left(\alpha_{1} u_{2}+\alpha_{2} u_{1}\right)} .
$$

Proof. Let $u_{1}$ and $u_{2}$ be the two embeddings of $u$ into $\mathbb{R}$. Then $(0, \infty, u)$ can be mapped to $(0, \infty, 1)$ by $\gamma_{1}=\left(\begin{array}{cc}u^{-1} & 0 \\ 0 & 1\end{array}\right)$. The geodesic $\lambda(0, \infty)$ can be parametrized by $(i t, i t)$ for $t \in \mathbb{R}$. Then the geodesic $\lambda_{1}(0, \infty)$ on the geodesic triangle $(0, \infty, u)$ can be parametrized by $\left\{\left(i u_{1} t, i u_{2} t\right) \mid t>0\right\}$. Similarly, the geodesic $\lambda_{2}(0, \infty)$ on the geodesic triangle $\left(0, \infty, u^{-1}\right)$ can be parametrized by $\left\{\left(i u_{2} t, i u_{1} t\right) \mid t>0\right\}$. Then the diangle $D_{u}$ can be parametrized by

$$
\left\{\left(z_{1}, z_{2}\right) \in \mathbb{M}^{2} \mid \operatorname{Re}\left(z_{1}\right)=\operatorname{Re}\left(z_{2}\right)=0, \operatorname{Im}\left(z_{1}\right) \in\left(\frac{u_{1}}{u_{2}} t, \frac{u_{2}}{u_{1}} t\right), \operatorname{Im}\left(z_{2}\right)=t \in(0, \infty)\right\} .
$$

Then we have

$$
\begin{aligned}
\iint_{D_{u}}^{(1)(1)} e^{2 \pi i\left(\alpha_{1} z_{1}+\alpha_{2} z_{2}\right)} d z_{1} \wedge d z_{2} & =\int_{\infty}^{0}\left(\int_{\frac{u_{2}}{u_{1}} t}^{\frac{u_{1}}{u_{2}} t} e^{2 \pi i\left(\alpha_{1} z_{1}+\alpha_{2} t\right)} d z_{1}\right) d t \\
& =\frac{1}{2 \pi i \alpha_{1}} \int_{\infty}^{0}\left(e^{\alpha_{1} \frac{u_{1}}{u_{2}} t+\alpha_{2} t}-e^{\alpha_{1} \frac{u_{2}}{u_{1}} t+\alpha_{2} t}\right) d t \\
& =\frac{1}{(2 \pi i)^{2}} \frac{1}{\alpha_{1}}\left(\frac{1}{\alpha_{1} \frac{u_{1}}{u_{2}}+\alpha_{2}}-\frac{1}{\alpha_{1} \frac{u_{2}}{u_{1}}+\alpha_{2}}\right) \\
& =\frac{1}{(2 \pi i)^{2}} \frac{u_{2}^{2}-u_{1}^{2}}{\left(\alpha_{1} u_{1}+\alpha_{2} u_{2}\right)\left(\alpha_{1} u_{2}+\alpha_{2} u_{1}\right)}
\end{aligned}
$$

Therefore, one term of the Fourier expansion of a Hilbert cusp form paired with a symbol given by one diangle does not resemble a norm of an algebraic integer. However, if we integrate over an infinite union of diangles, then a similarity with Dedekind zeta and multiple Dedekind zeta values occurs.

Consider the limit of $D_{u^{n}}$ when $n \rightarrow \infty$. It is the product of the two imaginary axes of the two upper half-planes. Set

$$
\operatorname{Im}\left(\mathbb{M}^{2}\right)=\operatorname{Im}(\mathbb{M}) \times \operatorname{Im}(\mathbb{M}) .
$$

One can think of this region as an infinite union of diangles.

Denote by $\alpha z$ the sum of products $\alpha_{1} z_{1}+\alpha_{2} z_{2}$. Using the methods of [Horozov 2014b, Section 1], we obtain

$$
\frac{(2 \pi i)^{-2}}{N(\alpha) N(\alpha+\beta)}=\int_{\operatorname{Im}\left(\mathbb{H}^{2}\right)}^{(1)(1)} e^{2 \pi i \alpha z} d z_{1} \wedge d z_{2} \cdot e^{2 \pi i \beta z} d z_{1} \wedge d z_{2}
$$


and

$$
\begin{aligned}
& \frac{1}{(2 \pi i)^{2}} \frac{1}{N(\alpha)^{3} N(\alpha+\beta)^{2}} \\
& \quad=\int_{\operatorname{Im}\left(\mathbb{M} \mathbb{R}^{2}\right)}^{(1)(1)} e^{2 \pi i \alpha z} d z_{1} \wedge d z_{2} \cdot\left(d z_{1} \wedge d z_{2}\right) \cdot\left(d z_{1} \wedge d z_{2}\right) \cdot e^{2 \pi i \beta z} d z_{1} \wedge d z_{2} \cdot\left(d z_{1} \wedge d z_{2}\right) .
\end{aligned}
$$

Let $f$ and $g$ be two cusp forms of weights $(2 k, 2 k)$ and $(2 l, 2 l)$, respectively. Consider the Fourier expansion of both of the cusp forms. Let

$$
f=\sum_{\alpha \gg 0} a_{\alpha} e^{2 \pi i \alpha z} \quad \text { and } \quad g=\sum_{\beta \gg 0} b_{\beta} e^{2 \pi i \beta z} .
$$

Since $f$ is of weight $(2 k, 2 k)$, we have that $a_{u \alpha}=a_{\alpha}$, where $u$ is a unit. For such a modular form the modular factor with respect to the transformation $z \rightarrow u z$ is 1 . The $L$-values of $f$ are

$$
\begin{aligned}
L_{f}(n) & =\int_{\operatorname{Im}\left(\mathbb{H}^{2}\right)}^{(1)(1)} \sum_{\alpha \in \mathbb{O}_{K}^{+} / U^{+}} a_{\alpha} e^{2 \pi i \alpha z} d z_{1} \wedge d z_{2} \cdot\left(d z_{1} \wedge d z_{2}\right)^{\cdot(n-1)} \\
& =\frac{1}{(2 \pi i)^{2 n}} \sum_{\alpha \in \mathbb{O}_{K}^{+} / U^{+}} \frac{a_{\alpha}}{N(\alpha)^{n}} .
\end{aligned}
$$

Here $\mathrm{O}_{K}^{+}$denotes the totally positive algebraic integers in $K$ and $U^{+}$denotes the totally positive units.

We recall some of the definitions from [Horozov 2014b]. We fix a positive cone $C$ in $\mathrm{O}_{K}$, by which we mean

$$
C=\mathbb{N} \cup\left\{\alpha \in \mathbb{O}_{K} \mid a+b \epsilon, a, b \in \mathbb{N}\right\},
$$

where $\epsilon$ is a generator of the group of totally positive units. By $\epsilon^{k} C$, we mean the collection of products $\epsilon^{k} \alpha$, where $\alpha$ varies in the cone $C$.

The following infinite sum is an example of a multiple Dedekind zeta value:

$$
\zeta_{K ; C, \epsilon^{k} C}(m, n)=\sum_{\alpha \in C} \sum_{\beta \in \epsilon^{k} C} \frac{1}{N(\alpha)^{m} N(\alpha+\beta)^{n}} .
$$

Let $Z(m, n)=\sum_{k \in \mathbb{Z}} \zeta_{K ; C, \epsilon^{k} C}(m, n)$, where $C$ is any set representing the totally positive algebraic integers $\mathrm{O}_{K}^{+}$modulo totally positive units $U^{+}$.

Lemma 4.18. The values $Z(m, n)$ are finite for $m>n>1$. 
Proof. Let $\epsilon$ be a generators of the group of totally positive units $U^{+}$in $K$. For the two real embeddings $\epsilon_{1}$ and $\epsilon_{2}$ of $\epsilon$, we can assume that $\epsilon_{1}>1>\epsilon_{2}$. Otherwise we can take its reciprocal.

$$
\begin{aligned}
Z(m, n) & =\sum_{k \in Z} \sum_{\alpha, \beta \in C} \frac{1}{N(\alpha)^{m} N\left(\alpha+\epsilon^{k} \beta\right)^{n}} \\
& <\sum_{\alpha, \beta \in C} \frac{1}{N(\alpha)^{m}}\left(\frac{1}{N(\alpha+\beta)^{n}}+\sum_{k=1}^{\infty} \frac{2^{n}}{\epsilon_{1}^{k}}\left(\frac{1}{\alpha_{1}^{n} \beta_{2}^{n}}+\frac{1}{\alpha_{2}^{n} \beta_{1}^{n}}\right)\right) \\
& <\sum_{\alpha, \beta \in C} \frac{1}{N(\alpha)^{m}}\left(\frac{1}{N(\alpha+\beta)^{n}}+\sum_{k=1}^{\infty} \frac{2}{\epsilon_{1}^{k}}\left(\frac{N(\alpha+\beta)^{n}-N(\alpha)^{n}}{N(\alpha+\beta)^{n}}\right)\right) \\
& =\sum_{\alpha, \beta \in C} \frac{1}{N(\alpha)^{m}}\left(\frac{1}{N(\alpha+\beta)^{n}}+\frac{2}{\epsilon_{1}-1}\left(\frac{N(\alpha+\beta)^{n}-N(\alpha)^{n}}{N(\alpha+\beta)^{n}}\right)\right) \\
& =\sum_{\alpha, \beta \in C} \frac{1}{N(\alpha)^{m} N(\alpha+\beta)^{n}}+\frac{2}{\epsilon_{1}-1} \frac{1}{N(\alpha)^{n}}-\frac{2}{N(\alpha)^{m-n} N(\alpha+\beta)^{n}} \\
& =\zeta_{K}(C ; m, n)-\frac{2}{\epsilon_{1}-1}\left(\zeta_{K}(C ; n)+\zeta_{K}(C ; m-n, n)\right) .
\end{aligned}
$$

Equation (29) is the definition. Inequality (30) is based on the following: $\epsilon_{2}<1$ is replaced with 1 when $k>0$. For $k<0$ we use $\epsilon_{2}^{k}=\epsilon_{1}^{-k}$. We put 1 for $\epsilon_{1}^{k}$ for $k<0$. The case $k=0$ is treated separately. Finally we group the terms with equal powers of $\epsilon_{1}$. In inequality (31) we estimate the mixed terms in the brackets. In (32) we take the sum of the geometric series in $\epsilon_{1}^{-1}$. Then in (33) we open the brackets. And finally, in (34), we express the sums as a finite linear combinations of a Dedekind zeta value and multiple Dedekind zeta values.

The following definition of an iterated $L$-value is a coefficient of one monomial from the noncommutative Hilbert modular symbol of type $\boldsymbol{b}$ :

Definition 4.19. For a pair of Hilbert cusp forms $f$ and $g$ with Fourier expansions

$$
f=\sum_{\alpha \gg 0} a_{\alpha} e^{2 \pi i \alpha z} \quad \text { and } \quad g=\sum_{\beta \gg 0} b_{\beta} e^{2 \pi i \beta z}
$$

we define iterated $L$-values

$$
\begin{array}{r}
L_{f, g}(m, n)=\int_{\operatorname{Im}\left(\mathbb{H}^{2}\right)}^{(1)(1)} \sum_{(\alpha, \beta) \in\left(\mathbb{O}_{K}^{+}, O_{K}^{+}\right) / U}\left(a_{\alpha} e^{2 \pi i \alpha z} d z_{1} \wedge d z_{2}\right) \cdot\left(d z_{1} \wedge d z_{2}\right)^{\cdot(m-1)} \\
\cdot\left(b_{\beta} e^{2 \pi i \beta z} d z_{1} \wedge d z_{2}\right) \cdot\left(d z_{1} \wedge d z_{2}\right)^{\cdot(n-1)} .
\end{array}
$$


Theorem 4.20. Using the above definition, we have

$$
L_{f, g}(m, n)=\sum_{k \in \mathbb{Z}} \sum_{\alpha \in C, \beta \in \epsilon^{k} C} \frac{a_{\alpha} b_{\beta}}{N(\alpha)^{m} N(\alpha+\beta)^{n}} .
$$

Proof.

$$
\begin{aligned}
L_{f, g}(m, n) & =\int_{\operatorname{Im}\left(\mathbb{H}^{2}\right)}^{(1)(1)} \sum_{(\alpha, \beta) \in\left(\mathbb{O}_{K}^{+}, \mathbb{O}_{K}^{+}\right) / U}\left(a_{\alpha} e^{2 \pi i \alpha z} d z_{1} \wedge d z_{2}\right) \cdot\left(d z_{1} \wedge d z_{2}\right)^{\cdot(m-1)} \\
& \left.=\sum_{(\alpha, \beta) \in\left(\mathbb{O}_{K}^{+}, \mathbb{O}_{K}^{+}\right) / U} \frac{a_{\alpha} b_{\beta}}{N(\alpha)^{m} N(\alpha+\beta)^{n}} d z_{1} \wedge d z_{2}\right) \cdot\left(d z_{1} \wedge d z_{2}\right)^{\cdot(n-1)} \\
& =\sum_{k \in \mathbb{Z} ; \alpha, \beta \in C} \frac{a_{\alpha} b_{\beta}}{N(\alpha)^{m} N\left(\alpha+\epsilon^{k} \beta\right)^{n}} \\
& =\sum_{k \in \mathbb{Z}, \alpha \in C} \sum_{\beta \in \epsilon^{k} C} \frac{a_{\alpha} b_{\beta}}{N(\alpha)^{m} N(\alpha+\beta)^{n}}
\end{aligned}
$$

We would like to bring to the attention of the reader Definition 4.19, the definition of the multiple $L$-values. More specifically, we would like to point out that the region of integration is an infinite union of diangles (or equivalently an infinite union of real cones; see the beginning of this section). Note also that in Theorem 4.20 the values of the multiple $L$-functions are expressed as an infinite sums over different discrete cones, namely, over $\epsilon^{k} C$ for $k \in \mathbb{Z}$. However, a single real cone $D_{u}$, as in Lemma 4.18, does not correspond to a single discrete cone. Only a good union of real cones $\operatorname{Im}(\mathbb{M}) \times \operatorname{Im}(\mathbb{H})$ corresponds to a good union of discrete cones $\bigcup_{k \in \mathbb{Z}}\left(C, \epsilon^{k} C\right)$ as a fundamental domain of $\left(\mathcal{O}_{K}^{+}, \mathcal{O}_{K}^{+}\right) / U^{+}$.

\section{Acknowledgements}

I would like to thank Ronnie Brown for his talk on higher categories at the University of Durham, which helped me to define iterated integrals over membranes. I would like to thank Mladen Dimitrov for his interest in my approach to Hilbert modular symbols. Most of all I would like to thank to Yuri Manin for his inspiring talk at the Max Planck Institute for Mathematics in Bonn on noncommutative modular symbols and for his encouragement and interest in the work presented here. Finally, I would like to thank the referees for the useful suggestions and commentaries.

I would like to thank the Max Planck Institute for Mathematics in Bonn and the Marie Curie Research Training Network, Arithmetic Algebraic Geometry Network at Durham University for financial support in 2004-2005 and 2005-2006, respectively. 


\section{References}

[Ash and Borel 1990] A. Ash and A. Borel, "Generalized modular symbols", pp. 57-75 in Cohomology of arithmetic groups and automorphic forms (Luminy-Marseille, 1989), edited by J.-P. Labesse and J. Schwermer, Lecture Notes in Math. 1447, Springer, Berlin, 1990. MR 92e:11058 Zbl 0719.11033

[Ash and Rudolph 1979] A. Ash and L. Rudolph, "The modular symbol and continued fractions in higher dimensions”, Invent. Math. 55:3 (1979), 241-250. MR 82g:12011 Zbl 0426.10023

[Berger et al. 2013] L. Berger, G. Böckle, L. Dembélé, M. Dimitrov, T. Dokchitser, and J. Voight, Elliptic curves, Hilbert modular forms and Galois deformations, Birkhäuser/Springer, Basel, 2013. MR 3155300 Zbl 1276.11004

[Birch 1971] B. J. Birch, "Elliptic curves over Q: a progress report", pp. 396-400 in 1969 Number Theory Institute (Stony Brook, 1969), edited by D. J. Lewis, Proc. Symp. Pure Math. 20, Amer. Math. Soc., Providence, R.I., 1971. MR 47 \#3395 Zbl 0214.19801

[Borceux 1994] F. Borceux, Handbook of categorical algebra I: Basic category theory, Encyclopedia of Mathematics and its Applications 50, Cambridge University Press, 1994. MR 96g:18001a Zbl 0803.18001

[Borel and Serre 1973] A. Borel and J.-P. Serre, "Corners and arithmetic groups", Comment. Math. Helv. 48 (1973), 436-491. MR 52 \#8337 Zbl 0274.22011

[Brown 1982] K. S. Brown, Cohomology of groups, Graduate Texts in Mathematics 87, Springer, Berlin, 1982. MR 83k:20002 Zbl 0584.20036

[Bruinier et al. 2008] J. H. Bruinier, G. van der Geer, G. Harder, and D. Zagier, The 1-2-3 of modular forms: lectures at a summer school in Nordfjordeid, Norway, Springer, Berlin, 2008. MR 2009d:11002 Zbl 1197.11047

[Chen 1977] K. T. Chen, "Iterated path integrals”, Bull. Amer. Math. Soc. 83:5 (1977), 831-879. MR 56 \#13210 Zbl 0389.58001

[Cremona 1997] J. E. Cremona, Algorithms for modular elliptic curves, 2nd ed., Cambridge University Press, 1997. MR 99e:11068 Zbl 0872.14041

[Drinfeld 1973] V. G. Drinfeld, “Two theorems on modular curves”, Funkcional. Anal. i Prilozhen. 7:2 (1973), 83-84. In Russian; translated in Functional Anal. Appl. 7:2 (1973), 155-156. MR 47 \#6705 Zbl 0285.14006

[Euler 1748] L. Euler, Introductio in analysin infinitorum, I, 1748. Translated as Introduction to analysis of the infinite, Book I, Springer, New York, 1988. MR 89g:01067 Zbl 0657.01013

[Freitag 1990] E. Freitag, Hilbert modular forms, Springer, Berlin, 1990. MR 91c:11025 Zbl 0702. 11029

[Gangl et al. 2006] H. Gangl, M. Kaneko, and D. Zagier, "Double zeta values and modular forms", pp. 71-106 in Automorphic forms and zeta functions, edited by S. Böcherer et al., World Sci. Publ., Hackensack, NJ, 2006. MR 2006m:11138 Zbl 1122.11057

[Goncharov 2001a] A. B. Goncharov, "Multiple polylogarithms and mixed Tate motives", preprint, 2001. arXiv math/0103059

[Goncharov 2001b] A. B. Goncharov, "Multiple $\zeta$-values, Galois groups, and geometry of modular varieties", pp. 361-392 in European Congress of Mathematics (Barcelona, 2000), vol. I, edited by C. Casacuberta et al., Progr. Math. 201, Birkhäuser, Basel, 2001. MR 2003g:11073 Zbl 1042.11042

[Goncharov 2001c] A. B. Goncharov, "The dihedral Lie algebras and Galois symmetries of $\pi_{1}^{(l)}\left(\mathbb{P}^{1}\right.$ $\left.\left(\{0, \infty\} \cup \mu_{N}\right)\right) ”$, Duke Math. J. 110:3 (2001), 397-487. MR 2002m:14020 Zbl 1113.14020 
[Gunnells 2000a] P. E. Gunnells, "Finiteness of minimal modular symbols for SL $n$ ", J. Number Theory 82:1 (2000), 134-139. MR 2001e:11051 Zbl 0987.11040

[Gunnells 2000b] P. E. Gunnells, "Symplectic modular symbols", Duke Math. J. 102:2 (2000), 329-350. MR 2001i:11062 Zbl 0988.11023

[Gunnells and Yasaki 2008] P. E. Gunnells and D. Yasaki, "Hecke operators and Hilbert modular forms", pp. 387-401 in Algorithmic number theory, edited by A. J. van der Poorten and A. Stein, Lecture Notes in Comput. Sci. 5011, Springer, Berlin, 2008. MR 2010a:11081 Zbl 1205.11056

[Hartshorne 1977] R. Hartshorne, Algebraic geometry, Graduate Texts in Mathematics 52, Springer, New York, 1977. MR 57 \#3116 Zbl 0367.14001

[Hirzebruch 1973] F. E. P. Hirzebruch, "Hilbert modular surfaces", Enseign. Math. (2) 19 (1973), 183-281. MR 52 \#10750 Zbl 0285.14007

[Hirzebruch and Zagier 1976] F. Hirzebruch and D. Zagier, "Intersection numbers of curves on Hilbert modular surfaces and modular forms of Nebentypus”, Invent. Math. 36 (1976), 57-113. MR 56 \#11909 Zbl 0332.14009

[Horozov 2006] I. Horozov, "Non-commutative two dimensional modular symbol”, preprint, 2006. arXiv math/0611955

[Horozov 2011] I. Horozov, "Non-abelian reciprocity laws on a Riemann surface", Int. Math. Res. Not. 2011:11 (2011), 2469-2495. MR 2012d:19006 Zbl 1218.30127

[Horozov 2014a] I. Horozov, "Cohomology of GL(4, ZZ) with nontrivial coefficients", Math. Res. Lett. 21:5 (2014), 1111-1136.

[Horozov 2014b] I. Horozov, "Multiple Dedekind zeta functions", J. Reine Angew. Math. (online publication July 2014).

[Horozov 2014c] I. Horozov, "Reciprocity laws on algebraic surfaces via iterated integrals", $J$. K-Theory 14:2 (2014), 273-312. MR 3264264 Zbl 06369821

[Kazhdan et al. 2000] D. Kazhdan, B. Mazur, and C.-G. Schmidt, "Relative modular symbols and Rankin-Selberg convolutions”, J. Reine Angew. Math. 519 (2000), 97-141. MR 2001j:11026 Zbl 0941.11020

[Kontsevich and Zagier 2001] M. Kontsevich and D. Zagier, "Periods", pp. 771-808 in Mathematics unlimited -2001 and beyond, edited by B. Engquist and W. Schmid, Springer, Berlin, 2001. MR 2002i:11002 Zbl 1039.11002

[Lurie 2009] J. Lurie, Higher topos theory, Annals of Mathematics Studies 170, Princeton University Press, NJ, 2009. MR 2010j:18001 Zbl 1175.18001

[Manin 1972] J. I. Manin, "Parabolic points and zeta functions of modular curves", Izv. Akad. Nauk SSSR Ser. Mat. 36 (1972), 19-66. In Russian; translated in Math. USSR Izv. 6:1 (1972), 19-64. MR 47 \#3396 Zbl 0243.14008

[Manin 2006] Y. I. Manin, "Iterated integrals of modular forms and noncommutative modular symbols", pp. 565-597 in Algebraic geometry and number theory, edited by V. Ginzburg, Progr. Math. 253, Birkhäuser, Boston, 2006. MR 2008a:11062 Zbl 1184.11019

[Manin 2009] Y. I. Manin, "Lectures on modular symbols", pp. 137-152 in Arithmetic geometry, edited by H. Darmon et al., Clay Math. Proc. 8, Amer. Math. Soc., Providence, RI, 2009. MR 2010i:11068 Zbl 1222.11066

[Mazur 1973] B. Mazur, "Courbes elliptiques et symboles modulaires”, exposeé no. 414, 277-294 in Séminaire Bourbaki, 1971/1972, Lecture Notes in Math. 317, Springer, Berlin, 1973. MR 55 \#2930 Zbl 0276.14012 
[Mazur and Swinnerton-Dyer 1974] B. Mazur and P. Swinnerton-Dyer, "Arithmetic of Weil curves", Invent. Math. 25 (1974), 1-61. MR 50 \#7152 Zbl 0281.14016

[Parshin 1966] A. N. Parshin, "On a certain generalization of Jacobian manifold", Izv. Akad. Nauk SSSR Ser. Mat. 30 (1966), 175-182. In Russian. MR 33 \#4956 Zbl 0163.15303

[Shimura 1978] G. Shimura, "The special values of the zeta functions associated with Hilbert modular forms”, Duke Math. J. 45:3 (1978), 637-679. MR 80a:10043 Zbl 0394.10015

[Shintani 1976] T. Shintani, "On evaluation of zeta functions of totally real algebraic number fields at non-positive integers”, J. Fac. Sci. Univ. Tokyo Sect. IA Math. 23:2 (1976), 393-417. MR 55 \#266 Zbl 0349.12007

[Shokurov 1980] V. V. Shokurov, "Shimura integrals of cusp forms", Izv. Akad. Nauk SSSR Ser. Mat. 44:3 (1980), 670-718. In Russian; translated in Math. USSR Izv. 16:3 (1981), 603-646. MR 82b:10029 Zbl 0444.14030

[Stein 2007] W. Stein, Modular forms, a computational approach, Graduate Studies in Mathematics 79, Amer. Math. Soc., Providence, RI, 2007. MR 2008d:11037 Zbl 1110.11015

[Street 1982] R. Street, “Two-dimensional sheaf theory", J. Pure Appl. Algebra 23:3 (1982), 251-270. MR 83d:18014 Zbl 0478.18004

[Zagier 1976] D. Zagier, "On the values at negative integers of the zeta-function of a real quadratic field”, Enseignement Math. (2) 22:1-2 (1976), 55-95. MR 53 \#10742 Zbl 0334.12021

Communicated by Yuri Manin

Received 2013-08-22 Revised 2014-09-17 Accepted 2014-11-26

horozov@math.wustl.edu Department of Mathematics,

Washington University in St. Louis, One Brookings Drive, Campus Box 1146, Saint Louis, MO 63130, United States 


\section{Algebra \& Number Theory}

msp.org/ant

\section{EDITORS}

MANAGING EDITOR

Bjorn Poonen

Massachusetts Institute of Technology

Cambridge, USA

\author{
EDITORIAL BOARD CHAIR \\ David Eisenbud \\ University of California \\ Berkeley, USA
}

BOARD OF EDITORS

Georgia Benkart

Dave Benson

Richard E. Borcherds

John H. Coates

J-L. Colliot-Thélène

Brian D. Conrad

Hélène Esnault

Hubert Flenner

Edward Frenkel

Andrew Granville

Joseph Gubeladze

Roger Heath-Brown

Craig Huneke

János Kollár

Yuri Manin

Barry Mazur

Philippe Michel

Susan Montgomery
University of Wisconsin, Madison, USA

University of Aberdeen, Scotland

University of California, Berkeley, USA

University of Cambridge, UK

CNRS, Université Paris-Sud, France

University of Michigan, USA

Freie Universität Berlin, Germany

Ruhr-Universität, Germany

University of California, Berkeley, USA

Université de Montréal, Canada

San Francisco State University, USA

Oxford University, UK

University of Virginia, USA

Princeton University, USA

Northwestern University, USA

Harvard University, USA

École Polytechnique Fédérale de Lausanne

University of Southern California, USA
Shigefumi Mori

Raman Parimala

Jonathan Pila

Anand Pillay

Victor Reiner

Peter Sarnak

Joseph H. Silverman

Michael Singer

Vasudevan Srinivas

J. Toby Stafford

Richard Taylor

Ravi Vakil

Michel van den Bergh

Marie-France Vignéras

Kei-Ichi Watanabe

Efim Zelmanov

Shou-Wu Zhang
RIMS, Kyoto University, Japan

Emory University, USA

University of Oxford, UK

University of Notre Dame, USA

University of Minnesota, USA

Princeton University, USA

Brown University, USA

North Carolina State University, USA

Tata Inst. of Fund. Research, India

University of Michigan, USA

Harvard University, USA

Stanford University, USA

Hasselt University, Belgium

Université Paris VII, France

Nihon University, Japan

University of California, San Diego, USA

Princeton University, USA

\section{PRODUCTION}

production@msp.org

Silvio Levy, Scientific Editor

See inside back cover or msp.org/ant for submission instructions.

The subscription price for 2015 is US \$255/year for the electronic version, and $\$ 440 /$ year ( $\$ 55$, if shipping outside the US) for print and electronic. Subscriptions, requests for back issues and changes of subscribers address should be sent to MSP.

Algebra \& Number Theory (ISSN 1944-7833 electronic, 1937-0652 printed) at Mathematical Sciences Publishers, 798 Evans Hall \#3840, c/o University of California, Berkeley, CA 94720-3840 is published continuously online. Periodical rate postage paid at Berkeley, CA 94704, and additional mailing offices.

ANT peer review and production are managed by EditFLOW ${ }^{\circledR}$ from MSP.

\section{PUBLISHED BY}

7 mathematical sciences publishers

nonprofit scientific publishing

http://msp.org/

(C) 2015 Mathematical Sciences Publishers 


\section{Algebra \& Number Theory}

Volume $9 \quad$ No. $2 \quad 2015$

Lifting harmonic morphisms II: Tropical curves and metrized complexes

OMID AMINI, MATTHEW BAKER, ERWAN BRUgallé and JosePH RABINOFF

Noncommutative Hilbert modular symbols

IVAN HOROZOV

$p$-adic Hodge theory in rigid analytic families

REBECCA BELLOVIN

Semistable periods of finite slope families

RUOCHUAN LIU

ANAND DEOPURKAR and ANAND PATEL

Finite-dimensional quotients of Hecke algebras

493

IVAN LOSEV

Semiample invertible sheaves with semipositive continuous hermitian metrics

503 ATSUSHI MORIWAKI 